Ann. Scient. Éc. Norm. Sup.,

$4^{\text {e }}$ série, t. 39, 2006, p. 245 à 300.

\title{
CRYSTALLINE BOUNDEDNESS PRINCIPLE
}

\author{
BY ADRIAN VASIU
}

ABSTRACT. - We prove that an $F$-crystal $(M, \varphi)$ over an algebraically closed field $k$ of characteristic $p>0$ is determined by $(M, \varphi) \bmod p^{n}$, where $n \geqslant 1$ depends only on the rank of $M$ and on the greatest Hodge slope of $(M, \varphi)$. We also extend this result to triples $(M, \varphi, G)$, where $G$ is a flat, closed subgroup scheme of $\mathbf{G L}_{M}$ whose generic fibre is connected and has a Lie algebra normalized by $\varphi$. We get two purity results. If $\mathfrak{C}$ is an $F$-crystal over a reduced $\mathbf{F}_{p}$-scheme $S$, then each stratum of the Newton polygon stratification of $S$ defined by $\mathfrak{C}$, is an affine $S$-scheme (a weaker result was known before for $S$ noetherian). The locally closed subscheme of the Mumford scheme $\mathcal{A}_{d, 1, N_{k}}$ defined by the isomorphism class of a principally quasi-polarized $p$-divisible group over $k$ of height $2 d$, is an affine $\mathcal{A}_{d, 1, N_{k}}$-scheme.

(c) 2006 Elsevier SAS

RÉSUMÉ. - Nous prouvons qu'un $F$-cristal $(M, \varphi)$ défini sur un corps $k$ algébriquement clos de caractéristique $p>0$ est déterminé par $(M, \varphi) \bmod p^{n}$, où $n \geqslant 1$ dépend seulement du rang de $M$ et de la plus grande pente de Hodge de $(M, \varphi)$. On étend ce résultat aux triplets $(M, \varphi, G)$, où $G$ est un sous-groupe fermé et plat de $\mathbf{G L}_{M}$ dont la fibre générique est connexe et a une algèbre de Lie normalisée par $\varphi$. Nous obtenons deux résultats de pureté. Si $\mathfrak{C}$ est un $F$-cristal sur un $\mathbf{F}_{p}$-schéma réduit $S$, alors chaque strate de la stratification du polygone de Newton de $S$ défini par $\mathfrak{C}$ est un $S$-schéma affine (un résultat moins général était déjà connu pour $S$ noethérien). Le sous-schéma localement fermé du schéma de Mumford $\mathcal{A}_{d, 1, N_{k}}$ défini par la classe d'isomorphisme d'un groupe $p$-divisible principalement quasi polarisé sur $k$ de hauteur $2 d$ est un $\mathcal{A}_{d, 1, N_{k}}$-schéma affine.

(c) 2006 Elsevier SAS

\section{Introduction}

Let $p \in \mathbf{N}$ be a prime. Let $k$ be a perfect field of characteristic $p$. Let $\bar{k}$ be an algebraic closure of $k$. Let $W(k)$ be the Witt ring of $k$. Let $B(k):=W(k)\left[\frac{1}{p}\right]$ be the field of fractions of $W(k)$. Let $\sigma:=\sigma_{k}$ be the Frobenius automorphism of $k, W(k)$, and $B(k)$. A group scheme $H$ over $\operatorname{Spec}(W(k))$ is called integral if $H$ is flat over $\operatorname{Spec}(W(k))$ and $H_{B(k)}$ is connected (i.e. if the scheme $H$ is integral). Let $\operatorname{Lie}\left(H_{B(k)}\right)$ be the Lie algebra over $B(k)$ of $H_{B(k)}$. If $H$ is smooth over $\operatorname{Spec}(W(k))$, let $\operatorname{Lie}(H)$ be the Lie algebra over $W(k)$ of $H$. If $O$ is a free module of finite rank over some commutative $\mathbf{Z}$-algebra $R$, let $\mathbf{G L}_{O}$ be the group scheme over $\operatorname{Spec}(R)$ of linear automorphisms of $O$.

Let $(r, d) \in \mathbf{N} \times(\mathbf{N} \cup\{0\})$, with $r \geqslant d$. Let $D$ be a $p$-divisible group over $\operatorname{Spec}(\bar{k})$ of height $r$ and dimension $d$. It is well known that if $d \in\{0,1, r-1, r\}$, then:

(*) $D$ is uniquely determined up to isomorphism by its p-torsion subgroup scheme $D[p]$.

But $(*)$ does not hold if $2 \leqslant d \leqslant r-2$. In 1963 Manin published an analogue of $(*)$ for $2 \leqslant d \leqslant r-2$ but unfortunately he separated it into three parts (see [28, p. 44, 3.6, and 3.8] and below). Only recently, this paper and [36] contain explicit analogues of $(*)$ for $2 \leqslant d \leqslant r-2$. The two main reasons for this delay in the literature are: (i) the widely spread opinion, which 
goes back more than 40 years, that $p$-divisible groups involve an infinite process, and (ii) the classification results of [28, p. 44] were rarely used. Our point of view is that $F$-crystals in locally free sheaves of finite rank over many $\operatorname{Spec}(k)$-schemes $Y$ involve a bounded infinite process. In this paper we give meaning to this point of view for the case $Y=\operatorname{Spec}(\bar{k})$. We start with few definitions.

\subsection{Definitions. -}

(a) By a latticed F-isocrystal with a group over $k$ we mean a triple $(M, \varphi, G)$, where $M$ is a free $W(k)$-module of finite rank, where $\varphi$ is a $\sigma$-linear automorphism of $M\left[\frac{1}{p}\right]$, and where $G$ is an integral, closed subgroup scheme of $\mathbf{G L}_{M}$, such that the Lie subalgebra $\operatorname{Lie}\left(G_{B(k)}\right)$ of $\operatorname{End}\left(M\left[\frac{1}{p}\right]\right)$ is normalized by $\varphi$. Here we denote also by $\varphi$ the $\sigma$-linear (algebra) automorphism of $\operatorname{End}\left(M\left[\frac{1}{p}\right]\right)$ that takes $e \in \operatorname{End}\left(M\left[\frac{1}{p}\right]\right)$ into $\varphi \circ e \circ \varphi^{-1} \in \operatorname{End}\left(M\left[\frac{1}{p}\right]\right)$. If $G=\mathbf{G L}_{M}$, then often we do not mention $G$ and we omit "with a group".

(b) By an isomorphism between two latticed $F$-isocrystals with a group $\left(M_{1}, \varphi_{1}, G_{1}\right)$ and $\left(M_{2}, \varphi_{2}, G_{2}\right)$ over $k$ we mean a $W(k)$-linear isomorphism $f: M_{1} \stackrel{\sim}{\longrightarrow} M_{2}$ such that $\varphi_{2} \circ f=$ $f \circ \varphi_{1}$ and the isomorphism $\mathbf{G L}_{M_{1}} \stackrel{\sim}{\longrightarrow} \mathbf{G L}_{M_{2}}$ induced by $f$, takes $G_{1}$ onto $G_{2}$.

The pair $\left(M\left[\frac{1}{p}\right], \varphi\right)$ is called an $F$-isocrystal over $k$. If we have $p M \subseteq \varphi(M) \subseteq M$, then the pair $(M, \varphi)$ is called a Dieudonné module over $k$. For $g^{\prime} \in G(B(k))$ let $g^{\prime} \varphi$ be the $\sigma$-linear automorphism of $M\left[\frac{1}{p}\right]$ that takes $x \in M\left[\frac{1}{p}\right]$ into $g^{\prime}(\varphi(x)) \in M\left[\frac{1}{p}\right]$. The triple $\left(M, g^{\prime} \varphi, G\right)$ is also a latticed $F$-isocrystal with a group over $k$.

Often there exists a "good" class $\mathbf{M}$ of motives over $k$ that has the following property. The crystalline realization of any motive $\mathcal{M}$ in $\mathbf{M}$ is naturally identified with $\left(M, g_{\mathcal{M}} \varphi\right)$ for some $g_{\mathcal{M}} \in G(W(k))$ and moreover $G_{B(k)}$ is the identity component of the subgroup of $\mathbf{G L}_{M\left[\frac{1}{p}\right]}$ that fixes some tensors of the tensor algebra of $M\left[\frac{1}{p}\right] \oplus \operatorname{Hom}\left(M\left[\frac{1}{p}\right], B(k)\right)$ which do not depend on $\mathcal{M}$ and which are (expected to be) crystalline realizations of motives over $k$ that are intrinsically associated to $\mathcal{M}$. For instance, see [40, 5 and $\S 6]$ for contexts that pertain to classes of $H^{1}$ motives of abelian varieties over $\operatorname{Spec}(k)$ which are associated to $k$-valued points of a (fixed) good integral model of a Shimura variety of Hodge type. The paper [40] and many previous ones (like [25]) deal with particular cases of such triples $(M, \varphi, G)$ 's: the pair $(M, \varphi)$ is a Dieudonné module over $k$, the group scheme $G$ is reductive, and there exists a semisimple element $s_{\varphi} \in G(B(k))$ whose eigenvalues are 1 and $p$ and such that $\varphi s_{\varphi}^{-1}$ is a $\sigma$-linear automorphism of $M$. Any good classification of the triples $\left(M, g_{\mathcal{M}} \varphi, G\right)$ up to isomorphisms defined by elements of $G(W(k))$, is often an important tool toward the classification of motives in $\mathrm{M}$.

Classically, one approaches the classification of all triples $(M, g \varphi, G)$ with $g \in G(W(k))$, up to isomorphisms defined by elements of $G(W(k))$, in two steps. The first step aims to classify $\left(M\left[\frac{1}{p}\right], g \varphi, G_{B(k)}\right)$ 's up to isomorphisms defined by elements of $G(B(k))$. The second step aims to use the first step in order to study $(M, g \varphi, G)$ 's.

A systematic and general approach to the first step was started in [24], which works in the context in which the group $G_{B(k)}$ is reductive, $k=\bar{k}$, and the pair $\left(M\left[\frac{1}{p}\right], G_{B(k)}\right)$ has a $\mathbf{Q}_{p}$ structure $\left(M_{\mathbf{Q}_{p}}, G_{\mathbf{Q}_{p}}\right)$ with respect to which $\varphi$ becomes $g_{\varphi}\left(1_{M_{\mathbf{Q}_{p}}} \otimes \sigma\right)$ for some $g_{\varphi} \in G(B(k))$; thus, in order to classify $\left(M\left[\frac{1}{p}\right], g \varphi, G_{B(k)}\right)$ 's up to isomorphisms defined by elements of $G(B(k))$, one only has to describe the image $G_{\varphi}$ of the set $\left\{g g_{\varphi} \mid g \in G(W(k))\right\}$ in the set $B\left(G_{\mathbf{Q}_{p}}\right)$ of $\sigma$-conjugacy classes of elements of $G_{\mathbf{Q}_{p}}(B(k))=G(B(k))$. Even if $k=\bar{k}$, in general such $\mathbf{Q}_{p}$ structures do not exist (for instance, they do not exist if the group $G_{B(k)}$ is commutative and $\left(\operatorname{Lie}\left(G_{B(k)}\right), \varphi\right)$ has non-zero slopes).

One can define two natural equivalence relations $I_{\varphi}$ and $R_{\varphi}$ on the set underlying the group $G(W(k))$ as follows. A pair $\left(g_{1}, g_{2}\right) \in G(W(k))^{2}$ belongs to $I_{\varphi}$ (respectively to $R_{\varphi}$ ) if and only

$4^{\text {e }}$ SÉRIE - TOME $39-2006-\mathrm{N}^{\circ} 2$ 
if there exists $g_{12} \in G(W(k))$ (respectively $g_{12} \in G(B(k))$ ) such that $g_{12} g_{1} \varphi=g_{2} \varphi g_{12}$. The set of isomorphism classes of $(M, g \varphi, G)$ 's (up to isomorphisms defined by elements of $G(W(k))$ ) is in natural bijection to the quotient set $G(W(k)) / I_{\varphi}$. The quotient set $G(W(k)) / R_{\varphi}$ is a more general version of the above type of sets $G_{\varphi}$. In general, the natural surjective map $G(W(k)) / I_{\varphi} \rightarrow G(W(k)) / R_{\varphi}$ is not an injection and some of its fibres have the same cardinality as $k$. In general, one can not "recover" $(M, g \varphi, G)$ and its reductions modulo powers of $p$ from the equivalence class $[g] \in G(W(k)) / I_{\varphi}$ and from the triple $\left(M\left[\frac{1}{p}\right], g \varphi, G_{B(k)}\right)$. The last two sentences explain why in this paper, for the study of the quotient set $G(W(k)) / I_{\varphi}$ and of (reductions modulo powers of $p$ of) $(M, g \varphi, G)$ 's, we can not appeal to the results of [24,37], etc. In addition, the language of latticed $F$-isocrystals is more general and more suited for reductions modulo powers of $p$, for endomorphisms, for deformations, and for functorial purposes than the language of either $\sigma$-conjugacy classes or equivalence classes of $I_{\varphi}$.

If $g_{1}, g_{2}, g_{12} \in G(W(k))$ satisfy $g_{12} g_{1} \varphi=g_{2} \varphi g_{12}$, it is of interest to keep track of the greatest number $n_{12} \in \mathbf{N} \cup\{0\}$ such that $g_{12}$ and $1_{M}$ are congruent $\bmod p^{n_{12}}$. As the relation $I_{\varphi}$ is not suitable for this purpose, it will not be used outside this introduction.

The set $\{(M, g \varphi, G) \mid g \in G(W(k))\}$ is in natural bijection to $G(W(k))$. Any set of the form $\{(M, g \varphi, G) \mid g \in G(W(k))\}$ will be called a family of latticed $F$-isocrystals with a group over $k$. This paper is a starting point for general classifications of families of latticed $F$-isocrystals with a group over $\bar{k}$. The fact that such classifications are achievable is supported by the following universal principle.

1.2. MAin Theorem A (Crystalline Boundedness Principle).-Suppose $k=\bar{k}$. Let $(M, \varphi, G)$ be a latticed F-isocrystal with a group over $k$. Then there exists a number $n_{\text {fam }} \in \mathbf{N} \cup\{0\}$ that is effectively bounded from above and that has the property that for any pair $\left(g, g_{n_{\mathrm{fam}}}\right) \in G(W(k))^{2}$ such that $g_{n_{\mathrm{fam}}}$ is congruent $\bmod p^{n_{\mathrm{fam}}}$ to $1_{M}$, there exist isomorphisms between $(M, g \varphi, G)$ and $\left(M, g_{n_{\mathrm{fam}}} g \varphi, G\right)$ which are elements of $G(W(k))$.

Thus the equivalence class $[g] \in G(W(k)) / I_{\varphi}$ depends only on $g$ mod $p^{n_{\text {fam }}}$; this supports our bounded infinite process point of view. If $G=\mathbf{G L}_{M}$ and $(M, \varphi)$ is a Dieudonné module over $k$, then Main Theorem A is a direct consequence of [28, p. 44, 3.6, and 3.8]. By a classical theorem of Dieudonné (see [7, Thms. 3 and 5], [28, §2], [5, Ch. IV, §4], or [14, Ch. III, §6]), the category of $p$-divisible groups over $\operatorname{Spec}(k)$ is anti-equivalent to the category of Dieudonné modules over $k$. Thus we get a new proof of the following result which in essence is due to Manin and which is also contained in [36].

1.3. Corollary. - There exists a smallest number $T(r, d) \in \mathbf{N} \cup\{0\}$ such that any p-divisible group $D$ over $\operatorname{Spec}(\bar{k})$ of height $r$ and dimension $d$, is uniquely determined up to isomorphism by its $p^{T(r, d)}$-torsion subgroup scheme $D\left[p^{T(r, d)}\right]$. Upper bounds of $T(r, d)$ are effectively computable in terms of $r$.

\subsection{On the proof of Main Theorem A}

The proof of Main Theorem A (see 3.1) relies on what we call the stairs method. The method is rooted on the simple fact that for any $t \in \mathbf{N}$ and every $y, z \in \operatorname{End}(M)$, the two automorphisms $1_{M}+p^{t} y$ and $1_{M}+p^{t} z$ of $M$ commute $\bmod p^{2 t}$. To outline the method, we assume in this paragraph that $G$ is smooth over $\operatorname{Spec}(W(k))$. Let $m \in \mathbf{N} \cup\{0\}$ be the smallest number for which there exists a $W(k)$-submodule $E$ of $\operatorname{Lie}(G)$ that contains $p^{m}(\operatorname{Lie}(G))$ and that has a $W(k)$-basis $\left\{e_{1}, e_{2}, \ldots, e_{v}\right\}$ such that for $l \in\{1, \ldots, v\}$ we have $\varphi\left(e_{l}\right)=p^{n_{l}} e_{\pi(l)}$, where $\pi$ is a permutation of the set $\{1, \ldots, v\}$ and where $n_{l}$ 's are integers that have the following stairs property. For any cycle $\left(l_{1}, \ldots, l_{q}\right)$ of $\pi$, the integers $n_{l_{1}}, \ldots, n_{l_{q}}$ are either all non-negative or all non-positive. The existence of $m$ is implied by Dieudonné's classification of $F$-isocrystals 
over $k$ (see [28, §2]). In general, the $W(k)$-submodule $E$ is not a Lie subalgebra of $\operatorname{Lie}(G)$. For any $\tilde{g} \in G(W(k))$ congruent $\bmod p^{2 m+t}$ to $1_{M}$, there exists $\tilde{e} \in E$ such that the elements $\tilde{g}$ and $1_{M}+p^{m+t} \tilde{e}$ of $\mathbf{G L}_{M}(W(k))$ are congruent $\bmod p^{2 m+1+t}$. Due to this and the stairs property, for $p \geqslant 3$ there exists an isomorphism between $(M, \tilde{g} \varphi, G)$ and $(M, \varphi, G)$ which is an element $\tilde{g}_{0} \in G(W(k))$ congruent $\bmod p^{m+t}$ to $1_{M}$ (see 3.1.1). If $p=2$, then a slight variant of this holds. Exponential maps (see 2.6) substitute from many points of view the classical Verschiebung maps of Dieudonné modules; for instance, one can choose $\tilde{g}_{0}$ to be an infinite product of exponential elements of the form $\sum_{i=0}^{\infty} \frac{p^{i(m+1)}}{i !} e^{i}$, where $e \in E$. See 2.2 to 2.4 for the $\sigma$-linear preliminaries that are necessary for the estimates which give us the effectiveness part of Main Theorem A. These estimates provide inductively upper bounds of $m$ in terms of $\operatorname{dim}\left(G_{B(k)}\right)$ and of the $s$-number and the $h$-number of the latticed $F$-isocrystal $(\operatorname{Lie}(G), \varphi)$ over $k$ (see 2.2.1(e) for these two non-negative integers which do not change if $\varphi$ is replaced by $g \varphi$ ).

\subsection{Complements, examples, and applications}

See 3.2 for interpretations and variants of Main Theorem A in terms of reductions modulo powers of $p$; in particular, see 3.2.4 for the passage from Main Theorem A to Corollary 1.3. In 3.3 we improve (in many cases of interest) the upper bounds (of $n_{\mathrm{fam}}$, etc.) we obtain in 3.1.1 to 3.1 .5 .

In Section 4 we include four examples. It is well known that if the $p$-divisible group $D$ is ordinary, then $D$ is uniquely determined up to isomorphism by $D[p]$ and moreover $D$ has a unique lift to $\operatorname{Spec}(W(\bar{k}))$ (called the canonical lift) that has the property that any endomorphism of $D$ lifts to it. Example 2 identifies the type of latticed $F$-isocrystals with a group over $\bar{k}$ to which the last two facts generalize naturally (see 4.3.1 and 4.3.2). Example 4 shows that if $r=2 d$, $d \geqslant 3$, and the slopes of the Newton polygon of $D$ are $\frac{1}{d}$ and $\frac{d-1}{d}$, then $D$ is uniquely determined up to isomorphism by $D\left[p^{3}\right]$ (see 4.5 ).

In Section 5 we list four direct applications of Main Theorem A and of 3.2. First we present the homomorphism form of Main Theorem A (see 5.1.1). Second we define transcendental degrees of definition for many classes of latticed $F$-isocrystals with a group over $\bar{k}$ (see 5.2). When the transcendental degrees of definition are 0 , we also define (finite) fields of definition. In particular, Theorem 5.2.3 (when combined with Lemma 3.2.2) implies that it is possible to build up an atlas and a list of tables of isomorphism classes of $p$-divisible groups (endowed with certain extra structures) over $\operatorname{Spec}(\bar{k})$ that are definable over the spectrum of a fixed finite field $\mathbf{F}_{p^{q}}$, which are similar in nature to the atlas of finite groups (see [3]) and to the list of tables of elliptic curves over $\operatorname{Spec}(\mathbf{Q})$ (see [4]).

Let $N \in \mathbf{N} \backslash\{1,2\}$ be relatively prime to $p$. Let $\mathcal{A}_{d, 1, N}$ be the smooth, quasi-projective Mumford moduli scheme over $\operatorname{Spec}\left(\mathbf{F}_{p}\right)$ that parametrizes isomorphism classes of principally polarized abelian schemes with level- $N$ structure and of relative dimension $d$ over $\operatorname{Spec}\left(\mathbf{F}_{p}\right)$ schemes (see [33, Thms. 7.9 and 7.10]). Third we apply the principally quasi-polarized version of Corollary 1.3 (see 3.2.5) to get a new type of stratification of $\mathcal{A}_{d, 1, N}$. Here the word stratification is used in a wide sense (see 2.1.1) which allows the number of strata to be infinite. The strata we get are defined by isomorphism classes of principally quasi-polarized $p$-divisible groups of height $2 d$ over spectra of algebraically closed fields of characteristic $p$; they are regular and equidimensional (see 5.3.1 and 5.3.2). Moreover, this new type of stratification of $\mathcal{A}_{d, 1, N}$ satisfies the purity property we define in 2.1.1, i.e. its strata are affine $\mathcal{A}_{d, 1, N}$-schemes (see 5.3.1 and 5.3.2). Variants of 1.3, 3.2.5, 3.2.6, and 5.3.2 but without its purity property part, are also contained in [36].

Fourth we get a new proof (see 5.4) of the "Katz open part" of the Grothendieck-Katz specialization theorem for Newton polygons (see [22, 2.3.1 and 2.3.2]).

4e SÉRIE - TOME $39-2006-\mathrm{N}^{\circ} 2$ 
The main goal of Section 6 is to prove the following result (see 6.1 and 6.2).

1.6. MAIN THEOREM B. - Let $\mathfrak{C}$ be an F-crystal in locally free sheaves of finite rank over a reduced $\operatorname{Spec}\left(\mathbf{F}_{p}\right)$-scheme $S$. Then the Newton polygon stratification of $S$ defined by $\mathfrak{C}$ satisfies the purity property (i.e. each stratum of it is an affine $S$-scheme).

A variant of Main Theorem B was obtained first in [10, 4.1], for the particular case when $S$ is locally noetherian. The fact that the variant is a weaker form of Main Theorem B is explained in 6.3(a). The main new idea of Section 6 is: Newton polygons are encoded in the existence of suitable morphisms between different evaluations of $F$-crystals (viewed without connections) at Witt schemes of (effectively computable) finite lengths. The proof of Main Theorem B combines this new idea with the results of Katz (see [22, 2.6 and 2.7]) on isogenies between $F$-crystals of constant Newton polygons over spectra of (perfections of) complete, discrete valuation rings that are of the form $\bar{k}[[x]]$.

\section{Preliminaries}

See 2.1 for our main notations and conventions. See 2.2 for few definitions and simple properties that pertain to latticed $F$-isocrystals with a group over $k$. In particular, in 2.2.2 we define Dieudonné-Fontaine torsions and volumes of latticed $F$-isocrystals. Inequalities and estimates on such torsions are gathered in 2.3 and 2.4 (respectively); they are essential for examples and for the effectiveness part of 1.2. In 2.5 we apply [42] to get $\mathbf{Z}_{p}$ structures for many classes of latticed $F$-isocrystals with a group over $\bar{k}$. In 2.6 and 2.7 we include group scheme theoretical properties that are needed in Sections 3 and 4. In 2.8 we present complements on the categories $\mathcal{M}\left(W_{q}(S)\right)$ we will introduce in 2.1. In 2.9 we recall two results of commutative algebra. Sections 2.8 and 2.9 are not used before 5.4. For Newton polygons of $F$-isocrystals over $k$ we refer to $[22,1.3]$.

\subsection{Notations and conventions}

By $w$ we denote an arbitrary variable. If $q \in \mathbf{N}$, let $\mathbf{F}_{p^{q}}$ be the field with $p^{q}$ elements. If $R$ is a commutative $\mathbf{F}_{p}$-algebra, let $W(R)$ be the Witt ring of $R$ and let $W_{q}(R)$ be the ring of Witt vectors of length $q$ with coefficients in $R$. We identify $R=W_{1}(R)$. Let $\Phi_{R}$ be the canonical Frobenius endomorphism of either $W(R)$ or $W_{q}(R)$; we have $\Phi_{k}=\sigma_{k}=\sigma$. Let $R^{\left(p^{q}\right)}$ be $R$ but viewed as an $R$-algebra via the $q$-th power Frobenius endomorphism $\Phi_{R}^{q}: R \rightarrow R$. If $R$ is reduced, let $R^{\text {perf }}:=$ ind $\lim _{q \in \mathbf{N}} R^{\left(p^{q}\right)}$ be the perfection of $R$.

Let $\mathcal{M}\left(W_{q}(R)\right)$ be the abelian category whose objects are $W_{q}(R)$-modules endowed with $\Phi_{R}$-linear endomorphisms and whose morphisms are $W_{q}(R)$-linear maps that respect the $\Phi_{R^{-}}$ linear endomorphisms. We identify $\mathcal{M}\left(W_{q}(R)\right)$ with a full subcategory of $\mathcal{M}\left(W_{q+1}(R)\right)$ and thus we can define $\mathcal{M}(W(R)):=\bigcup_{q \in \mathbf{N}} \mathcal{M}\left(W_{q}(R)\right)$.

If $S$ is a $\operatorname{Spec}\left(\mathbf{F}_{p}\right)$-scheme, in a similar way we define $W_{q}(S), \Phi_{S}, \mathcal{M}\left(W_{q}(S)\right)$, and $\mathcal{M}(W(S))$. We view $W_{q}(S)$ as a scheme and by a $W_{q}(S)$-module we mean a quasi-coherent module over the structure ring sheaf $\mathcal{O}_{W_{q}(S)}$ of $W_{q}(S)$. The formal scheme $W(S)$ is used only as a notation. If $S=\operatorname{Spec}(R)$, then we identify canonically $\mathcal{M}\left(W_{q}(R)\right)=\mathcal{M}\left(W_{q}(S)\right)$ and $\mathcal{M}(W(R))=\mathcal{M}(W(S))$. If $t \in\{1, \ldots, q\}$ and $*(q)$ is a morphism of $\mathcal{M}\left(W_{q}(S)\right)$, let $*(t)$ be the morphism of $\mathcal{M}\left(W_{t}(S)\right)$ that is the tensorization of $*(q)$ with $W_{t}(S)$. Let $S^{\text {top }}$ be the topological space underlying $S$. All crystals over $S$ (i.e. all crystals on Berthelot's crystalline site $\operatorname{CRIS}\left(S / \operatorname{Spec}\left(\mathbf{Z}_{p}\right)\right)$ ) are in locally free sheaves of finite rank. An $F$-crystal $\mathfrak{C}$ over $S$ comprises from a crystal $\mathfrak{M}$ over $S$ and an isogeny $\Phi_{S}^{*}(\mathfrak{M}) \rightarrow \mathfrak{M}$ of crystals over $S$; let $h_{\mathfrak{C}} \in \mathbf{N} \cup\{0\}$ be the smallest number such that $p^{h_{\mathfrak{E}}}$ annihilates the cokernel of this isogeny. We 
identify an $F$-crystal (respectively an $F$-isocrystal) over $\operatorname{Spec}(k)$ with a latticed $F$-isocrystal $(M, \varphi)$ over $k$ that has the property that $\varphi(M) \subseteq M$ (respectively with an $F$-isocrystal over $k$ as defined in Section 1). The pulls back of $F$-crystals $\mathfrak{C}$ and $\mathfrak{C}_{*}$ over $S$ to an $S$-scheme $S_{1}$ (respectively to an affine $S$-scheme $\operatorname{Spec}\left(R_{1}\right)$ ) are denoted by $\mathfrak{C}_{S_{1}}$ and $\mathfrak{C}_{* S_{1}}$ (respectively by $\mathfrak{C}_{R_{1}}$ and $\left.\mathfrak{C}_{* R_{1}}\right)$.

Let $(M, \varphi, G)$ be a latticed $F$-isocrystal with a group over $k$. We refer to $M$ as its $W(k)$ module. Let $r_{M} \in \mathbf{N} \cup\{0\}$ be the rank of $M$. If $f_{1}$ and $f_{2}$ are two $\mathbf{Z}$-endomorphisms of either $M$ or $M\left[\frac{1}{p}\right]$, let $f_{1} f_{2}:=f_{1} \circ f_{2}$. Two Z Z-endomorphisms of $M$ are said to be congruent $\bmod p^{q}$ if their reductions $\bmod p^{q}$ coincide. Let $M^{*}:=\operatorname{Hom}(M, W(k))$. Let

$$
\mathcal{T}(M):=\bigoplus_{t, u \in \mathbf{N} \cup\{0\}} M^{\otimes t} \otimes_{W(k)} M^{* \otimes u} .
$$

We denote also by $\varphi$ the $\sigma$-linear automorphism of $\mathcal{T}(M)\left[\frac{1}{p}\right]$ that takes $f \in M^{*}\left[\frac{1}{p}\right]$ into $\sigma f \varphi^{-1} \in M^{*}\left[\frac{1}{p}\right]$ and that acts on $\mathcal{T}(M)\left[\frac{1}{p}\right]$ in the natural tensor product way. The canonical identification $\operatorname{End}\left(M\left[\frac{1}{p}\right]\right)=M\left[\frac{1}{p}\right] \otimes_{B(k)} M^{*}\left[\frac{1}{p}\right]$ is compatible with the $\varphi$ actions (see 1.1(a) for the action of $\varphi$ on $\left.\operatorname{End}\left(M\left[\frac{1}{p}\right]\right)\right)$. If $O$ is either a free $W(k)$-submodule or a $B(k)$-vector subspace of $\mathcal{T}(M)\left[\frac{1}{p}\right]$ such that $\varphi(M) \subseteq M$, then we denote also by $\varphi$ the $\sigma$-linear endomorphism of $O$ induced by $\varphi$. The $W(k)$-span of tensors $v_{1}, \ldots, v_{n} \in \mathcal{T}(M)\left[\frac{1}{p}\right]$ is denoted by $\left\langle v_{1}, \ldots, v_{n}\right\rangle$. The latticed $F$-isocrystal $\left(M^{*}, \varphi\right)$ over $k$ is called the dual of $(M, \varphi)$. We emphasize that the pair $\left(M^{*}, \varphi\right)$ involves no Tate twist. A bilinear form on $M$ is called perfect if it defines naturally a $W(k)$-linear isomorphism $M \stackrel{\sim}{\longrightarrow} M^{*}$.

Let $\widetilde{G}_{B(k)}$ be a connected subgroup of $\mathbf{G L}_{M\left[\frac{1}{p}\right]}$. As $\varphi$ is a $\sigma$-linear automorphism of $M\left[\frac{1}{p}\right]$, the group $\left\{\varphi \tilde{g} \varphi^{-1} \mid \tilde{g} \in \widetilde{G}_{B(k)}(B(k))\right\}$ is the group of $B(k)$-valued points of the unique connected subgroup of $\mathbf{G L}_{M\left[\frac{1}{p}\right]}$ that has $\varphi\left(\operatorname{Lie}\left(\widetilde{G}_{B(k)}\right)\right)$ as its Lie algebra (see [1, Ch. II, 7.1] for the uniqueness part). So as $\varphi$ normalizes $\operatorname{Lie}\left(G_{B(k)}\right)$, for $g \in G(B(k))$ we have $\varphi g \varphi^{-1} \in G(B(k))$; in what follows this fact is used without any extra comment.

In this paragraph we assume $\varphi(M) \subseteq M$. We also refer to $(M, \varphi, G)$ as an $F$-crystal with a group over $k$. The Hodge slopes of $(M, \varphi)$ (see [22, 1.2]) are the non-negative integers $h_{1}, \ldots, h_{r_{M}}$ such that the torsion $W(k)$-module $M / \varphi(M)$ is isomorphic to $\bigoplus_{i=1}^{r_{M}} W(k) /\left(p^{h_{i}}\right)$. If $O$ is a $W(k)$-submodule of $M$ such that $\varphi(O) \subseteq O$, we denote also by $\varphi$ the $\sigma$-linear endomorphism of $M / O$ induced by $\varphi$. We refer to the triple $\left(M / p^{q} M, \varphi, G_{W_{q}(k)}\right)$ as the reduction $\bmod p^{q}$ of $(M, \varphi, G)$. If $G=\mathbf{G L}_{M}$, then often we do not mention $G$ and $G_{W_{q}(k)}$ and we omit "with a group". The reduction $\left(M / p^{q} M, \varphi\right) \bmod p^{q}$ of $(M, \varphi)$ is an object of $\mathcal{M}\left(W_{q}(k)\right)$.

If $a, b \in \mathbf{Z}$ with $b \geqslant a$, let $S(a, b):=\{a, a+1, \ldots, b\}$. If $l \in \mathbf{N}$, if $*$ is a small letter, and if $\left(*_{1}, \ldots, *_{l}\right)$ is an $l$-tuple which is either an element of $\mathbf{Z}^{l}$ or an ordered $W(k)$-basis of some $W(k)$-module, then we define $*_{t}$ for any $t \in \mathbf{Z}$ via the rule: $*_{t}:=*_{u}$, where $u \in$ $\{1, \ldots, l\} \cap(t+l \mathbf{Z})$. If $x \in \mathbf{R}$, let $[x]$ be the greatest integer of the interval $(-\infty, x]$.

\subsubsection{Conventions on stratifications}

Let $K$ be a field. By a stratification $\mathcal{S}$ of a reduced $\operatorname{Spec}(K)$-scheme $X$ (in potentially an infinite number of strata), we mean that:

(i) for any field $L$ that is either $K$ or an algebraically closed field that contains $K$, a set $\mathcal{S}_{L}$ of disjoint reduced, locally closed subschemes of $X_{L}$ is given such that each point of $X_{L}$ with values in an algebraic closure of $L$ factors through some element of $\mathcal{S}_{L}$; 
(ii) if $i_{12}: L_{1} \hookrightarrow L_{2}$ is an embedding between two fields as in (a), then the reduced scheme of the pull back to $L_{2}$ of any member of $\mathcal{S}_{L_{1}}$, is an element of $\mathcal{S}_{L_{2}}$; so we have a natural pull back injective map $\mathcal{S}\left(i_{12}\right): \mathcal{S}_{L_{1}} \hookrightarrow \mathcal{S}_{L_{2}}$.

If the inductive limit of all maps $\mathcal{S}\left(i_{12}\right)$ exists (respectively does not exist) in the category of sets, then we say that the stratification $\mathcal{S}$ has a class which is (respectively is not) a set. Each element of some set $\mathcal{S}_{L}$ is referred as a stratum of $\mathcal{S}$. We say $\mathcal{S}$ satisfies the purity property if for any field $L$ as in (a), every element of $\mathcal{S}_{L}$ is an affine $X_{L}$-scheme. ${ }^{1}$ Thus $\mathcal{S}$ satisfies the purity property if and only if each stratum of it is an affine $X$-scheme. If all maps $\mathcal{S}\left(i_{12}\right)$ 's are bijections, then we identify $\mathcal{S}$ with $\mathcal{S}_{K}$ and we say $\mathcal{S}$ is of finite type.

\subsection{Definitions and simple properties}

In this section we introduce few notions and simple properties that pertain naturally to latticed $F$-isocrystals.

\subsubsection{Complements to 1.1}

(a) A morphism (respectively an isogeny) between two latticed $F$-isocrystals $\left(M_{1}, \varphi_{1}\right)$ and $\left(M_{2}, \varphi_{2}\right)$ over $k$ is a $W(k)$-linear map (respectively isomorphism) $f: M_{1}\left[\frac{1}{p}\right] \rightarrow M_{2}\left[\frac{1}{p}\right]$ such that $f \varphi_{1}=\varphi_{2} f$ and $f\left(M_{1}\right) \subseteq M_{2}$. If $f$ is an isogeny, then by its degree we mean $p^{l}$, where $l$ is the length of the artinian $W(k)$-module $M_{2} / f\left(M_{1}\right)$.

(b) By a latticed F-isocrystal with a group and an emphasized family of tensors over $k$ we mean a quadruple

$$
\left(M, \varphi, G,\left(t_{\alpha}\right)_{\alpha \in \mathcal{J}}\right),
$$

where $(M, \varphi, G)$ is a latticed $F$-isocrystal with a group over $k$, where $\mathcal{J}$ is a set of indices, and where $t_{\alpha} \in \mathcal{T}(M)$ is a tensor that is fixed by both $\varphi$ and $G$, such that $G_{B(k)}$ is the subgroup of $\mathbf{G L}_{M\left[\frac{1}{p}\right]}$ that fixes $t_{\alpha}$ for all $\alpha \in \mathcal{J}$. If $\left(M_{1}, \varphi_{1}, G_{1},\left(t_{1 \alpha}\right)_{\alpha \in \mathcal{J}}\right)$ and $\left(M_{2}, \varphi_{2}, G_{2},\left(t_{2 \alpha}\right)_{\alpha \in \mathcal{J}}\right)$ are two latticed $F$-isocrystals with a group and an emphasized family of tensors (indexed by the same set $\mathcal{J}$ ) over $k$, by an isomorphism between them we mean an isomorphism $f:\left(M_{1}, \varphi_{1}, G_{1}\right) \stackrel{\sim}{\longrightarrow}\left(M_{2}, \varphi_{2}, G_{2}\right)$ such that the $W(k)$-linear isomorphism $\mathcal{T}\left(M_{1}\right) \stackrel{\sim}{\longrightarrow} \mathcal{T}\left(M_{2}\right)$ induced by $f$, takes $t_{1 \alpha}$ into $t_{2 \alpha}$ for all $\alpha \in \mathcal{J}$.

(c) By a principal bilinear quasi-polarized latticed $F$-isocrystal with a group over $k$ we mean a quadruple $\left(M, \varphi, G, \lambda_{M}\right)$, where $(M, \varphi, G)$ is a latticed $F$-isocrystal with a group over $k$ and where $\lambda_{M}: M \otimes_{W(k)} M \rightarrow W(k)$ is a perfect bilinear form with the properties that the $W(k)$-span of $\lambda_{M}$ is normalized by $G$ and that there exists $c \in \mathbf{Z}$ such that we have $\lambda_{M}(\varphi(x), \varphi(y))=p^{c} \sigma\left(\lambda_{M}(x, y)\right)$ for all $x, y \in M$. We refer to $\lambda_{M}$ as a principal bilinear quasi-polarization of $(M, \varphi, G),(M, \varphi)$, and $\left(M\left[\frac{1}{p}\right], \varphi\right)$. Let $G^{0}$ be the Zariski closure in $\mathbf{G L}_{M}$ of the identity component of the subgroup of $G_{B(k)}$ that fixes $\lambda_{M}$. We refer to $\left(M, \varphi, G^{0}\right)$ as the latticed $F$-isocrystal with a group over $k$ of $\left(M, \varphi, G, \lambda_{M}\right)$. The quotient group $G_{B(k)} / G_{B(k)}^{0}$ is either trivial or isomorphic to $\mathbf{G}_{m}$.

By an isomorphism between two principal bilinear quasi-polarized latticed $F$-isocrystals with a group $\left(M_{1}, \varphi_{1}, G_{1}, \lambda_{M_{1}}\right)$ and $\left(M_{2}, \varphi_{2}, G_{2}, \lambda_{M_{2}}\right)$ over $k$ we mean an isomorphism $f:\left(M_{1}, \varphi_{1}, G_{1}\right) \stackrel{\sim}{\longrightarrow}\left(M_{2}, \varphi_{2}, G_{2}\right)$ such that we have $\lambda_{M_{1}}(x, y)=\lambda_{M_{2}}(f(x), f(y))$ for all $x$, $y \in M_{1}$. We speak also about principal bilinear quasi-polarized latticed $F$-isocrystals with a group and an emphasized family of tensors over $k$ and about isomorphisms between them; notation $\left(M, \varphi, G,\left(t_{\alpha}\right)_{\alpha \in \mathcal{J}}, \lambda_{M}\right)$.

\footnotetext{
${ }^{1}$ This is a more practical, refined, and general definition than any other one that relies on codimension 1 statements on complements. See Remark 6.3(a) below.
} 
If the form $\lambda_{M}$ is alternating, we drop the word bilinear (i.e. we speak about principal quasipolarized latticed $F$-isocrystals with a group over $k$, etc.).

(d) We say the $W$-condition holds for the latticed $F$-isocrystal with a group $(M, \varphi, G)$ over $k$ if there exists a direct sum decomposition $M=\bigoplus_{i=a}^{b} \widetilde{F}^{i}(M)$, where $a, b \in \mathbf{Z}$ with $b \geqslant a$, such that $M=\bigoplus_{i=a}^{b} \varphi\left(p^{-i} \widetilde{F}^{i}(M)\right)$ and the cocharacter $\mu: \mathbf{G}_{m} \rightarrow \mathbf{G L}_{M}$ defined by the property that $\beta \in \mathbf{G}_{m}(W(k))$ acts on $\widetilde{F}^{i}(M)$ through $\mu$ as the multiplication by $\beta^{-i}$, factors through $G$. In such a case we also refer to $(M, \varphi, G)$ as a $p$-divisible object with a group over $k$. We refer to the factorization $\mu: \mathbf{G}_{m} \rightarrow G$ of $\mu$ as a Hodge cocharacter of $(M, \varphi, G)$. For $i \in S(a, b)$ let $F^{i}(M):=\bigoplus_{j=b}^{i} \widetilde{F}^{j}(M)$. We refer to the decreasing and exhaustive filtration $\left(F^{i}(M)\right)_{i \in S(a, b)}$ of $M$ as a lift of $(M, \varphi, G)$. If $G=\mathbf{G L}_{M}$, we also refer to $(M, \varphi)$ as a $p$-divisible object over $k$.

Here " $W$ " stands to honor [42, p. 512] while the notion " $p$-divisible object" is a natural extrapolation of the terminology "object" introduced in [11, §2].

(e) By the shifting number (to be abbreviated as the s-number) of a latticed $F$-isocrystal $(M, \varphi)$ over $k$ we mean the smallest number $s \in \mathbf{N} \cup\{0\}$ such that $\varphi\left(p^{s} M\right) \subseteq M$ (equivalently such that $\varphi(M) \subseteq p^{-s} M$ ). By the greatest Hodge slope (to be abbreviated as the $h$-number $)$ of $(M, \varphi)$ we mean the greatest Hodge slope $h$ of $\left(M, p^{s} \varphi\right)$, i.e. the unique number $h \in \mathbf{N} \cup\{0\}$ such that we have $p^{h-s} M \subseteq \varphi(M)$ and $p^{h-s-1} M \nsubseteq \varphi(M)$.

We have $s=0$ if and only if $(M, \varphi)$ is an $F$-crystal over $k$; in this case $h$ is the number $h_{(M, \varphi)}$ defined in 2.1. We have $s=0$ and $h \in\{0,1\}$ if and only if $(M, \varphi)$ is a Dieudonné module over $k$.

Let $s^{*}$ and $h^{*}$ be the $s$-number and the $h$-number (respectively) of $\left(M^{*}, \varphi\right)$. We have $\varphi\left(M^{*}\right)=\varphi(M)^{*} \subseteq p^{s-h} M^{*}$ but $\varphi\left(M^{*}\right) \nsubseteq p^{s-h+1} M^{*}$. Thus $s^{*}=\max \{0, h-s\}$. As $(M, \varphi)$ is the dual of $\left(M^{*}, \varphi\right)$, we also have $s=\max \left\{0, h^{*}-s^{*}\right\}$. So if $s=0$, then $s^{*}=h$ and $h^{*} \in S(0, h)$. If $s>0$, then $s=h^{*}-s^{*}$ and thus $h^{*}=s+s^{*}=\max (s, h)$.

If $s=0$, then the $s$-number and the $h$-number of $(\operatorname{End}(M), \varphi)=(M, \varphi) \otimes\left(M^{*}, \varphi\right)$ are at most $s+s^{*}=h$ and $h+h^{*} \leqslant 2 h$ (respectively).

\subsubsection{DeFinitions. -}

(a) Let $(M, \varphi)$ be a $p$-divisible object $(M, \varphi)$ over $k$. We say $(M, \varphi)$ is a cyclic DieudonnéFontaine p-divisible object over $k$ if there exists a $W(k)$-basis $\left\{e_{1}, \ldots, e_{r_{M}}\right\}$ of $M$ such that for $i \in S\left(1, r_{M}\right)$ we have an identity $\varphi\left(e_{i}\right)=p^{n_{i}} e_{i+1}$, where $n_{1}, \ldots, n_{r_{M}}$ are integers that are either all non-negative or all non-positive. We refer to $\left\{e_{1}, \ldots, e_{r_{M}}\right\}$ as a standard $W(k)$-basis of $(M, \varphi)$.

We say $(M, \varphi)$ is an elementary Dieudonné-Fontaine p-divisible object over $k$ if it is a cyclic Dieudonné-Fontaine $p$-divisible object over $k$ that is not the direct sum of two or more non-trivial cyclic Dieudonné-Fontaine $p$-divisible objects over $k$.

We say $(M, \varphi)$ is an elementary Dieudonné p-divisible object over $k$ if there exists a $W(k)$-basis $\left\{e_{1}, \ldots, e_{r_{M}}\right\}$ of $M$ such that for $i \in S\left(2, r_{M}\right)$ we have an identity $\varphi\left(e_{i}\right)=$ $e_{i+1}$ and moreover $\varphi\left(e_{1}\right)=p^{n_{1}} e_{2}$ for some integer $n_{1}$ that is relatively prime to $r_{M}$.

We say $(M, \varphi)$ is a Dieudonné-Fontaine (respectively a Dieudonné) p-divisible object over $k$ if it is a direct sum of elementary Dieudonné-Fontaine (respectively of elementary Dieudonné) $p$-divisible objects over $k$.

(b) By the Dieudonné-Fontaine torsion (respectively volume) of a latticed $F$-isocrystal $(M, \varphi)$ over $k$ we mean the smallest number

$$
\mathbf{T}(M, \varphi) \in \mathbf{N} \cup\{0\}
$$

(respectively $\mathbf{V}(M, \varphi) \in \mathbf{N} \cup\{0\}$ ) such that there exists a Dieudonné-Fontaine $p$-divisible object $\left(M_{1}, \varphi_{1}\right)$ over $\bar{k}$ for which we have an isogeny 
$f:\left(M_{1}, \varphi_{1}\right) \hookrightarrow\left(M \otimes_{W(k)} W(\bar{k}), \varphi \otimes \sigma_{\bar{k}}\right)$ with the property that $p^{\mathbf{T}(M, \varphi)} M \subseteq f\left(M_{1}\right)$ (respectively that $M / f\left(M_{1}\right)$ has length $\mathbf{V}(M, \varphi)$ ). By replacing Dieudonné-Fontaine with Dieudonné, in a similar way we define the Dieudonné torsion $\mathbf{T}_{+}(M, \varphi) \in \mathbf{N} \cup\{0\}$ and the Dieudonné volume $\mathbf{V}_{+}(M, \varphi) \in \mathbf{N} \cup\{0\}$ of $(M, \varphi)$.

2.2.2.1. Remarks. - (a) Any (elementary) Dieudonné $p$-divisible object over $k$ is also an (elementary) Dieudonné-Fontaine $p$-divisible object over $k$. Moreover, any Dieudonné-Fontaine $p$-divisible object over $k$ is definable over $\mathbf{F}_{p}$.

(b) The existence of $\mathbf{V}_{+}(M, \varphi)$ (and thus also of $\mathbf{V}(M, \varphi), \mathbf{T}_{+}(M, \varphi)$, and $\mathbf{T}(M, \varphi)$ ) is equivalent to Dieudonné's classification of $F$-isocrystals over $\bar{k}$. This and the fact that suitable reductions (modulo powers of $p$ ) of $p$-divisible objects over $k$ are studied systematically for the first time in [14,15], explains our terminology.

(c) Classically one works only with Dieudonné $p$-divisible objects (as they are uniquely determined by their Newton polygons) and with Dieudonné volumes (as they keep track of degrees of isogenies); see $[7,28,6,10]$, etc. But working with Dieudonné-Fontaine $p$-divisible objects and torsions one can get considerable improvements for many practical calculations or upper bounds (like the ones we will encounter in Section 3).

2.2.3. LemmA. - Let $K$ be an algebraically closed field that contains $k$. Let $(M, \varphi)$ be a Dieudonné-Fontaine p-divisible object over $k$ with the property that $\varphi(M) \subseteq M$. Let $h$ be the $h$-number of $(M, \varphi)$, let $e_{M}:=\max \left\{r_{M},\left[\frac{r_{M}^{2}}{4}\right]\right\}$, let $k_{1}$ be the composite field of $k$ and $\mathbf{F}_{p^{r} M}$, and let $m \in \mathbf{N}$. We have the following two properties:

(a) For any endomorphism $f_{h e_{M}+m}$ of $\left(M \otimes_{W(k)} W_{h e_{M}+m}(K), \varphi \otimes \sigma_{K}\right)$, the reduction $f_{m} \bmod p^{m}$ of $f_{h e_{M}+m}$ is the scalar extension of an endomorphism of $\left(M \otimes_{W(k)}\right.$ $\left.W_{m}\left(k_{1}\right), \varphi \otimes \sigma_{k_{1}}\right)$. If $(M, \varphi)$ is a Dieudonné p-divisible object over $k$, then the previous sentence holds with $e_{M}$ being substituted by $r_{M}$.

(b) Each endomorphism of $\left(M \otimes_{W(k)} W(K), \varphi \otimes \sigma_{K}\right)$ is the scalar extension of an endomorphism of $\left(M \otimes_{W(k)} W\left(k_{1}\right), \varphi \otimes \sigma_{k_{1}}\right)$.

Proof. - We write $(M, \varphi)=\bigoplus_{i=1}^{s}\left(M_{i}, \varphi\right)$ as a direct sum of elementary Dieudonné-Fontaine $p$-divisible objects over $k$. Let $\left\{e_{1}^{(i)}, \ldots, e_{r_{M_{i}}}^{(i)}\right\}$ be a standard $W(k)$-basis of $\left(M_{i}, \varphi\right)$. We check that (a) holds. Let $i_{0} \in S(1, s)$ and let $j_{0} \in S\left(1, r_{M_{i_{0}}}\right)$. We write $f_{h e_{M}+m}\left(e_{j_{0}}^{\left(i_{0}\right)} \otimes 1\right)=$ $\sum_{i=1}^{s} \sum_{j=1}^{r_{M_{i}}} e_{j}^{(i)} \otimes \beta_{j_{0} j}^{\left(i_{0} i\right)}$, where all $\beta_{j_{0} j}^{\left(i_{0} i\right)}$,s belong to $W_{h e_{M}+m}(K)$. Let $r_{M_{i_{0} i}}:=$ l.c.m. $\left\{r_{M_{i_{0}}}, r_{M_{i}}\right\}$; it is a divisor of $r_{M}$ !. If $i=i_{0}$, then $r_{M_{i_{0}}}=r_{M_{i_{0}}} \leqslant r_{M} \leqslant e_{M}$. If $i \neq i_{0}$, then $r_{M_{i_{0}}}+r_{M_{i}} \leqslant r_{M}$ and thus we have $r_{M_{i_{0} i}} \leqslant r_{M_{i_{0}}} r_{M_{i}} \leqslant\left[\frac{r_{M}^{2}}{4}\right] \leqslant e_{M}$.

As $f_{h e_{M}+m}\left(\varphi^{r_{M_{i} i}}\left(e_{j_{0}}^{\left(i_{0}\right)}\right) \otimes 1\right)=\left(\varphi \otimes \sigma_{K}\right)^{r_{M_{i} i}}\left(f_{h e_{M}+m}\left(e_{j_{0}}^{\left(i_{0}\right)} \otimes 1\right)\right)$, we have an equality

$$
p^{m_{j_{0}}^{\left(i_{0} i\right)}} \beta_{j_{0} j}^{\left(i_{0} i\right)}=p^{q_{j}^{\left(i_{0} i\right)}} \sigma_{K}^{r_{M_{i} i} i}\left(\beta_{j_{0} j}^{\left(i_{0} i\right)}\right) \in W_{h e_{M}+m}(K)
$$

where $m_{j_{0}}^{\left(i_{0} i\right)} \in \mathbf{N} \cup\{0\}$ is such that $\varphi^{r_{M_{i_{0} i}}}\left(e_{j_{0}}^{\left(i_{0}\right)}\right)=p^{m_{j_{0}}^{\left(i_{0} i\right)}} e_{j_{0}}^{\left(i_{0}\right)}$ and where $q_{j}^{\left(i_{0} i\right)} \in \mathbf{N} \cup\{0\}$ is such that $\varphi^{r_{M_{i_{0} i}}}\left(e_{j}^{(i)}\right)=p^{q_{j}^{\left(i_{0} i\right)}} e_{j}^{(i)}$. The numbers $m_{j_{0}}^{\left(i_{0} i\right)}$ and $q_{j}^{\left(i_{0} i\right)}$ are at most $h r_{M_{i_{0} i}}$ and so at most $h e_{M}$. Let $s_{j_{0} j}^{\left(i_{0} i\right)} \in S\left(0, h e_{M}+m\right)$ be the unique number such that we can write $\beta_{j_{0} j}^{\left(i_{0} i\right)}=p^{s_{j_{0} j}^{\left(i_{0} i\right)}} \tilde{\beta}_{j_{0} j}^{\left(i_{0} i\right)}$, with $\tilde{\beta}_{j_{0} j}^{\left(i_{0} i\right)} \in \mathbf{G}_{m}\left(W_{h e_{M}+m}(K)\right)$. From (1) we easily get that

$$
t_{j_{0} j}^{\left(i_{0} i\right)}:=\min \left\{h e_{M}+m, m_{j_{0}}^{\left(i_{0} i\right)}+s_{j_{0} j}^{\left(i_{0} i\right)}\right\} \quad \text { equals to } \min \left\{h e_{M}+m, q_{j}^{\left(i_{0} i\right)}+s_{j_{0} j}^{\left(i_{0} i\right)}\right\}
$$


and that $\sigma_{K}^{r_{M_{i} i}}\left(\tilde{\beta}_{j_{0} j}^{\left(i_{0} i\right)}\right)$ and $\tilde{\beta}_{j_{0} j}^{\left(i_{0} i\right)}$ coincide $\bmod p^{h e_{M}+m-t_{j_{0} j}^{\left(i_{0} i\right)}}$. Thus $\tilde{\beta}_{j_{0} j}^{\left(i_{0} i\right)} \bmod p^{h e_{M}+m-t_{j_{0} j}^{\left(i_{0} i\right)}}$ belongs to $W_{h e_{M}+m-t_{j_{0} j}^{\left(i_{0} i\right)}}\left(\mathbf{F}_{p^{r_{M_{i} i}}}\right)$ and therefore $\beta_{j_{0} j}^{\left(i_{0} i\right)} \bmod p^{h e_{M}+m-t_{j_{0} j}^{\left(i_{0} i\right)}+s_{j_{0} j}^{\left(i_{0} i\right)}}$ belongs to $W_{h e_{M}+m-t_{j_{0} j}^{\left(i_{0} i\right)}+s_{j_{0} j}^{\left(i_{0} i\right)}\left(\mathbf{F}_{p}{ }^{{ }{ } i_{i_{0}} i}\right)}$. As $\quad-m_{j_{0}}^{\left(i_{0} i\right)} \leqslant-t_{j_{0} j}^{\left(i_{0} i\right)}+s_{j_{0} j}^{\left(i_{0} i\right)}$, we get that $\beta_{j_{0} j}^{\left(i_{0} i\right)} \bmod p^{h e_{M}+m-m_{j_{0}}^{\left(i_{0} i\right)}}$ belongs to $W_{h e_{M}+m-m_{j_{0}}^{\left(i_{0} i\right)}}\left(\mathbf{F}_{p^{{ }}{ } i_{i_{0} i}}\right)$ and thus also to $W_{h e_{M}+m-m_{j_{0}}^{\left(i_{0} i\right)}}\left(k_{1}\right)$. So due to the inequality $m \leqslant h e_{M}+m-m_{j_{0}}^{\left(i_{0} i\right)}$, we have $f_{m}\left(e_{j_{0}}^{\left(i_{0}\right)} \otimes 1\right) \in$ $M \otimes_{W(k)} W_{m}\left(k_{1}\right)$ for any pair $\left(i_{0}, j_{0}\right) \in S(1, s) \times S\left(1, r_{M_{i_{0}}}\right)$. Thus $f_{m}$ is the scalar extension of an endomorphism of $\left(M \otimes_{W(k)} W_{m}\left(k_{1}\right), \varphi \otimes \sigma_{k_{1}}\right)$.

If $(M, \varphi)$ is a Dieudonne $p$-divisible object over $k$, then the Hodge slopes of $\left(M_{i_{0}}, \varphi\right)$ are $0, \ldots, 0$, and some integer in $S(0, h)$; thus $m_{j_{0}}^{\left(i_{0} i\right)} \leqslant h \frac{r_{M_{i_{0} i}}}{r_{M_{i}}} \leqslant h r_{M_{i}} \leqslant h r_{M}$. A similar argument shows that $q_{j}^{\left(i_{0} i\right)} \leqslant h r_{M}$. Thus in the previous paragraph we can substitute $e_{M}$ by $r_{M}$. So (a) holds.

Part (b) follows from (a) by taking $m \rightarrow \infty$.

\subsubsection{Deviations of tuples}

Let $l \in \mathbf{N}$. Let $\tau=\left(n_{1}, \ldots, n_{l}\right) \in \mathbf{Z}^{l}$.

(a) Suppose $\sum_{i=1}^{l} n_{i}$ is non-negative (respectively is non-positive). Let $P(\tau)$ be the set of pairs $(t, u)$, where $t \in S(1, l)$ and $u \in S(t, l+t-1)$ have the property that all sums $\sum_{i=v}^{u} n_{i}$ with $v \in S(t, u)$ are non-positive (respectively are non-negative). By the non-negative (respectively the non-positive) sign deviation of $\tau$ we mean the non-negative integer $\max \left\{0,-\sum_{i=t}^{u} n_{i} \mid(t, u) \in P(\tau)\right\}$ (respectively $\max \left\{0, \sum_{i=t}^{u} n_{i} \mid\right.$ $(t, u) \in P(\tau)\})$.

(b) If $\sum_{i=1}^{l} n_{i}$ is non-negative (respectively is non-positive), then by the non-negative (respectively the non-positive) value deviation of $\tau$ we mean the absolute value of the sum of all non-positive (respectively of all non-negative) entries of $\tau$. As a convention, this sum is 0 if $\tau$ has no non-positive (respectively no non-negative) entries.

(c) If $\sum_{i=1}^{l} n_{i}$ is positive (respectively is negative), then by the sign deviation $\mathbf{S} \tau$ of $\tau$ we mean its non-negative (respectively its non-positive) sign deviation. If $\sum_{i=1}^{l} n_{i}=0$, then by the sign deviation $\mathbf{S} \tau$ of $\tau$ we mean the smaller of its non-negative and non-positive sign deviations. We also use this definition with (sign, $\mathbf{S}$ ) replaced by (value, $\mathbf{W}$ ).

Samples: $\mathbf{S}(-1,1,-1,-1,1,1,0,-1)=1+1=2, \mathbf{W}(-1,1,-1,-1,1,1,0,-1)=3$, $\mathbf{S}(1,1,-2,1,3)=\mathbf{W}(1,1,-2,1,3)=2$, and $\mathbf{S}(-1,1,-1)=\mathbf{W}(-1,1,-1)=1$.

\subsection{Inequalities}

Let $(M, \varphi)$ be a latticed $F$-isocrystal over $k$. Obviously $\mathbf{V}(M, \varphi) \leqslant \mathbf{V}_{+}(M, \varphi)$ and $\mathbf{T}(M, \varphi) \leqslant \mathbf{T}_{+}(M, \varphi)$. Moreover we have

$$
\mathbf{T}(M, \varphi) \leqslant \mathbf{V}(M, \varphi) \leqslant \mathbf{T}(M, \varphi) r_{M}
$$

and the same inequalities hold with $(\mathbf{T}, \mathbf{V})$ being replaced with $\left(\mathbf{T}_{+}, \mathbf{V}_{+}\right)$.

2.3.1. LemmA. - Let $\tau=\left(n_{1}, \ldots, n_{r_{M}}\right) \in \mathbf{Z}^{r_{M}}$. Suppose there exists a $W(k)$-basis $\left\{e_{1}, \ldots, e_{r_{M}}\right\}$ of $M$ such that for $i \in S\left(1, r_{M}\right)$ we have $\varphi\left(e_{i}\right)=p^{n_{i}} e_{i+1}$. Then we have the following sequence of three inequalities

$$
\mathbf{T}(M, \varphi) \leqslant \mathbf{S} \tau \leqslant \mathbf{W} \tau \leqslant\left|n_{1}\right|+\left|n_{2}\right|+\cdots+\left|n_{r_{M}}\right| .
$$

$4^{\mathrm{e}}$ SÉRIE - TOME $39-2006-\mathrm{N}^{\circ} 2$ 
Proof. - The second and the third inequalities follow from their very definitions.

We check the first inequality of (2) only in the case when $\sum_{i=1}^{r_{M}} n_{i}>0$ and at least one entry $n_{i}$ is negative, as in all other cases the first inequality of (2) is checked in the same way. We perform the following type of operation.

Let $u \in \mathbf{N} \cup\{0\}$ be the greatest number such that there exists $t \in S\left(1, r_{M}\right)$ with the property that $n_{t-v, t}:=\sum_{i=t-v}^{t} n_{i}$ is non-positive for all $v \in S(0, u)$; we have $n_{t+1}>0$, $n_{t-u-1}>0$, and $\sum_{i=t-u-1}^{t} n_{i}>0$. For $v \in S(0, u)$ we replace $e_{t-v}$ by $\tilde{e}_{t-v}:=p^{-n_{t-v, t}} e_{t-v}$. Up to a cyclic rearrangement of $\tau$, we can assume $t-u=1$; so $t=1+u$ and $n_{r_{M}}=$ $n_{0}=n_{t-u-1}>0$. The $r_{M}$-tuple $\left(\tilde{e}_{1}, \ldots, \tilde{e}_{u+1}, e_{u+2}, \ldots, e_{r_{M}}\right)$ is mapped by $\varphi$ into the $r_{M}$-tuple $\left(\tilde{e}_{2}, \ldots, \tilde{e}_{u+1}, e_{u+2}, p^{n_{u+2}} e_{u+3}, \ldots, p^{n_{r_{M}-1}} e_{r_{M}}, p^{\sum_{i=0}^{u+1} n_{i}} \tilde{e}_{1}\right)$. We have $\sum_{i=0}^{u+1} n_{i}=$ $\sum_{i=t-u-1}^{t} n_{i}>0$. So if for all $i \in S\left(u+2, r_{M}-1\right)$ we have $n_{i} \geqslant 0$, then the pair $\left(\left\langle\tilde{e}_{1}, \ldots, \tilde{e}_{u+1}, e_{u+2}, \ldots, e_{r_{M}}\right\rangle, \varphi\right)$ is a cyclic Dieudonné-Fontaine $p$-divisible object over $k$ and we are done as by their very definitions, we have $-n_{t-v, t} \in S(0, \mathbf{S} \tau)$ and thus $p^{\mathbf{S} \tau}$ annihilates the quotient $W(k)$-module $M /\left\langle\tilde{e}_{1}, \ldots, \tilde{e}_{u+1}, e_{u+2}, \ldots, e_{r_{M}}\right\rangle$; if this is not the case, we next deal with the inoperated entries $n_{u+2}, \ldots, n_{r_{M}}$.

We repeat the operation as follows. Let $u_{1} \in \mathbf{N} \cup\{0\}$ be the greatest number such that there exists $t_{1} \in S\left(u+2, r_{M}\right)$ with the property that $n_{t_{1}-v_{1}, t_{1}}:=\sum_{i=t_{1}-v_{1}}^{t_{1}} n_{i}$ is non-positive for all $v_{1} \in S\left(0, u_{1}\right)$; we have $n_{t_{1}+1}>0, n_{t_{1}-u_{1}-1}>0$, and $\sum_{i=t_{1}-u_{1}-1}^{t_{1}} n_{i}>0$. Due to the "greatest" property of $u$ we have $t_{1}-u_{1}>u+2$. For $v_{1} \in S\left(0, u_{1}\right)$ we replace $e_{t_{1}-v_{1}}$ by $\tilde{e}_{t_{1}-v_{1}}:=p^{-n_{t_{1}-v_{1}, t_{1}}} e_{t_{1}-v_{1}}$ and we repeat the operation for the inoperated entries $n_{u+2}, n_{u+3}, \ldots, n_{t_{1}-u_{1}-1}, n_{t_{1}+1}, n_{t_{1}+2}, \ldots, n_{r_{M}}$. By induction on the number of remaining inoperated entries (they do not have to be indexed by a set of consecutive numbers in $S\left(1, r_{M}\right)$ ), we get that the first inequality of (2) holds.

2.3.2. Example. - If for $i \in S\left(1, r_{M}\right)$ we have $n_{i} \in\{-1,0,1\}$, then from (2) we get

$$
\mathbf{T}(M, \varphi) \leqslant \mathbf{W} \tau=\min \left\{n^{-}, n^{+}\right\}
$$

where $n^{-}$(respectively $n^{+}$) is the number of $i$ 's such that $n_{i}=-1$ (respectively such that $\left.n_{i}=1\right)$.

We now consider the case when $r_{M} \geqslant 3$ and $\left(n_{1}, n_{2}, \ldots, n_{r_{M}}\right)=(1,1, \ldots, 1,-1)$. So $(M, \varphi)$ has a unique slope $\frac{r_{M}-2}{r_{M}}$ that is positive. As $\varphi(M) \nsubseteq M$, we have $\mathbf{T}(M, \varphi) \geqslant 1$. But $\mathbf{T}(M, \varphi) \leqslant 1$, cf. (3). Thus $\mathbf{T}(M, \varphi)=1$. In fact $\left(\left\langle e_{1}, \ldots, p e_{r_{M}}\right\rangle, \varphi\right)$ is an elementary Dieudonné-Fontaine $p$-divisible object over $k$ whose Hodge slopes are $0,0,1, \ldots, 1$. It is easy to see that $\mathbf{T}_{+}(M, \varphi)=r_{M}-2$; so $\mathbf{T}_{+}(M, \varphi)>\mathbf{T}(M, \varphi)$ for $r_{M}>3$.

\subsection{Estimates}

Let $(M, \varphi)$ be a latticed $F$-isocrystal over $k$. Let $s$ and $h$ be the $s$-number and the $h$-number (respectively) of $(M, \varphi)$. Let $\mathcal{H}$ be the set of slopes of $\left(M\left[\frac{1}{p}\right], \varphi\right)$. If $\alpha \in \mathcal{H}$, we write $\alpha=\frac{a_{\alpha}}{b_{\alpha}}$, where $\left(a_{\alpha}, b_{\alpha}\right) \in \mathbf{Z} \times \mathbf{N}$ with g.c.d. $\left(a_{\alpha}, b_{\alpha}\right)=1$.

2.4.1. LemmA. - Suppose $k=\bar{k}$. Let $(a, b, c) \in \mathbf{N} \times(\mathbf{N} \cup\{0\}) \times(\mathbf{N} \cup\{0\})$. There exists a smallest number

$$
d(a, b, c) \in \mathbf{N} \cup\{0\}
$$

(respectively $\left.d_{+}(a, b, c) \in \mathbf{N} \cup\{0\}\right)$ such that for any latticed $F$-isocrystal $\mathfrak{C}$ over $k$ of rank $a$, $s$-number $b$, and h-number $c$, we have an inequality $\mathbf{T}(\mathfrak{C}) \leqslant d(a, b, c)$ (respectively $\mathbf{T}_{+}(\mathfrak{C}) \leqslant$ $\left.d_{+}(a, b, c)\right)$. In particular, for any element $g \in \mathbf{G L}_{M}(W(k))$ we have $\mathbf{T}(M, g \varphi) \leqslant d\left(r_{M}, s, h\right)$. 
Moreover upper bounds of $d(a, b, c)$ (respectively of $d_{+}(a, b, c)$ ) are effectively computable in terms of $a, b$, and $c$.

Proof. - As $\mathbf{T}(\mathfrak{C}) \leqslant \mathbf{T}_{+}(\mathfrak{C})$, it suffices to prove the lemma for $d_{+}(a, b, c)$. To ease the notations, we will assume that $\left(r_{M}, s, h\right)=(a, b, c)$ and that $(M, \varphi)=\mathfrak{C}$. We have

$$
\mathbf{T}_{+}(M, \varphi) \leqslant s\left(\max \left\{b_{\alpha} \mid \alpha \in \mathcal{H}\right\}-1\right)+\mathbf{T}_{+}\left(M, p^{s} \varphi\right) .
$$

To check this inequality we first remark that if $O$ is a $W(k)$-submodule of $M$ such that the pair $\left(O, p^{s} \varphi\right)$ is an elementary Dieudonné $p$-divisible object over $k$ and if $\left\{e_{1}, \ldots, e_{r_{O}}\right\}$ is a standard $W(k)$-basis of $\left(O, p^{s} \varphi\right)$ such that we have $\left(p^{s} \varphi\right)\left(e_{i}\right)=e_{i+1}$ for all $i \in S\left(1, r_{O}-1\right)$, then the following pair $\left(O^{\prime}, \varphi\right):=\left(\left\langle p^{r_{O} s-s} e_{1}, p^{r_{O}{ }^{s-2 s}} e_{2}, \ldots, p^{s} e_{r_{O}-1}, e_{r_{O}}\right\rangle, \varphi\right)$ is an elementary Dieudonné $p$-divisible object over $k$. As $\left(O\left[\frac{1}{p}\right], \varphi\right)$ is a simple $F$-isocrystal over $k$ whose unique slope belongs to $\mathcal{H}$, we have $r_{O} \leqslant \max \left\{b_{\alpha} \mid \alpha \in \mathcal{H}\right\}$. From this and the fact that $O / O^{\prime}$ is annihilated by $p^{s\left(r_{O}-1\right)}$, we easily get that (4) holds.

Thus it suffices to prove the existence of a number $d_{+}(a, b, c)$ that has all the required properties under the extra assumption $b=s=0$; as $s=0$, we have $\mathcal{H} \subseteq[0, h]$. We will use an induction on $a=r_{M}$. The case $a=1$ is trivial. To accomplish for $a \geqslant 2$ the inductive passage from $a-1=r_{M}-1$ to $a=r_{M}$, we consider two disjoint cases.

Case 1. Suppose the $F$-isocrystal $\left(M\left[\frac{1}{p}\right], \varphi\right)$ over $k$ is not simple. Let $\alpha \in \mathcal{H}$. We consider a short exact sequence $0 \rightarrow\left(M_{1}, \varphi\right) \rightarrow(M, \varphi) \rightarrow\left(M_{2}, \varphi\right) \rightarrow 0$ such that the $F$-isocrystal $\left(M_{2}\left[\frac{1}{p}\right], \varphi\right)$ over $k$ is simple of $\alpha$. For $i \in S(1,2)$, the $h$-number of $\left(M_{i}, \varphi\right)$ is at most $h$ and we have $r_{M_{i}}<a=r_{M}$. By induction, there exists $d_{i} \in \mathbf{N} \cup\{0\}$ that has upper bounds effectively computable in terms of $r_{M_{i}}$ and $c=h$ and such that there exists a $W(k)$-submodule $O_{i}$ of $M_{i}$ with the properties that $r_{O_{i}}=r_{M_{i}}$, that $p^{d_{i}} M_{i} \subseteq O_{i}$, and that $\left(O_{i}, \varphi\right)$ is a Dieudonné $p$-divisible object over $k$. The map $\sigma^{b_{\alpha}}-1_{W(k)}: W(k) \rightarrow W(k)$ is onto. This implies that $p^{a_{\alpha}} M_{1} \subseteq\left(\varphi^{b_{\alpha}}-p^{a_{\alpha}} 1_{M_{1}}\right)\left(M_{1}\right)$. Let $x \in O_{2}$ be such that $\varphi^{b_{\alpha}}(x)=p^{a_{\alpha}} x$ and $\varphi^{b_{\alpha}-1}(x) \in$ $\mathrm{O}_{2} \backslash p \mathrm{O}_{2}$. If $\tilde{x} \in M$ maps into $x$, then there exists $y \in M_{1}$ such that $\varphi^{b_{\alpha}}(y)-p^{a_{\alpha}}(y)$ is $p^{a_{\alpha}}\left[\varphi^{b_{\alpha}}(\tilde{x})-p^{a_{\alpha}}(\tilde{x})\right] \in p^{a_{\alpha}} M_{1}$. Thus $z:=-y+p^{a_{\alpha}} \tilde{x} \in M$ maps into $p^{a_{\alpha}} x$ and we have $\varphi^{b_{\alpha}}(z)=p^{a_{\alpha}}(z)$. By choosing $x$ to belong to a standard $W(k)$-basis of $\left(O_{2}, \varphi\right)$, we get that the monomorphism $i_{2}:\left(p^{a_{\alpha}} O_{2}, \varphi\right) \hookrightarrow\left(M_{2}, \varphi\right)$ lifts to a monomorphism $j_{2}:\left(p^{a_{\alpha}} O_{2}, \varphi\right) \hookrightarrow(M, \varphi)$. As $\left(O_{1}+j_{2}\left(p^{a_{\alpha}} O_{2}\right), \varphi\right)$ is a Dieudonné $p$-divisible object over $k$ and as $p^{d_{1}+d_{2}+a_{\alpha}}$ annihilates $M / O_{1}+j_{2}\left(p^{a_{\alpha}} O_{2}\right)$, we get

$$
\mathbf{T}_{+}(M, \varphi) \leqslant d_{1}+d_{2}+a_{\alpha} \leqslant d_{1}+d_{2}+h b_{\alpha} \leqslant d_{1}+d_{2}+h r_{M} .
$$

Case 2. Suppose the $F$-isocrystal $\left(M\left[\frac{1}{p}\right], \varphi\right)$ over $k$ is simple of slope $\alpha$. Thus $a=r_{M}=b_{\alpha}$. Let $\left\{e_{1}, \ldots, e_{a}\right\} \subseteq M$ be a $B(k)$-basis of $M\left[\frac{1}{p}\right]$ such that $e_{1} \in M \backslash p M$, for $i \in S(1, a-1)$ we have $\varphi\left(e_{i}\right)=e_{i+1}$, and $\varphi\left(e_{a}\right)=p^{a_{\alpha}} e_{1}$. For $t \in S(1, a)$, let $M_{t}:=\left\langle e_{1}, \ldots, e_{t}\right\rangle$ and $\widetilde{M}_{t}:=$ $M_{t}\left[\frac{1}{p}\right] \cap M$. We have $M_{1}=\widetilde{M}_{1}$ and $\widetilde{M}_{a}=M$.

2.4.1.1. ClaIM. - There exists a strictly increasing sequence $\left(c_{t}\right)_{t \in S(1, a)}$ of non-negative integers that depends only on $a=r_{M}$ and $c=h$, that is effectively computable, and that has the property that for any $t \in S(1, a)$ we have inclusions

$$
p^{c_{t}}\left(\widetilde{M}_{t}\right) \subseteq M_{t} \subseteq \widetilde{M}_{t}
$$

To check this claim we use induction on $t \in S(1, a)$. Taking $c_{1}:=0$, (6) holds for $t=1$. Suppose there exists a number $r \in S(1, a-1)$ such that (6) holds for $t \in S(1, r)$. We now check

$4^{\text {e }}$ SÉRIE - TOME $39-2006-\mathrm{N}^{\circ} 2$ 
that (6) holds for $t=r+1$. Thus we have to show that there exists an effectively computable natural number $c_{r+1}$ which is at least $c_{r}+1$ and for which we have

$$
e_{r+1}=\varphi\left(e_{r}\right) \notin p^{1+c_{r+1}} M+\widetilde{M}_{r}
$$

We write $e_{r+1}=\varphi\left(e_{r}\right)=p^{n_{r}} x_{r}+y_{r}$, where $x_{r} \in M \backslash p M, n_{r} \in \mathbf{N} \cup\{0\}$, and $y_{r} \in \widetilde{M}_{r}$. By our initial induction (on ranks), we can speak about an effectively computable number $d_{r} \in \mathbf{N}$ that is at least $\max \left\{d_{+}(r, 0, l) \mid l \in S(0, h)\right\}$. Let

$$
c_{r+1}:=c_{r}+d_{r}+r ! a h .
$$

We show that the assumption $n_{r}>c_{r+1}$ leads to a contradiction. Let $\widetilde{M}_{0}:=0$. Let $\tilde{e}_{r} \in \widetilde{M}_{r}$ be such that we have a direct sum decomposition $\widetilde{M}_{r}=\widetilde{M}_{r-1} \oplus\left\langle\tilde{e}_{r}\right\rangle$. Based on (6) (applied with $t=r)$, we can write $e_{r}=y_{r-1}+l_{r} \tilde{e}_{r}$, where $y_{r-1} \in \widetilde{M}_{r-1}$ and $l_{r} \in W(k) \backslash p^{c_{r}+1} W(k)$. Let $w_{r}:=\sigma\left(l_{r}\right)^{-1}\left(y_{r}-\varphi\left(y_{r-1}\right)\right) \in \widetilde{M}_{r}\left[\frac{1}{p}\right]$. We have $\varphi\left(\tilde{e}_{r}\right)=w_{r}+\sigma\left(l_{r}\right)^{-1} p^{n_{r}} x_{r} \in M$. As $n_{r}>c_{r+1}>c_{r}$, we have $\sigma\left(l_{r}\right)^{-1} p^{n_{r}} x_{r} \in p^{n_{r}-c_{r}} M \subseteq M$; thus $w_{r} \in \widetilde{M}_{r}=\widetilde{M}_{r}\left[\frac{1}{p}\right] \cap M$. Let $\eta_{r}$ be the $\sigma$-linear endomorphism of $\widetilde{M}_{r}$ that acts on $\widetilde{M}_{r-1}$ as $\varphi$ does and that takes $\tilde{e}_{r}$ into $w_{r}$; thus $\eta_{r}\left(e_{r}\right)=y_{r}$. The difference $\varphi\left(\tilde{e}_{r}\right)-\eta_{r}\left(\tilde{e}_{r}\right)$ is $\sigma\left(l_{r}\right)^{-1} p^{n_{r}} x_{r} \in p^{n_{r}-c_{r}} M$. Thus $\varphi$ restricted to $\widetilde{M}_{r}$ and $\eta_{r}$, when viewed as maps from $\widetilde{M}_{r}$ to $M$, coincide $\bmod p^{n_{r}-c_{r}}$. From this and the inequality $n_{r}-c_{r} \geqslant h+1$, we get that the pair $\left(\widetilde{M}_{r}, \eta_{r}\right)$ is an $F$-crystal over $k$ whose $h$-number is at most $h$.

Let $O_{r}$ be a $W(k)$-submodule of $\widetilde{M}_{r}$ such that $p^{\mathbf{T}_{+}\left(\widetilde{M}_{r}, \eta_{r}\right)} \widetilde{M}_{r} \subseteq O_{r}$ and $\left(O_{r}, \eta_{r}\right)$ is a Dieudonné $p$-divisible object over $k$. Let $t_{r} \in S\left(0, \mathbf{T}_{+}\left(\widetilde{M}_{r}, \eta_{r}\right)\right)$ be the smallest number such that $p^{t_{r}} e_{1} \in O_{r} \backslash p O_{r}$. We consider a direct sum decomposition $\left(O_{r}, \eta_{r}\right)=\left(O_{r, 1}, \eta_{r}\right) \oplus \cdots \oplus$ $\left(O_{r, s_{r}}, \eta_{r}\right)$ into elementary Dieudonné $p$-divisible objects over $k$; we can assume that the indices are such that the component $e_{1,1} \in O_{r, 1}$ of $p^{t_{r}} e_{1} \in O_{r}$ with respect to this direct sum decomposition of $O_{r}$, is not divisible inside $O_{r, 1}$ by $p$ (i.e. we have $e_{1,1} \notin p O_{r, 1}$ ). Let $u_{r} \in[0, h]$ be the unique slope of $\left(O_{r, 1}, \eta_{r}\right)$.

The element $p^{r ! a \alpha} e_{1}=\varphi^{r ! a}\left(e_{1}\right)$ is congruent $\bmod p^{n_{r}-c_{r}}$ to $\eta_{r}^{r ! a}\left(e_{1}\right)$. As we have $p^{\mathbf{T}_{+}\left(\widetilde{M}_{r}, \eta_{r}\right)} \widetilde{M}_{r} \subseteq O_{r}$, we get that $\eta_{r}^{r ! a}\left(e_{1,1}\right)-p^{r ! a \alpha} e_{1,1} \in p^{t_{r}+n_{r}-c_{r}-\mathbf{T}_{+}\left(\widetilde{M}_{r}, \eta_{r}\right)} O_{r, 1}$; thus $\eta_{r}^{r ! a}\left(e_{1,1}\right)-p^{r ! a \alpha} e_{1,1} \in p^{n_{r}-c_{r}-\mathbf{T}_{+}\left(\widetilde{M}_{r}, \eta_{r}\right)} O_{r, 1}$. As $\left(O_{r, 1}, \eta_{r}\right)$ is an elementary Dieudonné $p$-divisible object over $k$ whose rank divides $r ! a$ and as $e_{1,1} \in O_{r, 1} \backslash p O_{r, 1}$, there exists $z_{r} \in$ $O_{r, 1} \backslash p O_{r, 1}$ such that $\eta_{r}^{r ! a}\left(e_{1,1}\right)=p^{r ! a u_{r}} z_{r}$. Thus $p^{r ! a u_{r}} z_{r}-p^{r ! a \alpha} e_{1,1} \in p^{n_{r}-c_{r}-\mathbf{T}_{+}\left(\widetilde{M}_{r}, \eta_{r}\right)} O_{r, 1}$. As $d_{r} \geqslant \mathbf{T}_{+}\left(\widetilde{M}_{r}, \eta_{r}\right)$ and $n_{r}>c_{r+1}$, from (8) we get $n_{r}-c_{r}-\mathbf{T}_{+}\left(\widetilde{M}_{r}, \eta_{r}\right) \geqslant r ! h a+1$. As $h \geqslant \max \left\{u_{r}, \alpha\right\}$, we have $r ! a h+1>\max \left\{r ! a u_{r}, r ! a \alpha\right\}$. Thus

$$
n_{r}-c_{r}-\mathbf{T}_{+}\left(\widetilde{M}_{r}, \eta_{r}\right)>\max \left\{r ! a u_{r}, r ! a \alpha\right\} .
$$

From (9) and the relations $p^{r ! a u_{r}} z_{r}-p^{r ! a \alpha} e_{1,1} \in p^{n_{r}-c_{r}-\mathbf{T}_{+}\left(M_{r}, \eta_{r}\right)} O_{r, 1}$ and $z_{r}, e_{1,1} \in O_{r, 1} \backslash$ $p O_{r, 1}$, we get that $r ! a u_{r}=r ! a \alpha$. Thus $\alpha=u_{r}$ and therefore $a=r_{O_{r, 1}} \leqslant r$. This contradicts the fact that $r \in S(1, a-1)$. Thus $n_{r} \leqslant c_{r+1}$ and so (7) holds. Thus (6) holds for $t=r+1$. As $c_{r+1}$ depends only on $r, a, h, c_{r}$, and $d_{r}$ and as (by induction) $c_{r}$ and $d_{r}$ depend only on $r, a$, and $h$, we get that $c_{r+1}$ depends only on $a$ and $c=h$. This ends our second induction on $t \in S(1, a)$. Thus the claim holds.

We have $p^{c_{a}} M=p^{c_{a}} \widetilde{M}_{a} \subseteq M_{a} \subseteq M$, cf. (6). As $\left(M_{a}, \varphi\right)$ is an elementary Dieudonné $p$-divisible object over $k$, we get $\mathbf{T}_{+}(M, \varphi) \leqslant c_{a}$. This ends case 2 . 
The above two cases imply that $\mathbf{T}_{+}(M, \varphi)$ has upper bounds that are effectively computable in terms of $a=r_{M}$ and $c=h$. Thus the number $d_{+}(a, 0, c)$ exists and has effectively computable upper bounds in terms of $a$ and $c$. This ends the initial induction (on ranks $a$ ) and so it also ends the proof of Lemma 2.4.1.

\subsubsection{An interpretation}

The estimates of the proof of 2.4.1.1 are different from the ones of [22, §I]. It seems to us that loc. cit. can be used in order to improve these estimates. Accordingly, we now make the connection between loc. cit. [10, §2], and 2.4.1.1. We situate ourselves in the context of case 2 of 2.4.1. Let $O(\alpha)$ be the $\mathbf{Z}_{p}$-algebra of endomorphisms of the unique elementary Dieudonné $p$-divisible object over $k$ of slope $\alpha$. It is known that $O(\alpha)$ is an order of the central division algebra over $\mathbf{Q}_{p}$ whose invariant is the image of the non-negative rational number $\alpha$ in $\mathbf{Q} / \mathbf{Z}$, cf. [5, Ch. IV, §3]. There exists a $W(k)$-submodule $\widetilde{M}$ of $M$ that contains $p^{-\left[-\left(r_{M}-1\right) \alpha\right]} M$ and such that the pair $(\widetilde{M}, \varphi)$ is an $\alpha$-divisible $F$-crystal over $k$ (i.e. for all $n \in \mathbf{N}$ we can write $\varphi^{n}$ as $p^{[n \alpha]}$ times a $\sigma^{n}$-linear endomorphism $\varphi_{n}$ of $\left.\widetilde{M}\right)$, cf. [22, pp. 151-152]. As we have $r_{M} \alpha \in \mathbf{N} \cup\{0\}$, all slopes of $\left(M, \varphi_{r_{M}}\right)$ are 0 . Thus $\varphi_{r_{M}}(\widetilde{M})=\widetilde{M}$. Triples of the form $\left(\widetilde{M}, \varphi, \varphi_{r_{M}}\right)$ are easily classified. Their isomorphism classes are in one-to-one correspondence to isomorphism classes of torsion free $O(\alpha)$-modules which by inverting $p$ become free $O(\alpha)\left[\frac{1}{p}\right]$-modules of rank 1, see $[10,2.4$ and 2.5]. It is easy to see that under this correspondence, Claim 2.4.1.1 is equivalent to the following well known result.

2.4.2.1. Claim. - There exists a smallest number $N(\alpha) \in \mathbf{N}$ which has effectively computable upper bounds and for which the following property holds: for any element $x \in O(\alpha) \backslash$ $p O(\alpha)$, the length of the artinian $\mathbf{Z}_{p}$-module $O(\alpha) / O(\alpha) x$ is at most $N(\alpha)$.

\subsection{Standard $Z_{p}$ structures}

Let $\left(M, \varphi, G,\left(t_{\alpha}\right)_{\alpha \in \mathcal{J}}\right)$ be a latticed $F$-isocrystal with a group and an emphasized family of tensors over $k$ such that the $W$-condition holds for $(M, \varphi, G)$ (see 2.2.1(b) and (d)). Let $M=\bigoplus_{i=a}^{b} \widetilde{F}^{i}(M),\left(F^{i}(M)\right)_{i \in S(a, b)}$, and $\mu: \mathbf{G}_{m} \rightarrow G$ be as in 2.2.1(d). Each tensor $t_{\alpha} \in$ $\mathcal{T}(M)$ is fixed by both $\mu$ and $\varphi$. Let $\mu_{\text {can }}: \mathbf{G}_{m} \rightarrow \mathbf{G L}_{M}$ be the inverse of the canonical split cocharacter of $\left(M,\left(F^{i}(M)\right)_{i \in S(a, b)}, \varphi\right)$ defined in [42, p. 512]. Let $M=\bigoplus_{i=a}^{b} \widetilde{F}_{\text {can }}^{i}(M)$ be the direct sum decomposition such that the cocharacter $\mu_{\text {can }}$ acts on $\widetilde{F}_{\text {can }}^{i}(M)$ via the $-i$-th power of the identity character of $\mathbf{G}_{m}$. We have $F^{i}(M)=\bigoplus_{j=b}^{i} \widetilde{F}_{\text {can }}^{j}(M)$ for all $i \in S(a, b)$ and $M=\bigoplus_{i=a}^{b} \varphi\left(p^{-i} \widetilde{F}_{\text {can }}^{i}(M)\right)$, cf. loc. cit. The cocharacter $\mu_{\text {can }}$ fixes each $t_{\alpha}$ (cf. the functorial aspects of [42, p. 513]) and so it factors through $G$. As $M=\bigoplus_{i=a}^{b} \varphi\left(p^{-i} \widetilde{F}_{\text {can }}^{i}(M)\right)$, the resulting cocharacter $\mu_{\text {can }}: \mathbf{G}_{m} \rightarrow G$ is also a Hodge cocharacter of $(M, \varphi, G)$ in the sense of 2.2.1(d).

Let $\sigma_{0}:=\varphi \mu(p)$. We have $\sigma_{0}(M)=\varphi\left(\bigoplus_{i=a}^{b} p^{-i} \widetilde{F}^{i}(M)\right)=M$. Thus $\sigma_{0}$ is a $\sigma$-linear automorphism of $M$ and so also of $\mathcal{T}(M)$. For $\alpha \in \mathcal{J}$ we have $\sigma_{0}\left(t_{\alpha}\right)=t_{\alpha}$. Let $M_{\mathbf{z}_{p}}:=$ $\left\{m \in M \mid \sigma_{0}(m)=m\right\}$. We now assume $k=\bar{k}$. So $M_{\mathbf{Z}_{p}}$ is a free $\mathbf{Z}_{p}$-module such that we have $M=M_{\mathbf{z}_{p}} \otimes \mathbf{z}_{p} W(k)$ and $t_{\alpha} \in \mathcal{T}\left(M_{\mathbf{z}_{p}}\right)$ for all $\alpha \in \mathcal{J}$. Let $G_{\mathbf{Q}_{p}}$ be the subgroup of $\mathbf{G L}_{M \mathbf{z}_{p}\left[\frac{1}{p}\right]}$ that fixes $t_{\alpha}$ for all $\alpha \in \mathcal{J}$; its pullback to $\operatorname{Spec}(B(k))$ is $G_{B(k)}$. Let $G_{\mathbf{Z}_{p}}$ be the Zariski closure of $G_{\mathbf{Q}_{p}}$ in $\mathbf{G L}_{M_{\mathbf{z}_{p}}}$. As $G$ is the Zariski closure of $G_{B(k)}$ in $\mathbf{G L}_{M}$, we get that $G$ is the pullback to $\operatorname{Spec}(W(k))$ of $G_{\mathbf{Z}_{p}}$. If moreover we have a principal bilinear quasipolarization $\lambda_{M}: M \otimes_{W(k)} M \rightarrow W(k)$ of $(M, \varphi, G)$, then $\lambda_{M}$ is also the extension to $W(k)$ of a perfect bilinear form $\lambda_{M_{\mathbf{z}_{p}}}$ on $M_{\mathbf{z}_{p}}$.

$4^{\text {e }}$ SÉRIE - TOME $39-2006-\mathrm{N}^{\circ} 2$ 


\subsection{Exponentials}

Let $H=\operatorname{Spec}(A)$ be an integral, affine group scheme of finite type over $\operatorname{Spec}(W(k))$. Let $O$ be a free $W(k)$-module of finite rank such that we have a closed embedding homomorphism $H \hookrightarrow \mathbf{G L}_{O}$; one constructs $O$ as a $W(k)$-submodule of $A$ (cf. [6, vol. I, Exp. $\left.\mathrm{VI}_{B}, 11.11 .1\right]$ ). If $p \geqslant 3$, let $E_{O}:=p \operatorname{End}(O)$. If $p=2$, let $E_{O}$ be the sum of $p^{2} \operatorname{End}(O)$ and of the set of nilpotent elements of $p \operatorname{End}(O)$. Let

$$
\exp : E_{O} \rightarrow \mathbf{G L}_{O}(W(k))
$$

be the exponential map that takes $x \in E_{O}$ into $\sum_{i=0}^{\infty} \frac{x^{i}}{i !}$; here $x^{0}:=1_{O}$.

Let $l \in \mathbf{N}$. Here are the well known properties of the map exp we will often use.

(a) If $p \geqslant 3$ and $x \in p^{l} \operatorname{End}(O)$, then $\exp (x)$ is congruent $\bmod p^{2 l}$ to $1_{O}+x$.

(b) If $p=2, l \geqslant 2$, and $x \in p^{l} \operatorname{End}(O)$, then $\exp (x)$ is congruent $\bmod p^{2 l}$ to $1_{O}+x+\frac{x^{2}}{2}$ and is congruent $\bmod p^{2 l-1}$ to $1_{O}+x$.

(c) If $x \in \operatorname{Lie}\left(H_{B(k)}\right) \cap E_{O}$, then $\exp (x) \in H(W(k))$.

To check (c) it is enough to show that $\exp (x) \in H(B(k))$. It suffices to check this under the extra assumption that the transcendental degree of $k$ is countable. Fixing an embedding $W(k) \hookrightarrow$ $\mathbf{C}$, we can view $H(\mathbf{C})$ as a Lie subgroup of $\mathbf{G} \mathbf{L}_{O}(\mathbf{C})$; so the relation $\exp (x) \in H(B(k))$ follows easily from [20, Ch. II, §1, 3].

2.6.1. LemmA. - Suppose $H$ is smooth over $\operatorname{Spec}(W(k))$. Let $l \in \mathbf{N}$. Let $g_{l} \in H(W(k))$ be congruent $\bmod p^{l}$ to $1_{O}$. Then for any $i \in S(1, l)$ there exists $z_{i, l} \in \operatorname{Lie}(H)$ such that $g_{l}$ is congruent $\bmod p^{i+l}$ to $1_{O}+p^{l} z_{i, l}$.

Proof. - We use induction on $i$. The case $i=1$ is trivial. Let $\bar{z}_{1, l}$ be the reduction $\bmod p$ of $z_{1, l}$. The passage from $i$ to $i+1$ goes as follows. We first consider the case when either $p \geqslant 3$ or $p=2$ and $i+1<l$. Let $g_{l+1}:=g_{l} \exp \left(-p^{l} z_{1, l}\right) \in H(W(k))$; it is congruent $\bmod p^{l+1}$ to $\left(1_{O}+p^{l} z_{1, l}\right)\left(1_{O}-p^{l} z_{1, l}\right)$ (cf. 2.6(a) and (b)) and so also to $1_{O}$. By replacing $g_{l}$ with $g_{l+1}$, the role of the pair $(i+1, l)$ is replaced by the one of the pair $(i, l+1)$. As $1_{O}+p^{l} z_{1, l}$ and $\exp \left(p^{l} z_{1, l}\right)$ are congruent $\bmod p^{i+1+l}$ (cf. 2.6(a) and (b)), by induction we get that $g_{l}=g_{l+1} \exp \left(p^{l} z_{1, l}\right)$ is congruent $\bmod p^{i+1+l}$ to $\left(1_{O}+p^{l+1} z_{i, l+1}\right)\left(1_{O}+p^{l} z_{1, l}\right)$ and so also to $1_{O}+p^{l}\left(z_{1, l}+p z_{i, l+1}\right)$. Thus as $z_{i+1, l}$ we can take the sum $z_{1, l}+p z_{i, l+1}$.

Let now $p=2$ and $i+1=l \geqslant 2$. We have $\bar{z}_{1, l}^{2} \in \operatorname{Lie}\left(H_{k}\right)$, cf. [1, Ch. II, 3.1, 3.5, Lemma 3 of 3.19]. Thus there exists $\tilde{z}_{1, l} \in \operatorname{Lie}(H)$ that is congruent $\bmod 2$ to $z_{1, l}^{2}$. But $1_{O}-2^{l} z_{1, l}$ is congruent mod $2^{2 l}$ to $\exp \left(-2^{l} z_{1, l}\right) \exp \left(-2^{2 l-1} \tilde{z}_{1, l}\right)$, cf. $2.6(\mathrm{~b})$. The existence of $z_{i+1, l}$ is now argued as in the previous paragraph but working with $g_{l+1}:=g_{l} \exp \left(-2^{l} z_{1, l}\right) \exp \left(-2^{2 l-1} \tilde{z}_{1, l}\right) \in$ $H(W(k))$. This ends the induction.

2.6.2. LemmA. - Suppose $H$ is smooth over $\operatorname{Spec}(W(k))$. Let $l \in \mathbf{N}$. If $z_{l} \in \operatorname{Lie}(H)$, then the reduction $\bmod p^{2 l}$ of $1_{O}+p^{l} z_{l}$ belongs to $H\left(W_{2 l}(k)\right)$.

Proof. - We can assume $p=2$ (cf. 2.6(a) and (c) applied with $x=p^{l} z_{l}$ ) and $l \geqslant 2$ (as $H$ is smooth). By replacing $1_{O}+2^{l} z_{l}$ with $\left(1_{O}+2^{l} z_{l}\right) \exp \left(-2^{l} z_{l}\right)$, we can assume (cf. 2.6(b)) that $z_{l} \in 2^{l-1} \operatorname{Lie}(H)$. But this case is obvious (as $H$ is smooth).

\subsection{Dilatations}

In this section we study an arbitrary integral, closed subgroup scheme $G=\operatorname{Spec}\left(R_{G}\right)$ of $\mathbf{G L}_{M}$. Let $W(k)^{\text {sh }}$ be the strict henselization of $W(k)$. If $a: \operatorname{Spec}\left(W(k)^{\mathrm{sh}}\right) \rightarrow G$ is a morphism, then the Néron measure of the defect of smoothness $\delta(a) \in \mathbf{N} \cup\{0\}$ of $G$ at $a$ is the length of the torsion part of the coherent $\mathcal{O}_{\operatorname{Spec}\left(W(k)^{\text {sh }}\right)}$-module $a^{*}\left(\Omega_{G / \operatorname{Spec}(W(k))}\right)$. Here $\Omega_{G / \operatorname{Spec}(W(k))}$ 
is the coherent $\mathcal{O}_{G}$-module of relative differentials of $G$ with respect to $\operatorname{Spec}(W(k))$. As $G$ is a group scheme, the value of $\delta(a)$ does not depend on $a$ and so we denote it by $\delta(G)$. We have $\delta(G) \in \mathbf{N}$ if and only if $G$ is not smooth over $\operatorname{Spec}(W(k))$, cf. [2, 3.3, Lemma 1]. Let $S(G)$ be the Zariski closure in $G_{k}$ of all special fibres of $W(k)^{\text {sh }}$-valued points of $G$. It is a reduced subgroup of $G_{k}$. We write $S(G)=\operatorname{Spec}\left(R_{G} / J_{G}\right)$, where $J_{G}$ is the ideal of $R_{G}$ that defines $S(G)$.

By the canonical dilatation of $G$ we mean the affine $G$-scheme $G_{1}=\operatorname{Spec}\left(R_{G_{1}}\right)$, where $R_{G_{1}}$ is the $R_{G}$-subalgebra of $R_{G}\left[\frac{1}{p}\right]$ generated by $\frac{x}{p}$, with $x \in J_{G}$. The $\operatorname{Spec}(W(k))$-scheme $G_{1}$ is integral and has a canonical group scheme structure with respect to which the morphism $G_{1} \rightarrow G$ is a homomorphism of group schemes over $\operatorname{Spec}(W(k))$, cf. [2, 3.2, p. 63 and (d) of p. 64]. The morphism $G_{1} \rightarrow G$ has the following universal property (cf. [2, 3.2, Prop. 1]): any morphism $Y \rightarrow G$ of flat $\operatorname{Spec}(W(k))$-schemes whose special fibre factors through the closed embedding $S(G) \hookrightarrow G_{k}$, factors uniquely through $G_{1} \rightarrow G$. Either $\delta\left(G_{1}\right)=0$ (i.e. $G_{1}$ is smooth over $\operatorname{Spec}(W(k)))$ or (cf. [2, 3.3, Prop. 5]) we have $0<\delta\left(G_{1}\right)<\delta(G)$.

By using at most $\delta(G)$ canonical dilatations, we get the existence of a unique smooth, affine group scheme $G^{\prime}$ over $\operatorname{Spec}(W(k))$ that is endowed with a homomorphism $G^{\prime} \rightarrow G$ whose fibre over $\operatorname{Spec}(B(k))$ is an isomorphism and that has the following universal property (cf. [2, 7.1, Thm. 5]): any morphism $Y \rightarrow G$ of $\operatorname{Spec}(W(k))$-schemes with $Y$ smooth, factors uniquely through $G^{\prime} \rightarrow G$. In particular, we can identify $G^{\prime}\left(W(k)^{\text {sh }}\right)$ with $G\left(W(k)^{\text {sh }}\right)$. The homomorphism $G^{\prime} \rightarrow G$ is called the group smoothening of $G$. Let

$$
n(G) \in S(0, \delta(G))
$$

be the smallest number of canonical dilatations one has to perform in order to construct $G^{\prime}$. We have $n(G)=0$ if and only if $G$ is smooth over $\operatorname{Spec}(W(k))$.

The closed embedding $i_{G}: G \hookrightarrow \mathbf{G L}_{M}$ gets replaced by a canonical homomorphism $i_{G^{\prime}}: G^{\prime} \rightarrow \mathbf{G L}_{M}$ that factors through $i_{G}$. We identify $\operatorname{Lie}\left(G^{\prime}\right)$ with a $W(k)$-lattice of $\operatorname{Lie}\left(G_{B(k)}\right)$ contained in $\operatorname{End}(M)$. Let $d_{\mathrm{sm}} \in \mathbf{N} \cup\{0\}$ be the smallest number such that we have $p^{d_{\mathrm{sm}}}\left(\operatorname{Lie}\left(G_{B(k)}\right) \cap \operatorname{End}(M)\right) \subseteq \operatorname{Lie}\left(G^{\prime}\right) \subseteq \operatorname{Lie}\left(G_{B(k)}\right) \cap \operatorname{End}(M)$.

We fix a closed embedding homomorphism $G^{\prime} \hookrightarrow \mathbf{G L}_{M^{\prime}}$, where $M^{\prime}$ is a free $W(k)$-module of finite rank (see beginning of 2.6). Let $g \in G^{\prime}(W(k))=G(W(k))$.

2.7.1. Definition. - Let $n \in \mathbf{N}$. We say $g$ is congruent $\bmod p^{n}$ to $1_{M^{\prime}}$ (respectively to $1_{M}$ ) if and only if the image of $g$ in $G^{\prime}\left(W_{n}(k)\right)$ (respectively in $G\left(W_{n}(k)\right)$ ) is the identity element.

2.7.2. LEMMA. - We have the following three properties:

(a) If $g$ is congruent $\bmod p^{n}$ to $1_{M^{\prime}}$, then $g$ is also congruent $\bmod p^{n}$ to $1_{M}$.

(b) If $g$ is congruent $\bmod p^{n+n(G)}$ to $1_{M}$, then $g$ is also congruent mod $p^{n}$ to $1_{M^{\prime}}$.

(c) We have an inequality $d_{\mathrm{sm}} \leqslant n(G)$.

Proof. - Part (a) is trivial. We write $G^{\prime}=\operatorname{Spec}\left(R_{G^{\prime}}\right)$ and $\mathbf{G L}_{M}=\operatorname{Spec}\left(R_{M}\right)$. Let $I_{G}, I_{G_{1}}$, $I_{G^{\prime}}$, and $I_{M}$ be the ideals of $R_{G}, R_{G_{1}}, R_{G^{\prime}}$, and $R_{M}$ (respectively) that define the identity sections. We have $I_{G_{1}}=I_{G}\left[\frac{1}{p}\right] \cap R_{G_{1}}$ and $I_{G^{\prime}}=I_{G}\left[\frac{1}{p}\right] \cap R_{G^{\prime}}$.

We check (b). Let $m_{g}: R_{G} \rightarrow W(k)$ be the homomorphism that defines $g$; we have $m_{g}\left(I_{G}\right) \subseteq p^{n+n(G)} W(k)$. Let $m_{1 g}: R_{G_{1}} \rightarrow W(k)$ be the homomorphism through which $m_{g}$ factors. We have $m_{1 g}\left(I_{G_{1}}\right) \subseteq p^{n+n(G)-1} W(k)$, cf. the very definition of $R_{G_{1}}$. Part (b) follows from a repeated application of this fact to the sequence of $n(G)$ dilatations performed to construct $G^{\prime}$. The cokernel of the cotangent map (computed at $W(k)$-valued identity elements) $I_{G} / I_{G}^{2} \rightarrow I_{G_{1}} / I_{G_{1}}^{2}$ is annihilated by $p$, cf. the very definition of $R_{G_{1}}$. By applying this repeatedly, we get that the cokernel of the cotangent map $I_{M} / I_{M}^{2} \rightarrow I_{G^{\prime}} / I_{G^{\prime}}^{2}$ is annihilated 
by $p^{n(G)}$. Taking duals we get that the cokernel of the $W(k)$-linear Lie monomorphism $\operatorname{Lie}\left(G^{\prime}\right) \hookrightarrow \operatorname{Lie}\left(G_{B(k)}\right) \cap \operatorname{Lie}\left(\mathbf{G L}_{M}\right)$ is also annihilated by $p^{n(G)}$. As $\operatorname{Lie}\left(\mathbf{G L}_{M}\right)$ is the Lie algebra associated to $\operatorname{End}(M)$, we get that (c) holds.

\subsection{Complements on $\mathcal{M}\left(W_{q}(S)\right)$}

Let $q \in \mathbf{N}$ and let $l \in S(0, q)$. Let $f: S_{1} \rightarrow S$ be a morphism of $\operatorname{Spec}\left(\mathbf{F}_{p}\right)$-schemes. Let $f_{q}: W_{q}\left(S_{1}\right) \rightarrow W_{q}(S)$ be the natural morphism of $\operatorname{Spec}\left(\mathbf{Z} / p^{q} \mathbf{Z}\right)$-schemes defined by $f$. Let $\mathfrak{C}$ be an $F$-crystal over $S$. In this section we include four complements on the category $\mathcal{M}\left(W_{q}(S)\right)$.

\subsubsection{Pullbacks}

Let $f_{q}^{*}: \mathcal{M}\left(W_{q}(S)\right) \rightarrow \mathcal{M}\left(W_{q}\left(S_{1}\right)\right)$ be the natural pullback functor. So if $S=\operatorname{Spec}(R)$ and $S_{1}=\operatorname{Spec}\left(R_{1}\right)$ are affine and if $h:\left(O, \varphi_{O}\right) \rightarrow\left(O^{\prime}, \varphi_{O^{\prime}}\right)$ is a morphism of $\mathcal{M}\left(W_{q}(S)\right)$, then $f_{q}^{*}(h)$ is the morphism

$$
h \otimes 1_{W_{q}\left(R_{1}\right)}:\left(O \otimes_{W_{q}(R)} W_{q}\left(R_{1}\right), \varphi_{O} \otimes \Phi_{R_{1}}\right) \rightarrow\left(O^{\prime} \otimes_{W_{q}(R)} W_{q}\left(R_{1}\right), \varphi_{O^{\prime}} \otimes \Phi_{R_{1}}\right) .
$$

In general $W_{q}(S) \times_{W_{q+1}(S)} W_{q+1}\left(S_{1}\right)$ is not $W_{q}\left(S_{1}\right)$. Thus, in general the restriction of $f_{q+1}^{*}$ to $\mathcal{M}\left(W_{q}(S)\right)$ and $f_{q}^{*}$ do not coincide as functors from $\mathcal{M}\left(W_{q}(S)\right)$ to $\mathcal{M}\left(W_{q}\left(S_{1}\right)\right)$ and therefore the sequence of functors $\left(f_{q}^{*}\right)_{q \in \mathbf{N}}$ does not define a pullback functor from $\mathcal{M}(W(S))$ to $\mathcal{M}\left(W\left(S_{1}\right)\right)$. If the Frobenius endomorphism of $\mathcal{O}_{S_{1}}$ is surjective, then regardless of what $S$ is we have $W_{q}(S) \times_{W_{q+1}(S)} W_{q+1}\left(S_{1}\right)=W_{q}\left(S_{1}\right)$ and thus the sequence of functors $\left(f_{q}^{*}\right)_{q \in \mathbf{N}}$ does define a pullback functor $f^{*}: \mathcal{M}(W(S)) \rightarrow \mathcal{M}\left(W\left(S_{1}\right)\right)$.

If $u$ is an object (or a morphism) of $\mathcal{M}\left(W_{q}(S)\right)$, then by its pullback to an object (or a morphism) of $\mathcal{M}\left(W_{q}\left(S_{1}\right)\right)$ we mean $f_{q}^{*}(u)$. If $t \in \mathbf{N}$ and if $f_{q+t}^{*}(u)$ is an object (or a morphism) of $\mathcal{M}\left(W_{q}\left(S_{1}\right)\right)$, then we have $f_{q+t}^{*}(u)=f_{q}^{*}(u)$. If $S_{1}$ is the spectrum of a perfect field, we also speak simply of the pullback of $u$ via $f$, to be often denoted as $f^{*}(u)$ (instead of either $f_{q}^{*}(u)$ or $\left.f_{q+t}^{*}(u)\right)$.

If $S$ is integral, if $k_{S}$ is the field of fractions of $S$, and if $u$ is a morphism of $\mathcal{M}\left(W_{q}(S)\right)$, then we say $\operatorname{Coker}(u)$ is generically annihilated by $p^{l}$ if the pullback of $u$ to a morphism of $\mathcal{M}\left(W_{q}\left(k_{S}\right)\right)=\mathcal{M}\left(W_{q}\left(\operatorname{Spec}\left(k_{S}\right)\right)\right)$ has a cokernel annihilated by $p^{l}$.

\subsubsection{The evaluation functor $E$}

Let $\delta_{q}(S)$ be the canonical divided power structure of the ideal sheaf of $\mathcal{O}_{W_{q}(S)}$ that defines the closed embedding $S \hookrightarrow W_{q}(S)$. The evaluation of the $F$-crystal $\mathfrak{C}$ at the thickening $(S \hookrightarrow$ $\left.W_{q}(S), \delta_{q}(S)\right)$ is a triple $\left(\mathcal{F}_{q}, \varphi_{\mathcal{F}_{q}}, \nabla_{\mathcal{F}_{q}}\right)$, where $\mathcal{F}_{q}$ is a locally free $\mathcal{O}_{W_{q}(S)}$-module of finite rank, where $\varphi_{\mathcal{F}_{q}}: \mathcal{F}_{q} \rightarrow \mathcal{F}_{q}$ is a $\Phi_{S}$-linear endomorphism, and where $\nabla_{\mathcal{F}_{q}}$ is an integrable and topologically nilpotent connection on $\mathcal{F}_{q}$, that satisfies certain axioms. In this paper, connections as $\nabla_{\mathcal{F}_{q}}$ will play no role; on the other hand, we will often use the following object

$$
\mathbf{E}\left(\mathfrak{C} ; W_{q}(S)\right):=\left(\mathcal{F}_{q}, \varphi_{\mathcal{F}_{q}}\right)
$$

of $\mathcal{M}\left(W_{q}(S)\right)$. A morphism $v: \mathfrak{C} \rightarrow \mathfrak{C}_{1}$ of $F$-crystals over $S$ defines naturally a morphism

$$
\mathbf{E}\left(v ; W_{q}(S)\right): \mathbf{E}\left(\mathfrak{C} ; W_{q}(S)\right) \rightarrow \mathbf{E}\left(\mathfrak{C}_{1} ; W_{q}(S)\right) .
$$

The association $v \rightarrow \mathbf{E}\left(v ; W_{q}(S)\right)$ defines a $\mathbf{Z}_{p}$-linear (evaluation) functor from the category of $F$-crystals over $S$ into the category $\mathcal{M}\left(W_{q}(S)\right)$.

To ease notations, let $\mathbf{E}\left(\mathfrak{C} ; W_{q}\left(S_{1}\right)\right):=\mathbf{E}\left(\mathfrak{C}_{S_{1}} ; W_{q}\left(S_{1}\right)\right)$ and, in the case when $S_{1}=$ $\operatorname{Spec}\left(R_{1}\right)$ is affine, let $\mathbf{E}\left(\mathfrak{C} ; W_{q}\left(R_{1}\right)\right):=\mathbf{E}\left(\mathfrak{C} ; W_{q}\left(S_{1}\right)\right)$. 
The functorial morphism $f_{q}:\left(S_{1} \hookrightarrow W_{q}\left(S_{1}\right), \delta_{q}\left(S_{1}\right)\right) \rightarrow\left(S \hookrightarrow W_{q}(S), \delta_{q}(S)\right)$ gives birth to a canonical isomorphism (to be viewed as an identity)

$$
c_{f ; q}: f_{q}^{*}\left(\mathbf{E}\left(\mathfrak{C} ; W_{q}(S)\right)\right) \stackrel{\sim}{\sim} \mathbf{E}\left(\mathfrak{C} ; W_{q}\left(S_{1}\right)\right) .
$$

If $e: S_{2} \rightarrow S_{1}$ is another morphism of $\operatorname{Spec}\left(\mathbf{F}_{p}\right)$-schemes, then we have identities

$$
(f \circ e)_{q}^{*}=e_{q}^{*} \circ f_{q}^{*} \quad \text { and } \quad c_{e ; q} \circ e_{q}^{*}\left(c_{f ; q}\right)=c_{f \circ e ; q} .
$$

In what follows we will use without any extra comment the identities (10) and (11).

\subsubsection{Inductive limits}

Let $V \hookrightarrow V_{1}$ be a monomorphism of commutative $\mathbf{F}_{p}$-algebras. Suppose we have an inductive limit $V_{1}=$ ind $\lim _{\alpha \in \Lambda} V_{\alpha}$ of commutative $V$-subalgebras of $V_{1}$ indexed by the set of objects $\Lambda$ of a filtered, small category. For $\alpha \in \Lambda$, let $f^{\alpha}: \operatorname{Spec}\left(V_{\alpha}\right) \rightarrow \operatorname{Spec}(V)$ be the natural morphism.

Let $\left(O, \varphi_{O}\right)$ and $\left(O^{\prime}, \varphi_{O^{\prime}}\right)$ be objects of $\mathcal{M}\left(W_{q}(V)\right)$ such that $O$ and $O^{\prime}$ are free $W_{q}(V)$ modules of finite rank. Let $\left(O_{1}, \varphi_{O_{1}}\right)$ and $\left(O_{1}^{\prime}, \varphi_{O_{1}^{\prime}}\right)$ be the pullbacks of $\left(O, \varphi_{O}\right)$ and $\left(O^{\prime}, \varphi_{O^{\prime}}\right)$ (respectively) to objects of $\mathcal{M}\left(W_{q}\left(V_{1}\right)\right)$. We consider a morphism

$$
u_{1}:\left(O_{1}, \varphi_{O_{1}}\right) \rightarrow\left(O_{1}^{\prime}, \varphi_{O_{1}^{\prime}}\right)
$$

of $\mathcal{M}\left(W_{q}\left(V_{1}\right)\right)$ whose cokernel is annihilated by $p^{l}$. We fix ordered $W_{q}(V)$-bases $\mathcal{B}_{O}$ and $\mathcal{B}_{O^{\prime}}$ of $O$ and $O^{\prime}$ (respectively). Let $B_{1}$ be the matrix representation of $u_{1}$ with respect to the ordered $W_{q}\left(V_{1}\right)$-basis of $O_{1}$ and $O_{1}^{\prime}$ defined naturally by $\mathcal{B}_{O}$ and $\mathcal{B}_{O^{\prime}}$ (respectively). As $p^{l} \operatorname{Coker}\left(u_{1}\right)=0$, for $x^{\prime} \in \mathcal{B}_{O^{\prime}}$ we can write $p^{l} x^{\prime} \otimes 1=u_{1}\left(\sum_{x \in \mathcal{B}_{O}} x \otimes \beta_{x x^{\prime}}\right)$, where each $\beta_{x x^{\prime}} \in W_{q}\left(V_{1}\right)$. Let $V_{u_{1}}$ be the $V$-subalgebra of $V_{1}$ generated by the components of the Witt vectors of length $q$ with coefficients in $V_{1}$ that are either entries of $B_{1}$ or $\beta_{x x^{\prime}}$ for some pair $\left(x, x^{\prime}\right) \in \mathcal{B}_{O} \times \mathcal{B}_{O^{\prime}}$. As $V_{u_{1}}$ is a finitely generated $V$-algebra, there exists $\alpha_{0} \in \Lambda$ such that $V_{u_{1}} \hookrightarrow V_{\alpha_{0}}$. This implies that $u_{1}$ is the pullback of a morphism

$$
u_{\alpha_{0}}: f_{q}^{\alpha_{0} *}\left(O, \varphi_{O}\right) \rightarrow f_{q}^{\alpha_{0} *}\left(O^{\prime}, \varphi_{O^{\prime}}\right)
$$

of $\mathcal{M}\left(W_{q}\left(V_{\alpha_{0}}\right)\right)$ whose cokernel is annihilated by $p^{l}$. Here are four special cases of interest.

(a) If $V$ is a field and $V_{1}$ is an algebraic closure of $V$, then as $V_{\alpha}$ 's we can take the finite field extensions of $V$ that are contained in $V_{1}$.

(b) If $V_{1}$ is a local ring of an integral domain $V$, then as $V_{\alpha}$ 's we can take the $V$-algebras of global functions of open, affine subschemes of $\operatorname{Spec}(V)$ that contain $\operatorname{Spec}\left(V_{1}\right)$.

(c) We consider the case when $V$ is a discrete valuation ring that is an $N-2$ ring in the sense of [29, (31.A)], when $V_{1}$ is a faithfully flat $V$-algebra that is also a discrete valuation ring, and when each $V_{\alpha}$ is a $V$-algebra of finite type. The flat morphism $f^{\alpha_{0}}: \operatorname{Spec}\left(V_{\alpha_{0}}\right) \rightarrow \operatorname{Spec}(V)$ has quasi-sections, cf. [18, Ch. IV, Cor. (17.16.2)]. In other words, there exist a finite field extension $\tilde{k}$ of $k$ and a $V$-subalgebra $\widetilde{V}$ of $\tilde{k}$ such that: (i) $\widetilde{V}$ is a local, faithfully flat $V$-algebra of finite type whose field of fractions is $\tilde{k}$, and (ii) we have a morphism $\tilde{f}^{\alpha_{0}}: \operatorname{Spec}(\widetilde{V}) \rightarrow \operatorname{Spec}\left(V_{\alpha_{0}}\right)$ such that $\tilde{f}:=f^{\alpha_{0}} \circ \tilde{f}^{\alpha_{0}}$ is the natural morphism $\operatorname{Spec}(\widetilde{V}) \rightarrow \operatorname{Spec}(V)$. As $V$ is an $N-2$ ring, its normalization in $\tilde{k}$ is a finite $V$-algebra and so a Dedekind domain. This implies that we can assume $\widetilde{V}$ is a discrete valuation ring. For future use, we recall that any excellent ring is a Nagata ring (cf. [29, (34.A)]) and so also an $N-2$ ring (cf. [29, (31.A)]). Let

$$
\tilde{u}: \tilde{f}_{q}^{*}\left(O, \varphi_{O}\right)=\tilde{f}_{q}^{\alpha_{0} *}\left(f_{q}^{\alpha_{0} *}\left(O, \varphi_{O}\right)\right) \rightarrow \tilde{f}_{q}^{*}\left(O^{\prime}, \varphi_{O^{\prime}}\right)=\tilde{f}_{q}^{\alpha_{0} *}\left(f_{q}^{\alpha_{0} *}\left(O^{\prime}, \varphi_{O^{\prime}}\right)\right)
$$

$4^{\text {e }}$ SÉRIE - TOME $39-2006-\mathrm{N}^{\circ} 2$ 
be the pullback of $u_{\alpha_{0}}$ to a morphism of $\mathcal{M}\left(W_{q}(\widetilde{V})\right)$; its cokernel is annihilated by $p^{l}$.

If $V$ is the local ring of an integral $\operatorname{Spec}\left(\mathbf{F}_{p}\right)$-scheme $U$, then $\widetilde{V}$ is a local ring of the normalization of $U$ in $\tilde{k}$. So from (b) we get that there exists an open subscheme $\widetilde{U}$ of this last normalization that has $\widetilde{V}$ as a local ring and that has the property that $\tilde{u}$ extends to a morphism of $\mathcal{M}\left(W_{q}(\widetilde{U})\right)$ whose cokernel is annihilated by $p^{l}$.

(d) If $V$ is reduced and $V_{1}=V^{\text {perf }}$, we can take $\Lambda=\mathbf{N}$ and $V_{n}=V^{\left(p^{n}\right)}(n \in \mathbf{N})$.

\subsubsection{Hom schemes}

Let $\mathcal{O}_{1}$ and $\mathcal{O}_{2}$ be two objects of $\mathcal{M}\left(W_{q}(S)\right)$ such that their underlying $\mathcal{O}_{W_{q}}(S)$-modules are locally free of finite ranks. We consider the functor

$$
\operatorname{Hom}\left(\mathcal{O}_{1}, \mathcal{O}_{2}\right): \operatorname{Sch}^{S} \rightarrow \mathrm{SET}
$$

from the category $\operatorname{Sch}^{S}$ of $S$-schemes to the category SET of sets, with the property that $\operatorname{Hom}\left(\mathcal{O}_{1}, \mathcal{O}_{2}\right)\left(S_{1}\right)$ is the set underlying the $\mathbf{Z} / p^{q} \mathbf{Z}$-module of morphisms of $\mathcal{M}\left(W_{q}\left(S_{1}\right)\right)$ that are between $f_{q}^{*}\left(\mathcal{O}_{1}\right)$ and $f_{q}^{*}\left(\mathcal{O}_{2}\right)$; here $f: S_{1} \rightarrow S$ is as in the beginning of 2.8 .

2.8.4.1. LEMMA. - The functor $\operatorname{Hom}\left(\mathcal{O}_{1}, \mathcal{O}_{2}\right)$ is representable by an affine $S$-scheme which locally is of finite presentation.

Proof. - Localizing, we can assume that $S=\operatorname{Spec}(R)$ is affine and that $\mathcal{O}_{1}=\left(O_{1}, \varphi_{O_{1}}\right)$ and $\mathcal{O}_{2}=\left(O_{2}, \varphi_{O_{2}}\right)$ are such that $O_{1}$ and $O_{2}$ are free $W_{q}(R)$-modules. For $i \in\{1,2\}$ let $r_{i}$ be the rank of $O_{i}$. Let $\operatorname{Hom}\left(O_{1}, O_{2}\right)$ be the affine space (of relative dimension $\left.q r_{1} r_{2}\right)$ over $\operatorname{Spec}(R)$ with the property that for any commutative $R$-algebra $R_{1}, \operatorname{Hom}\left(O_{1}, O_{2}\right)\left(R_{1}\right)$ is the set of $W_{q}\left(R_{1}\right)$-linear maps $x: O_{1} \otimes_{W_{q}(R)} W_{q}\left(R_{1}\right) \rightarrow O_{2} \otimes_{W_{q}(R)} W_{q}\left(R_{1}\right)$. We have an identity $\left(\varphi_{O_{2}} \otimes \Phi_{R_{1}}\right) \circ x=x \circ\left(\varphi_{O_{1}} \otimes \Phi_{R_{1}}\right)$ if and only if $x$ belongs to the subset of $\operatorname{Hom}\left(O_{1}, O_{2}\right)\left(R_{1}\right)$ that is naturally identified with $\operatorname{Hom}\left(\mathcal{O}_{1}, \mathcal{O}_{2}\right)\left(\operatorname{Spec}\left(R_{1}\right)\right)$. As the relation $\left(\varphi_{O_{2}} \otimes \Phi_{R_{1}}\right) \circ x=x \circ\left(\varphi_{O_{1}} \otimes \Phi_{R_{1}}\right)$ defines a closed subscheme of $\operatorname{Hom}\left(O_{1}, O_{2}\right)$ that is of finite presentation, the lemma follows.

\subsection{On two results of commutative algebra}

In 5.4 and Section 6 we will use the following two geometric variations of well known results of commutative algebra.

2.9.1. Lemma. - Let $X$ and $Y$ be two integral, normal, locally noetherian schemes. Let $u: X \rightarrow Y$ be an affine morphism that is birational; let $K$ be the field of fractions of either $X$ or $Y$. Let $D(X)$ and $D(Y)$ be the sets of local rings of $X$ and $Y$ (respectively) that are discrete valuation rings (of $K$ ). If $D(Y) \subseteq D(X)$, then $u$ is an isomorphism.

Proof. - Working locally in the Zariski topology of $Y$, we can assume $X=\operatorname{Spec}\left(R_{X}\right)$ and $Y=\operatorname{Spec}\left(R_{Y}\right)$ are also affine and noetherian. Thus (inside $K$ ) we have

$$
R_{Y} \hookrightarrow R_{X}=\bigcap_{V \in D(X)} V \hookrightarrow \bigcap_{V \in D(Y)} V=R_{Y}
$$

(cf. [29, (17.H)] for the two identities). So $R_{Y}=R_{X}$. Thus $u$ is an isomorphism.

2.9.2. LemMA. - Let $X^{\prime}=\operatorname{Spec}\left(R^{\prime}\right) \rightarrow X=\operatorname{Spec}(R)$ be a morphism between affine schemes which at the level of rings is defined by an integral (i.e. an ind-finite) monomorphism $R \hookrightarrow R^{\prime}$. Then an open subscheme $U$ of $X$ is affine if and only if its pullback $U^{\prime}:=U \times_{X} X^{\prime}$ to $X^{\prime}$ is affine. 
Proof. - It is enough to show that $U$ is affine if $U^{\prime}$ is affine. The morphism $U^{\prime} \rightarrow U$ is surjective (see [29, (5.E)]). So as $U^{\prime}$ is quasi-compact (being affine), $U$ is also quasi-compact. Thus $X^{\text {top }} \backslash U^{\text {top }}$ is the zero locus in $X^{\text {top }}$ of a finite number of elements of $R$. So there exists a finitely generated $\mathbf{Z}$-subalgebra $R_{0}$ of $R$ such that $U$ is the pullback of an open subscheme $U_{0}$ of $\operatorname{Spec}\left(R_{0}\right)$ through the natural morphism $\operatorname{Spec}(R) \rightarrow \operatorname{Spec}\left(R_{0}\right)$.

Let $\Lambda$ (respectively $\Lambda^{\prime}$ ) be the set of finite subsets of $R$ (respectively of $R^{\prime}$ ). For $\alpha \in \Lambda$ (respectively $\alpha^{\prime} \in \Lambda^{\prime}$ ), let $R_{\alpha}$ (respectively $R_{\alpha^{\prime}}^{\prime}$ ) be the $R_{0}$-subalgebra of $R$ (respectively of $R^{\prime}$ ) generated by $\alpha$ (respectively by $\left.\alpha^{\prime}\right)$. Let $X_{\alpha}:=\operatorname{Spec}\left(R_{\alpha}\right)$ and $X_{\alpha^{\prime}}^{\prime}:=\operatorname{Spec}\left(R_{\alpha^{\prime}}^{\prime}\right)$. Let $U_{\alpha}$ and $U_{\alpha^{\prime}}^{\prime}$ be the pullbacks of $U_{0}$ to $X_{\alpha}$ and $X_{\alpha^{\prime}}^{\prime}$ (respectively). As $U_{\alpha^{\prime}}^{\prime}$ is a quasi-compact, open subscheme of $X_{\alpha^{\prime}}^{\prime}$, it is an $X_{\alpha^{\prime}}^{\prime}$-scheme of finite presentation. As the scheme $U^{\prime}$ is affine, by applying [18, Ch. IV, (8.10.5)] to the projective limit $U^{\prime} \hookrightarrow X^{\prime}=\operatorname{proj} \lim _{\alpha^{\prime} \in \Lambda^{\prime}} U_{\alpha^{\prime}}^{\prime} \hookrightarrow X_{\alpha^{\prime}}^{\prime}$ of open embeddings of finite presentation, we get that there exists $\beta^{\prime} \in \Lambda^{\prime}$ such that $U_{\beta^{\prime}}^{\prime}$ is affine. Let $\beta \in \Lambda$ be such that $R_{\beta^{\prime} \cup \beta}^{\prime}$ is a finite $R_{\beta}$-algebra. As $U_{\beta^{\prime} \cup \beta}^{\prime}=U_{\beta^{\prime}}^{\prime} \times_{X_{\beta^{\prime}}^{\prime}} X_{\beta^{\prime} \cup \beta}^{\prime}$ is affine, the scheme $U_{\beta}$ is also affine (cf. Chevalley theorem of [16, Ch. II, (6.7.1)] applied to the finite, surjective morphism $U_{\beta^{\prime} \cup \beta}^{\prime} \rightarrow U_{\beta}$ ). Thus $U=U_{\beta} \times_{X_{\beta}} X$ is affine.

\section{Proof of Main Theorem A and complements}

In 3.1 we prove Main Theorem A stated in 1.2. See formula (18) of 3.1.3 for a concrete expression of the number $n_{\text {fam }}$ mentioned in 1.2. In 3.2 we include interpretations and variants of 1.2 in terms of reductions modulo powers of $p$; in particular, see 3.2.4 for the passage from 1.2 to 1.3. See 3.3 for improvements of the estimates of 3.1.1 to 3.1 .5 in many particular cases of interest. If $p \geqslant 3$ let $\varepsilon_{p}:=1$. Let $\varepsilon_{2}:=2$.

\subsection{Proof of 1.2}

We start the proof of 1.2. Until 3.1.4 we will assume $k=\bar{k}$. Let $v:=\operatorname{dim}\left(G_{B(k)}\right)$. It suffices to prove 1.2 under the extra hypothesis $v \geqslant 1$. Let $\delta(G), n(G) \in \mathbf{N} \cup\{0\}$, the group smoothening $G^{\prime} \rightarrow G$ of $G$, and the closed embedding homomorphism $G^{\prime} \hookrightarrow \mathbf{G L}_{M^{\prime}}$ be as in 2.7. We have $G^{\prime}(W(k))=G(W(k))$.

Let $m:=\mathbf{T}\left(\operatorname{Lie}\left(G^{\prime}\right), \varphi\right) \in \mathbf{N} \cup\{0\}$. Based on Definitions 2.2.2(a) and (b), there exists a $B(k)$-basis $\mathcal{B}=\left\{e_{1}, \ldots, e_{v}\right\}$ of $\operatorname{Lie}\left(G_{B(k)}\right)$ formed by elements of $\operatorname{Lie}\left(G^{\prime}\right)$ and there exists a permutation $\pi$ of $S(1, v)$, such that the following three things hold:

(a) denoting $E:=\left\langle e_{1}, \ldots, e_{v}\right\rangle$, we have $p^{m} \operatorname{Lie}\left(G^{\prime}\right) \subseteq E \subseteq \operatorname{Lie}\left(G^{\prime}\right)$;

(b) if $l \in S(1, v)$, then we have $\varphi\left(e_{l}\right)=p^{n_{l}} e_{\pi(l)}$ for some $n_{l} \in \mathbf{Z}$;

(c) for any cycle $\pi_{0}=\left(l_{1}, \ldots, l_{q}\right)$ of $\pi$, the integers $n_{l_{1}}, \ldots, n_{l_{q}}$ are either all non-negative or all non-positive.

If we have $n_{l_{j}} \geqslant 0$ for all $j \in S(1, q)$, let $\tau\left(\pi_{0}\right):=1$. If there exists $j \in S(1, q)$ such that $n_{l_{j}}<0$, let $\tau\left(\pi_{0}\right):=-1$. Let $n \in \mathbf{N}$ be such that

$$
n \geqslant 2 m+\varepsilon_{p}+n(G) .
$$

Let $g_{n} \in G(W(k))$ be congruent $\bmod p^{n}$ to $1_{M}$. So $g_{n} \in G^{\prime}(W(k))$ is congruent $\bmod p^{n-n(G)}$ to $1_{M^{\prime}}$ (cf. 2.7.2(b)) and below we will only use this congruence.

3.1.1. Claim. - For any $t \in \mathbf{N}$ there exists $\tilde{g}_{t} \in G^{\prime}(W(k))=G(W(k))$ congruent $\bmod p^{n-n(G)+t-1-m}$ to $1_{M^{\prime}}$ and such that $\tilde{g}_{t} g_{n} \varphi \tilde{g}_{t}^{-1} \varphi^{-1} \in G^{\prime}(W(k))=G(W(k))$ is congruent $\bmod p^{n-n(G)+t}$ to $1_{M^{\prime}}$. Thus there exists $\tilde{g}_{0} \in G^{\prime}(W(k))=G(W(k))$ congruent $\bmod p^{n-n(G)-m}$ to $1_{M^{\prime}}$ and such that $\tilde{g}_{0} g_{n} \varphi=\varphi \tilde{g}_{0}$.

4e SÉRIE - TOME $39-2006-\mathrm{N}^{\circ} 2$ 
If $t \geqslant 2$ and if the element $\tilde{g}_{t-1}$ exists, then we can replace the triple $\left(n, t, g_{n}\right)$ by the triple $\left(n+t-1,1, \tilde{g}_{t-1} g_{n} \varphi \tilde{g}_{t-1}^{-1} \varphi^{-1}\right)$. Thus using induction on $t \in \mathbf{N}$, to prove the first part of the claim we can assume that $t=1$. As $W(k)$ is $p$-adically complete, the second part of the claim follows from its first part; this is so as we can take $\tilde{g}_{0}$ to be an infinite product of the form $\ldots \tilde{h}_{3} \tilde{h}_{2} \tilde{h}_{1}$ that has the property that for all $c \in \mathbf{N}$ the element $\tilde{h}_{c} \in G^{\prime}(W(k))=G(W(k))$ is congruent $\bmod p^{n-n(G)+c-1-m}$ to $1_{M^{\prime}}$ and moreover $\tilde{h}_{c} \tilde{h}_{c-1} \cdots \tilde{h}_{1} g_{n} \varphi \tilde{h}_{1}^{-1} \ldots \tilde{h}_{c}^{-1} \varphi^{-1} \in$ $G^{\prime}(W(k))=G(W(k))$ is congruent $\bmod p^{n-n(G)+c}$ to $1_{M^{\prime}}$. Thus to prove the claim, it suffices to prove its first part for $t=1$.

For $t=1$ we will use what we call the stairs method for $E$. Let $z_{n} \in \operatorname{Lie}\left(G^{\prime}\right)$ be such that $g_{n}$ is congruent $\bmod p^{n-n(G)+1}$ to $1_{M^{\prime}}+p^{n-n(G)} z_{n}$. As $n-n(G) \geqslant m+1$, based on 3.1(a) we can write

$$
p^{n-n(G)} z_{n}=\sum_{l \in S(1, v)} p^{u_{l}} c_{l} e_{l},
$$

where $u_{l} \in \mathbf{N}$ depends only on the cycle of $\pi$ to which $l$ belongs and where $c_{l} \in W(k)$. We take the $u_{l}$ 's to be the maximal possible values subject to the last sentence. Thus

$$
u_{l} \geqslant n-n(G)-m \geqslant m+\varepsilon_{p} \geqslant \varepsilon_{p} \geqslant 1 .
$$

From (13a) and (12) we get

$$
\min \left\{u_{l}+u_{l^{\prime}} \mid l, l^{\prime} \in S(1, v)\right\} \geqslant 2(n-n(G)-m) \geqslant n-n(G)+\varepsilon_{p} \geqslant n-n(G)+1 .
$$

Due to (13b), the product $\tilde{g}_{n}^{(1)}:=\prod_{l \in S(1, v)}\left(1_{M^{\prime}}+p^{u_{l}} c_{l} e_{l}\right) \in \mathbf{G L}_{M^{\prime}}(W(k))$ is congruent $\bmod p^{2(n-n(G)-m)}$ and so also $\bmod p^{n-n(G)+1}$ to $1_{M^{\prime}}+\sum_{l \in S(1, v)} p^{u_{l}} c_{l} e_{l}=1_{M^{\prime}}+$ $p^{n-n(G)} z_{n}$. The element $\tilde{g}_{n}^{(2)}:=\left(\tilde{g}_{n}^{(1)}\right)^{-1} g_{n} \in \mathbf{G L}_{M^{\prime}}(W(k))$ is congruent $\bmod p^{n-n(G)+1}$ to $\left(1_{M^{\prime}}+p^{n-n(G)} z_{n}\right)^{-1}\left(1_{M^{\prime}}+p^{n-n(G)} z_{n}\right)=1_{M^{\prime}}$. We have $g_{n}=\tilde{g}_{n}^{(1)} \tilde{g}_{n}^{(2)}$. For $l \in S(1, v)$ let $q_{l}:=-\min \left\{0, n_{l}\right\} \in \mathbf{N} \cup\{0\}$. We will choose $\tilde{g}_{1} \in G^{\prime}(W(k))$ to be a product $\prod_{l \in S(1, v)} \exp \left(p^{u_{l}+q_{l}} x_{l} e_{l}\right)$, with all $x_{l}$ 's in $W(k)$. This last product makes sense, cf. 2.6(a) and (b) and the fact that for $p=2$ we have $u_{l}+q_{l} \geqslant u_{l} \geqslant m+\varepsilon_{2} \geqslant \varepsilon_{2}=2$.

For $l \in S(1, v)$ we have $u_{l}=u_{\pi(l)}$. Thus

$$
\begin{aligned}
\varphi \tilde{g}_{1}^{-1} \varphi^{-1} & =\prod_{l \in S(1, v)} \exp \left(-p^{u_{l}+q_{l}} \sigma\left(x_{l}\right) \varphi\left(e_{l}\right)\right) \\
& =\prod_{l \in S(1, v)} \exp \left(-p^{u_{l}+q_{\pi^{-1}(l)}+n_{\pi^{-1}(l)}} \sigma\left(x_{\pi^{-1}(l)}\right) e_{l}\right) .
\end{aligned}
$$

These exponential elements are well defined even if $p=2$, as for $p=2$ we have inequalities $u_{l}+q_{\pi^{-1}(l)}+n_{\pi^{-1}(l)} \geqslant u_{l} \geqslant \varepsilon_{2} \geqslant 2$. Thus $\varphi \tilde{g}_{1}^{-1} \varphi^{-1} \in G^{\prime}(W(k))=G(W(k))$, cf. 2.6(c).

We have $\varphi \tilde{g}_{1}^{-1} \varphi^{-1} \in G^{\prime}(W(k))=G(W(k))$, cf. 2.6(c). We have to show that we can choose the $x_{l}$ 's such that $\tilde{g}_{1} g_{n} \varphi \tilde{g}_{1}^{-1} \varphi^{-1} \in G^{\prime}(W(k))$ is congruent $\bmod p^{n-n(G)+1}$ to $1_{M^{\prime}}$. It suffices to show that if $g_{n}$ is not congruent $\bmod p^{n-n(G)+1}$ to $1_{M^{\prime}}$, then we can choose the $x_{l}$ 's such that by replacing $g_{n}$ with $g_{n+1} \tilde{g}_{1} g_{n} \varphi \tilde{g}_{1}^{-1} \varphi^{-1} \in G^{\prime}(W(k))$, where $g_{n+1} \in G^{\prime}(W(k))$ is congruent $\bmod p^{n-n(G)+1}$ to $1_{M^{\prime}}$, we can also replace each $u_{l}$ by $u_{l}+t_{l}$, where $t_{l} \in \mathbf{N}$ depends only on the cycle of $\pi$ to which $l$ belongs.

The element $\tilde{g}_{1} g_{n} \varphi \tilde{g}_{1}^{-1} \varphi^{-1} \in G^{\prime}(W(k))$ is congruent $\bmod p^{n-n(G)+1}$ to the product $\tilde{g}_{1} \tilde{g}_{n}^{(1)} \varphi \tilde{g}_{1}^{-1} \varphi^{-1}$. From (13a), (13b), (14), and 2.6(a) and (b), we get that $\varphi \tilde{g}_{1}^{-1} \varphi^{-1}$ is congruent

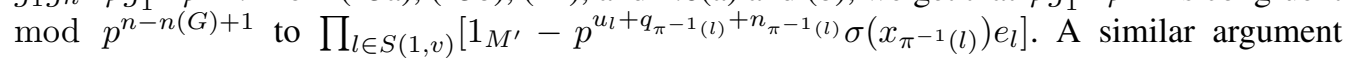


shows that $\tilde{g}_{1}$ is congruent $\bmod p^{n-n(G)+1}$ to $\prod_{l \in S(1, v)}\left(1_{M^{\prime}}+p^{u_{l}+q_{l}} x_{l} e_{l}\right)$. Thus the product $\tilde{g}_{1} \tilde{g}_{n}^{(1)} \varphi \tilde{g}_{1}^{-1} \varphi^{-1}$ of the three elements $\tilde{g}_{1}, \tilde{g}_{n}^{(1)}$, and $\varphi \tilde{g}_{1}^{-1} \varphi^{-1}$, is congruent $\bmod p^{n-n(G)+1}$ to the following product of three elements

$$
\begin{aligned}
& \prod_{l \in S(1, v)}\left(1_{M^{\prime}}+p^{u_{l}+q_{l}} x_{l} e_{l}\right) \prod_{l \in S(1, v)}\left(1_{M^{\prime}}+p^{u_{l}} c_{l} e_{l}\right) \\
& \quad \times \prod_{l \in S(1, v)}\left[1_{M^{\prime}}-p^{u_{l}+q_{\pi^{-1}(l)}+n_{\pi^{-1}(l)}} \sigma\left(x_{\pi^{-1}(l)}\right) e_{l}\right]
\end{aligned}
$$

and so (cf. (13b)) also to $1_{M^{\prime}}+\sum_{l \in S(1, v)} p^{u_{l}}\left[p^{q_{l}} x_{l}+c_{l}-p^{q_{\pi-1}(l)}+n_{\pi^{-1}(l)} \sigma\left(x_{\pi^{-1}(l)}\right)\right] e_{l}$.

To show that we can take each $t_{l}$ to be at least 1 , it suffices to show that we can choose the $x_{l}$ 's such that we have

$$
p^{q_{l}} x_{l}+c_{l}-p^{q_{\pi^{-1}(l)}+n_{\pi^{-1}(l)}} \sigma\left(x_{\pi^{-1}(l)}\right) \in p W(k) \quad \forall l \in S(1, v) .
$$

In other words, by denoting with $\bar{x} \in k$ the reduction mod $p$ of an arbitrary element $x \in W(k)$, it suffices to show that for each cycle $\pi_{0}=\left(l_{1}, \ldots, l_{q}\right)$ of $\pi$ there exist solutions in $k$ of the following circular system of equations over $k$

$$
\bar{b}_{l_{j}} \bar{x}_{l_{j}}+\bar{c}_{l_{j}}-\bar{d}_{l_{j}} \bar{x}_{l_{j-1}}^{p}=0 \quad \text { with } j \in S(1, q),
$$

where $b_{l_{j}}:=p^{q_{l_{j}}}$ and $d_{l_{j}}:=p^{q_{l_{j-1}}+n_{l_{j-1}}}$ (here we have $l_{0}=l_{q}$, cf. end of 2.1).

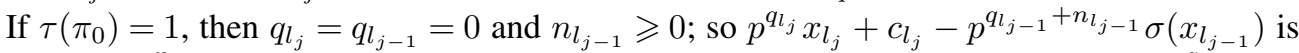
$x_{l_{j}}+c_{l_{j}}-p^{n_{l_{j-1}}} \sigma\left(x_{l_{j-1}}\right)$. If $\tau\left(\pi_{0}\right)=-1$, then $q_{l_{j-1}}=-n_{l_{j-1}} \geqslant 0$ and so we have $p^{q_{l_{j}}} x_{l_{j}}+$ $c_{l_{j}}-p^{q_{l_{j-1}}+n_{l_{j-1}}} \sigma\left(x_{l_{j-1}}\right)=p^{q_{l_{j}}} x_{l_{j}}+c_{l_{j}}-\sigma\left(x_{l_{j-1}}\right)$; moreover, there exists $j_{0} \in S(1, j)$ such that $q_{l_{j_{0}}}=-n_{l_{j_{0}}}>0$. Thus depending on the value of $\tau\left(\pi_{0}\right)$ we have:

(+) $\bar{b}_{l_{j}}=1$ and $\bar{d}_{l_{j}} \in\{0,1\}$ for all $j \in S(1, q)$, if $\tau\left(\pi_{0}\right)=1$;

(-) $\bar{d}_{l_{j}}=1$ and $\bar{b}_{l_{j}} \in\{0,1\}$ for all $j \in S(1, q)$ and moreover there exists $j_{0} \in S(1, q)$ such that $\bar{b}_{l_{j_{0}}}=0$, if $\tau\left(\pi_{0}\right)=-1$.

If $\tau\left(\pi_{0}\right)=1$, then based on (+) we can eliminate the variables $\bar{x}_{l_{q}}, \bar{x}_{l_{q-1}}, \ldots, \bar{x}_{l_{3}}$, and $\bar{x}_{l_{2}}$ one by one from the system (16). The resulting equation in the variable $\bar{x}_{l_{1}}$ is of the form $\bar{x}_{l_{1}}=\bar{u}_{l_{1}}+\bar{v}_{l_{1}} \bar{x}_{l_{1}}^{p^{q}}$, where $\bar{u}_{l_{1}}$ and $\bar{v}_{l_{1}} \in k$. This equation defines an étale $k$-algebra. Thus (as $k$ is separably closed) the system (16) has solutions in $k$ if $\tau\left(\pi_{0}\right)=1$.

If $\tau\left(\pi_{0}\right)=-1$, then based on (-) (and on the fact that $k$ is perfect) the values of $\bar{x}_{l_{j_{0}-1}}$, $\bar{x}_{l_{j_{0}-2}}, \ldots, \bar{x}_{l_{1}}, \bar{x}_{l_{q}}, \bar{x}_{l_{q-1}}, \ldots, \bar{x}_{l_{j_{0}}}$ are one by one uniquely determined and so the system (16) has a unique solution.

This ends the proof of the first part of the claim for $t=1$ and so it also ends the proof of the claim.

\subsubsection{Inequalities involving $s$ - and $h$-numbers}

Let $s_{L}^{\prime}$ and $h_{L}^{\prime}$ be the $s$-number and the $h$-number (respectively) of $\left(\operatorname{Lie}\left(G^{\prime}\right), \varphi\right)$. Let $s_{L}$ and $h_{L}$ be the $s$-number and the $h$-number (respectively) of $\left(\operatorname{Lie}\left(G_{B(k)}\right) \cap \operatorname{End}(M), \varphi\right)$. We recall from 2.7 that $d_{\mathrm{sm}} \in \mathbf{N} \cup\{0\}$ is the smallest number such that we have inclusions

$$
p^{d_{\mathrm{sm}}}\left(\operatorname{Lie}\left(G_{B(k)}\right) \cap \operatorname{End}(M)\right) \subseteq \operatorname{Lie}\left(G^{\prime}\right) \subseteq \operatorname{Lie}\left(G_{B(k)}\right) \cap \operatorname{End}(M) .
$$

We have $p^{s_{L}} \varphi\left(\operatorname{Lie}\left(G_{B(k)}\right) \cap \operatorname{End}(M)\right) \subseteq \operatorname{Lie}\left(G_{B(k)}\right) \cap \operatorname{End}(M)$. Thus $p^{s_{L}+d_{\text {sm }}} \varphi\left(\operatorname{Lie}\left(G^{\prime}\right)\right) \subseteq$ $p^{s_{L}+d_{\mathrm{sm}}} \varphi\left(\operatorname{Lie}\left(G_{B(k)}\right) \cap \operatorname{End}(M)\right) \subseteq p^{d_{\mathrm{sm}}}\left(\operatorname{Lie}\left(G_{B(k)}\right) \cap \operatorname{End}(M)\right) \subseteq \operatorname{Lie}\left(G^{\prime}\right)$. From the very 
definition of $s_{L}^{\prime}$ we get

$$
s_{L}^{\prime} \leqslant s_{L}+d_{\mathrm{sm}}
$$

The $h$-numbers of $\left(\operatorname{Lie}\left(G_{B(k)}\right) \cap \operatorname{End}(M), p^{\max \left\{s_{L}, s_{L}^{\prime}\right\}} \varphi\right)$ and $\left(\operatorname{Lie}\left(G^{\prime}\right), p^{\max \left\{s_{L}, s_{L}^{\prime}\right\}} \varphi\right)$ are $h_{L}+\max \left\{s_{L}, s_{L}^{\prime}\right\}-s_{L}$ and $h_{L}^{\prime}+\max \left\{s_{L}, s_{L}^{\prime}\right\}-s_{L}^{\prime}$ (respectively). From this and (17a) we easily get that

$$
h_{L}^{\prime}+\max \left\{s_{L}, s_{L}^{\prime}\right\}-s_{L}^{\prime} \leqslant d_{\mathrm{sm}}+h_{L}+\max \left\{s_{L}, s_{L}^{\prime}\right\}-s_{L} .
$$

From (17b), (17c), and the inequalities $d_{\mathrm{sm}} \leqslant n(G) \leqslant \delta(G)$ (see 2.7.2(c) and 2.7), we get

$$
h_{L}^{\prime} \leqslant h_{L}+d_{\mathrm{sm}}+s_{L}^{\prime}-s_{L} \leqslant h_{L}+d_{\mathrm{sm}}+d_{\mathrm{sm}} \leqslant h_{L}+2 \delta(G) .
$$

\subsubsection{End of the proof of 1.2}

As $v=\operatorname{dim}\left(G_{B(k)}\right), G^{\prime}$, and $n(G)$ depend only on $G$ and as $s_{L}^{\prime}$ and $h_{L}^{\prime}$ depend only on the family $\left\{(M, g \varphi, G) \mid g \in G(W(k))=G^{\prime}(W(k))\right\}$ of latticed $F$-isocrystals with a group over $k$, the number

$$
n_{\text {fam }}:=2 d\left(v, s_{L}^{\prime}, h_{L}^{\prime}\right)+\varepsilon_{p}+n(G)
$$

is not changed if $\varphi$ gets replaced by $g \varphi$ for some $g \in G(W(k))$. As $m \leqslant d\left(v, s_{L}^{\prime}, h_{L}^{\prime}\right)$ (cf. 2.4.1), we have $n_{\text {fam }} \geqslant 2 m+\varepsilon_{p}+n(G)$. So from (12) applied with $n=n_{\text {fam }}$ and from 3.1.1, we get that for any $g_{n_{\mathrm{fam}}} \in G(W(k))$ congruent $\bmod p^{n_{\mathrm{fam}}}$ to $1_{M}$, there exists an isomorphism between $(M, \varphi, G)$ and $\left(M, g_{n_{\mathrm{fam}}} \varphi, G\right)$ defined by an element of $G^{\prime}(W(k))=G(W(k))$. This property holds even if $\varphi$ gets replaced by $g \varphi$. As $n(G) \leqslant \delta(G)$, we have

$$
n_{\text {fam }} \leqslant 2 d\left(v, s_{L}^{\prime}, h_{L}^{\prime}\right)+\varepsilon_{p}+\delta(G) .
$$

So based on the effectiveness part of 2.4.1 and on (17b) and (17d), to end the proof of 1.2 it is enough to show that $\delta(G), s_{L}$, and $h_{L}$ are effectively bounded from above. But $\delta(G)$ is effectively computable in terms of the ideal sheaf of $\mathcal{O}_{\mathbf{G L}_{M}}$ that defines the closed embedding homomorphism $G \hookrightarrow \mathbf{G L}_{M}$, cf. [2, 3.3, Lemma 2]. As the numbers $s_{L}$ and $h_{L}$ are effectively computable in terms of $\left(\operatorname{Lie}\left(G_{B(k)}\right), \varphi\right)$ and $\operatorname{End}(M)$ and as the connected group $G_{B(k)}$ is uniquely determined by its Lie algebra (cf. [1, Ch. II, 7.1]), the numbers $s_{L}$ and $h_{L}$ are also effectively computable in terms of the closed embedding homomorphism $G \hookrightarrow \mathbf{G L}_{M}$. This ends the proof of 1.2 .

3.1.4. Definition. - Let $(M, \varphi, G)$ be a latticed $F$-isocrystal with a group over $k$. By the isomorphism number (to be abbreviated as the $i$-number) of $(M, \varphi, G)$ we mean the smallest number $n \in \mathbf{N} \cup\{0\}$ such that for any $g_{n} \in G(W(\bar{k}))$ congruent $\bmod p^{n}$ to $1_{M \otimes_{W(k)} W(\bar{k})}$, there exists an isomorphism between $\left(M \otimes_{W(k)} W(\bar{k}), \varphi \otimes \sigma_{\bar{k}}, G_{W(\bar{k})}\right)$ and $\left(M \otimes_{W(k)} W(\bar{k}), g_{n}(\varphi \otimes\right.$ $\left.\left.\sigma_{\bar{k}}\right), G_{W(\bar{k})}\right)$ which is an element of $G(W(\bar{k}))$. If $G=\mathbf{G L}_{M}$, we also refer to $n$ as the $i$-number of $(M, \varphi)$.

If $\left(M, \varphi, G, \lambda_{M}\right)$ and $G^{0}$ are as in 2.2.1(c), then by the $i$-number of $\left(M, \varphi, G, \lambda_{M}\right)$ we mean the $i$-number of its latticed $F$-isocrystal with a group $\left(M, \varphi, G^{0}\right)$ over $k$.

3.1.5. Example. - Suppose $G$ is smooth over $\operatorname{Spec}(W(k))$ and $k=\bar{k}$. Thus $n(G)=d_{\mathrm{sm}}=0$, $s_{L}^{\prime}=s_{L}, h_{L}^{\prime}=h_{L}$, and $n_{\mathrm{fam}}=2 d\left(v, s_{L}, h_{L}\right)+\varepsilon_{p}$. Let $m:=\mathbf{T}\left(\operatorname{Lie}\left(G^{\prime}\right), \varphi\right)$. If $n$ is as in 3.1.4, then we have $0 \leqslant n \leqslant 2 m+\varepsilon_{p} \leqslant 2 d\left(v, s_{L}, h_{L}\right)+\varepsilon_{p}$ (cf. 3.1.1 and 3.1.3). 


\subsection{Truncations}

In 3.2.1 to 3.2.7 we work in a context that pertains to Dieudonné modules and to $p$-divisible groups. In particular, in 3.2.1 and 3.2.2 we define and study $D$-truncations that are the crystalline analogues (with a group) over $k$ of truncated Barsotti-Tate groups over $\operatorname{Spec}(k)$. In 3.2.8 we consider reductions modulo powers of $p$ of those $F$-crystals with a group $(M, \varphi, G)$ over $k$ for which $G$ is smooth over $\operatorname{Spec}(W(k))$. In 3.2.9 we introduce the $F$-truncations that generalize the $D$-truncations.

\subsubsection{On $D$-truncations}

Let $(r, d) \in \mathbf{N} \times(\mathbf{N} \cup\{0\})$. Let $(M, \varphi, G)$ be an $F$-crystal with a group over $k$. Until 3.2.8 we assume that $(M, \varphi)$ is a Dieudonné module over $k$, that $r_{M}=r$, that $d$ is the dimension of the kernel of $\varphi \bmod p$, that $G$ is smooth over $\operatorname{Spec}(W(k))$, and that the $W$-condition holds for $(M, \varphi, G)$. Let $M=\widetilde{F}^{0}(M) \oplus \widetilde{F}^{1}(M)$ be a direct sum decomposition such that $M=$ $\varphi\left(\widetilde{F}^{0}(M) \oplus \frac{1}{p} \widetilde{F}^{1}(M)\right)$ and the cocharacter $\mu: \mathbf{G}_{m} \rightarrow \mathbf{G L}_{M}$ that fixes $\widetilde{F}^{0}(M)$ and that acts via the inverse of the identical character of $\mathbf{G}_{m}$ on $\widetilde{F}^{1}(M)$, factors through $G$ (cf. 2.2.1(d)). The rank of $\widetilde{F}^{1}(M)$ is $d$. Let $\sigma_{0}:=\varphi \mu(p)$; it is a $\sigma$-linear automorphism of either $M$ and $\mathcal{T}(M)$ (cf. 2.5). As $\sigma_{0}$ normalizes $\operatorname{Lie}\left(G_{B(k)}\right)$ and $\operatorname{End}(M)$, it also normalizes $\operatorname{Lie}(G)=\operatorname{Lie}\left(G_{B(k)}\right) \cap \operatorname{End}(M)$. As $G(W(k))=G(B(k)) \cap \mathbf{G L}_{M}(W(k))$, we have $\sigma_{0} G(W(k)) \sigma_{0}^{-1}=G(W(k))$.

Let $q \in \mathbf{N}$. Let $\vartheta: M \rightarrow M$ be the $\sigma^{-1}$-linear endomorphism that is the Verschiebung map of $(M, \varphi)$; we have $\vartheta \varphi=\varphi \vartheta=p 1_{M}$. We denote also by $\vartheta$ its reduction mod $p^{q}$. By the $D$-truncation $\bmod p^{q}$ of $(M, \varphi, G)$ we mean the quadruple

$$
\left(M / p^{q} M, \varphi, \vartheta, G_{W_{q}(k)}\right) .
$$

This quadruple determines (respectively is determined by) the reduction $\bmod p^{q}$ (respectively $\left.\bmod p^{q+1}\right)$ of $(M, \varphi, G)$. We also refer to $\left(M / p^{q} M, \varphi, \vartheta\right)$ as the $D$-truncation $\bmod p^{q}$ of $(M, \varphi)$. If $\left(M_{1} / p^{q} M_{1}, \varphi_{1}, \vartheta_{1}, G_{1 W_{q}(k)}\right)$ is a similar $D$-truncation mod $p^{q}$, then by an isomorphism $f:\left(M / p^{q} M, \varphi, \vartheta, G_{W_{q}(k)}\right) \stackrel{\sim}{\longrightarrow}\left(M_{1} / p^{q} M_{1}, \varphi_{1}, \vartheta_{1}, G_{1 W_{q}(k)}\right)$ we mean a $W_{q}(k)$ linear isomorphism $f: M / p^{q} M \stackrel{\sim}{\sim} M_{1} / p^{q} M_{1}$ such that we have identities $f \varphi=\varphi_{1} f$ and $f \vartheta=\vartheta_{1} f$ and the isomorphism $\mathbf{G L}_{M / p^{q} M} \stackrel{\sim}{\longrightarrow} \mathbf{G L}_{M_{1} / p^{q} M_{1}}$ induced by $f$, takes $G_{W_{q}(k)}$ onto $G_{1 W_{q}(k)}$.

If $\left(M_{1}, \varphi_{1}, G_{1}\right)$ is $(M, g \varphi, G)$ with $g \in G(W(k))$ and if $f \in G\left(W_{q}(k)\right)$, then we say $f$ is an inner isomorphism between the $D$-truncation $\bmod p^{q}$ of $(M, \varphi, G)$ and $(M, g \varphi, G)$.

3.2.2. LEMMA. - For $g \in G(W(k))$ the following two statements are equivalent:

(a) the D-truncations $\bmod p^{q}$ of $(M, \varphi, G)$ and $(M, g \varphi, G)$ are inner isomorphic;

(b) there exists an element $\tilde{g} \in G(W(k))$ such that $\tilde{g} g \varphi \tilde{g}^{-1}=g_{q} \varphi$, where $g_{q} \in G(W(k))$ is congruent $\bmod p^{q}$ to $1_{M}$.

Proof. - As $G$ is smooth over $\operatorname{Spec}(W(k))$, the reduction homomorphism $G(W(k)) \rightarrow$ $G\left(W_{q}(k)\right)$ is onto. Thus it suffices to check that (a) implies (b) under the extra assumptions that the $\sigma$-linear endomorphisms $\varphi$ and $g \varphi$ coincide $\bmod p^{q}$ and that the $\sigma^{-1}$-linear endomorphisms $\vartheta$ and $\vartheta g^{-1}$ coincide mod $p^{q}$. Let $g_{0}:=\sigma_{0}^{-1} g \sigma_{0} \in G(W(k))$. Let $g_{0, q} \in G\left(W_{q}(k)\right)$ be $g_{0}$ $\bmod p^{q}$. We have $\varphi=\sigma_{0} \mu\left(p^{-1}\right)$ and $g \varphi=\sigma_{0} g_{0} \mu\left(p^{-1}\right)$. We get that the two endomorphisms $\mu\left(p^{-1}\right)$ and $g_{0} \mu\left(p^{-1}\right)$ of $M$ coincide $\bmod p^{q}$. Thus

(i) the element $g_{0, q}$ fixes $\widetilde{F}^{0}(M) / p^{q} \widetilde{F}^{0}(M)$ and is congruent $\bmod p^{q-1}$ to $1_{M / p^{q} M}$.

We have $\vartheta=p \mu(p) \sigma_{0}^{-1}$ and $\vartheta g^{-1}=p \mu(p) g_{0}^{-1} \sigma_{0}^{-1}$. We get that the two endomorphisms $p \mu(p)$ and $p \mu(p) g_{0}^{-1}$ of $M$ coincide mod $p^{q}$. Thus $p \mu(p)$ and $p \mu(p) g_{0}$ also coincide mod $p^{q}$ and therefore we have an inclusion

$4^{\text {e }}$ SÉRIE - TOME $39-2006-\mathrm{N}^{\circ} 2$ 
(ii) $\left(1_{M / p^{q} M}-g_{0, q}\right)\left(\widetilde{F}^{1}(M) / p^{q} \widetilde{F}^{1}(M)\right) \subseteq p^{q-1} \widetilde{F}^{0}(M) / p^{q} \widetilde{F}^{0}(M)$.

Let $\widetilde{F}^{-1}(\operatorname{End}(M))$ be the maximal direct summand of $\operatorname{End}(M)$ on which $\mu$ acts via the identity cocharacter of $\mathbf{G}_{m}$. Thus $\widetilde{F}^{-1}(\operatorname{End}(M))$ is the $\operatorname{Hom}\left(\widetilde{F}^{1}(M), \widetilde{F}^{0}(M)\right)$ factor of the following direct sum decomposition $\operatorname{End}(M)=\operatorname{End}\left(\widetilde{F}^{1}(M)\right) \oplus \operatorname{End}\left(\widetilde{F}^{0}(M)\right) \oplus$ $\operatorname{Hom}\left(\widetilde{F}^{1}(M), \widetilde{F}^{0}(M)\right) \oplus \operatorname{Hom}\left(\widetilde{F}^{0}(M), \widetilde{F}^{1}(M)\right)$ into $W(k)$-submodules.

Let $U_{-1}^{\text {big }}$ be the closed subgroup scheme of $\mathbf{G L}_{M}$ defined by the rule: if $A$ is a commutative $W(k)$-algebra, then $U_{-1}^{\text {big }}(A):=1_{M \otimes_{W(k)} A}+\widetilde{F}^{-1}(\operatorname{End}(M)) \otimes_{W(k)} A \leqslant \mathbf{G L}_{M}(A)$. So $U_{-1}^{\text {big }}$ is the maximal subgroup scheme of $\mathbf{G L}_{M}$ that fixes both $\widetilde{F}^{0}(M)$ and $M / \widetilde{F}^{0}(M)$; it is smooth over $\operatorname{Spec}(W(k))$. We have $\operatorname{Lie}\left(U_{-1}^{\text {big }}\right)=\widetilde{F}^{-1}(\operatorname{End}(M))$. Let $U_{-1}$ be the closed subgroup scheme of $U_{-1}^{\text {big }}$ defined by the rule:

$$
U_{-1}(A):=1_{M \otimes_{W(k)} A}+\left(\operatorname{Lie}(G) \cap \widetilde{F}^{-1}(\operatorname{End}(M))\right) \otimes_{W(k)} A .
$$

The group scheme $U_{-1}$ is smooth, unipotent, has connected fibres and its Lie algebra is the direct summand $\operatorname{Lie}(G) \cap \widetilde{F}^{-1}(\operatorname{End}(M))$ of $\widetilde{F}^{-1}(\operatorname{End}(M))$. As $U_{-1 B(k)}$ is connected and $\operatorname{Lie}\left(U_{-1}\right) \subseteq \operatorname{Lie}(G)$, the group $U_{-1 B(k)}$ is a closed subgroup of $G_{B(k)}$ (cf. [1, Ch. II, 7.1]). Thus $U_{-1}$ is a closed subgroup scheme of $G$. As $\mu$ factors through $G$, we have two identities $\operatorname{Lie}(G) \cap \operatorname{Lie}\left(U_{-1}^{\text {big }}\right)=\operatorname{Lie}\left(U_{-1}\right)$ and $\operatorname{Lie}\left(G_{k}\right) \cap \operatorname{Lie}\left(U_{-1 k}^{\text {big }}\right)=\operatorname{Lie}\left(U_{-1 k}\right)$. Thus the intersection $U_{-1}^{\prime}:=G \cap U_{-1}^{\text {big }}$ has smooth fibres and has $U_{-1}$ as its identity component. As the group $U_{-1 B(k)}^{\text {big }} / U_{-1 B(k)}$ has no non-trivial finite subgroups, we have $U_{-1 B(k)}=U_{-1 B(k)}^{\prime}$. As $U_{-1}^{\text {big }}$ is a complete intersection in $\mathbf{G L}_{M}, U_{-1}^{\prime}$ has dimension at least equal to $1+\operatorname{dim}\left(U_{-1 k}\right)$ at each $k$-valued point. So as $\operatorname{dim}\left(U_{-1 k}^{\prime}\right)=\operatorname{dim}\left(U_{k}\right)$, the group $U_{-1 B(k)}^{\prime}=U_{-1 B(k)}$ is Zariski dense in $U_{-1}^{\prime}$. Thus $U_{-1}=U_{-1}^{\prime}=G \cap U_{-1}^{\text {big }}$.

We have $g_{0, q} \in \operatorname{Ker}\left(U_{-1}^{\text {big }}\left(W_{q}(k)\right) \rightarrow U_{-1}^{\text {big }}\left(W_{q-1}(k)\right)\right)$, cf. (i) and (ii). As $U_{-1}=G \cap U_{-1}^{\text {big }}$, in fact we have $g_{0, q} \in \operatorname{Ker}\left(U_{-1}\left(W_{q}(k)\right) \rightarrow U_{-1}\left(W_{q-1}(k)\right)\right)$. Thus, up to a replacement of $g$ by a $\operatorname{Ker}\left(G(W(k)) \rightarrow G\left(W_{q}(k)\right)\right)$-multiple of it, we can assume that $g_{0} \in U_{-1}(W(k))$. We write $g_{0}=1_{M}+p^{q-1} u_{-1}$, where $u_{-1} \in \operatorname{Lie}\left(U_{-1}\right)$.

Let $\tilde{g}:=1_{M}+p^{q} u_{-1} \in U_{-1}(W(k)) \leqslant G(W(k))$. As we have $\tilde{g}^{-1}=1_{M}-p^{q} u_{-1}$ and $g_{0}^{-1}=1_{M}-p^{q-1} u_{-1}$, we get $\mu\left(p^{-1}\right) \tilde{g}^{-1} \mu(p)=g_{0}^{-1}$. Thus we compute that $\tilde{g} g \varphi \tilde{g}^{-1}$ is $\tilde{g} g \sigma_{0} \mu\left(p^{-1}\right) \tilde{g}^{-1} \mu(p) \sigma_{0}^{-1} \varphi=\tilde{g} g \sigma_{0} g_{0}^{-1} \sigma_{0}^{-1} \varphi=\tilde{g} g\left(\sigma_{0} g \sigma_{0}^{-1}\right)^{-1} \varphi=\tilde{g} g g^{-1} \varphi=\tilde{g} \varphi$. So as $g_{q}$ we can take $\tilde{g}$. Thus (a) implies (b). Obviously (b) implies (a).

3.2.3. Corollary. - Suppose $k=\bar{k}$ and $(M, \varphi)$ is the Dieudonné module of a p-divisible group $D$ over $\operatorname{Spec}(k)$ of height $r=r_{M}$ and dimension d. Let $n \in \mathbf{N} \cup\{0\}$ be the $i$-number of $(M, \varphi)$. Then $n$ is the smallest number $t \in \mathbf{N} \cup\{0, \infty\}$ for which the following statement holds:

$(*)$ if $D_{1}$ is a p-divisible group over $\operatorname{Spec}(k)$ of height $r$ and dimension $d$ and if $D_{1}\left[p^{t}\right]$ is isomorphic to $D\left[p^{t}\right]$, then $D_{1}$ is isomorphic to $D$.

Proof. - The Dieudonné module of $D_{1}$ is isomorphic to $(M, g \varphi)$ for some $g \in \mathbf{G L}_{M}(W(k))$; moreover any such pair $(M, g \varphi)$ is isomorphic to the Dieudonné module of some $p$-divisible group over $\operatorname{Spec}(k)$ of height $r$ and dimension $d$. For $q \in \mathbf{N}$, the classical Dieudonné theory achieves also a natural one-to-one and onto correspondence between the isomorphism classes of truncated Barsotti-Tate groups of level $q$ over $k$ and the isomorphism classes of $D$-truncations $\bmod p^{q}$ of Dieudonné modules over $k$ (see [14, pp. 153 and 160]). So $(*)$ holds if and only if for any $g \in \mathbf{G L}_{M}(W(k))$, the fact that the $D$-truncations $\bmod p^{t}$ of $(M, \varphi)$ and $(M, g \varphi)$ are isomorphic implies that $(M, \varphi)$ and $(M, g \varphi)$ are isomorphic. From this and 3.2.2 (applied with $G=\mathbf{G L}_{M}$ and $\left.q=t\right)$, we get that $(*)$ holds if and only if $(M, \varphi)$ and $(M, g \varphi)$ are isomorphic for all elements $g \in \mathbf{G L}_{M}(W(k))$ congruent $\bmod p^{t}$ to $1_{M}$. Thus $(*)$ holds if and only if $t \geqslant n$. 


\subsubsection{Proof of 1.3}

To prove 1.3, we can assume that $k=\bar{k}$ and that $(M, \varphi)$ is the Dieudonné module of $D$. Let $n$ be the $i$-number of $(M, \varphi)$. Let $n_{\text {fam }}$ be as in 3.1.5 for $G=\mathbf{G L}_{M}$. We have $n \leqslant n_{\mathrm{fam}}$, cf. 3.1.5. From 3.2 .3 we get that $D$ is uniquely determined up to isomorphism by $D\left[p^{n}\right]$ and so also by $D\left[p^{n_{\mathrm{fam}}}\right]$. Thus the number $T(r, d)$ exists and we have $T(r, d) \leqslant n_{\mathrm{fam}}$. From 3.2 .3 we also get that $T(r, d) \geqslant n$. If $d \in\{0, r\}$, then $T(r, d)=n_{\text {fam }}=n=0$. If $d \notin\{0, r\}$, then the $s$-number and the $h$-number of $(\operatorname{End}(M), \varphi)$ are 1 and 2 (respectively) and $\operatorname{End}(M)$ has rank $r^{2}$. Thus for $d \in S(1, r-1)$ we have (cf. 3.1.5)

$$
n \leqslant T(r, d) \leqslant n_{\text {fam }} \leqslant 2 d\left(r^{2}, 1,2\right)+\varepsilon_{p} .
$$

So $T(r, d)$ is effectively bounded from above in terms of $r$, cf. 2.4.1. This proves 1.3.

\subsubsection{Principal quasi-polarizations}

Suppose $r=2 d=r_{M}, k=\bar{k}$, and we have a principal quasi-polarization $\lambda_{M}$ of $(M, \varphi)$. We refer to the triple $\left(M, \varphi, \lambda_{M}\right)$ as a principally quasi-polarized Dieudonné module over $k$. Let $G:=\mathbf{S p}\left(M, \lambda_{M}\right)$. Let $n \in \mathbf{N} \cup\{0\}$ be the $i$-number of $(M, \varphi, G)$. Let $\left(D, \lambda_{D}\right)$ be a principally quasi-polarized $p$-divisible group over $\operatorname{Spec}(k)$ whose principally quasi-polarized Dieudonné module is isomorphic to $\left(M, \varphi, \lambda_{M}\right)$. The principally quasi-polarized Dieudonné module of any other principally quasi-polarized $p$-divisible group over $\operatorname{Spec}(k)$ of height $r=r_{M}$ is of the form $\left(M, g \varphi, \lambda_{M}\right)$ for some $g \in G(W(k))$. So as in the proof of 3.2.3 we argue that $n$ is the smallest number $t \in \mathbf{N} \cup\{0, \infty\}$ for which the following statement holds:

$(*)$ if $\left(D_{1}, \lambda_{D_{1}}\right)$ is a principally quasi-polarized p-divisible group over $\operatorname{Spec}(k)$ of height $r=2 d$ and if the principally quasi-polarized truncated Barsotti-Tate groups of level t of $\left(D_{1}, \lambda_{D_{1}}\right)$ and $\left(D, \lambda_{D}\right)$ are isomorphic, then $\left(D_{1}, \lambda_{D_{1}}\right)$ is isomorphic to $\left(D, \lambda_{D}\right)$.

As in 3.2.4 we argue that there exists a smallest number $T(d) \in \mathbf{N}$ such that any principally quasi-polarized $p$-divisible group over $\operatorname{Spec}(k)$ of height $r=2 d$ is uniquely determined up to isomorphism by its principally quasi-polarized truncated Barsotti-Tate group of level $T(d)$. The number $T(d)$ is effectively bounded from above in terms of the relative dimension $2 d^{2}+d$ of $G=\mathbf{S p}\left(M, \lambda_{M}\right)$ over $\operatorname{Spec}(W(k))$ and so also of $d$ itself.

From the very definition of $T(d)$ we get:

3.2.6. COROLlaRY.-Suppose $k=\bar{k}$. Let $D$ be a p-divisible group over $\operatorname{Spec}(k)$ of height $r=2 d$ and dimension $d$. Let $D^{\mathrm{t}}$ be the Cartier dual of $D$. Then the number of isomorphism classes of principally quasi-polarized p-divisible groups of the form $\left(D, \lambda_{D}\right)$ is bounded from above by the finite number of distinct truncations of level $T(d)$ of isomorphisms $D \stackrel{\sim}{\longrightarrow} D^{\mathrm{t}}$, i.e. by the number of elements of the following finite set of isomorphisms $\operatorname{Im}\left(\operatorname{Isom}\left(D, D^{\mathrm{t}}\right) \rightarrow \operatorname{Isom}\left(D\left[p^{T(d)}\right], D^{\mathrm{t}}\left[p^{T(d)}\right]\right)\right)$.

3.2.7. Proposition. - Suppose $k=\bar{k}$. Let $R$ be the normalization of $k[[w]]$ in an algebraic closure $K$ of $k((w))$. For $* \in\{k, K\}$ let $D_{*}$ be a p-divisible group over $\operatorname{Spec}(*)$ of height $r$ and dimension $d$. Then $D_{k}$ is the specialization (via $\operatorname{Spec}(R)$ ) of a p-divisible group over $\operatorname{Spec}(K)$ which is isomorphic to $D_{K}$ if and only if $D_{k}\left[p^{T(r, d)}\right]$ is the specialization (via $\operatorname{Spec}(R)$ ) of a truncated Barsotti-Tate group of level $T(r, d)$ over $\operatorname{Spec}(K)$ which is isomorphic to $D_{K}\left[p^{T(r, d)}\right]$.

Proof. - It suffices to check the if part. Let $\mathcal{G}_{R}$ be a truncated Barsotti-Tate group of level $T(r, d)$ over $\operatorname{Spec}(R)$ that lifts $D_{k}\left[p^{T(r, d)}\right]$ and that has the property that its fibre over $\operatorname{Spec}(K)$ is isomorphic to $D_{K}\left[p^{T(r, d)}\right]$. Let $R_{0}$ be a finite $k[[w]]$-subalgebra of $R$ such that $\mathcal{G}_{R}$ is the pullback of a truncated Barsotti-Tate group $\mathcal{G}_{R_{0}}$ of level $T(r, d)$ over $\operatorname{Spec}\left(R_{0}\right)$. As $R_{0}$ is a complete discrete valuation ring, there exists a $p$-divisible group $D_{R_{0}}^{\prime}$ over $\operatorname{Spec}\left(R_{0}\right)$ that lifts 
both $D_{k}$ and $\mathcal{G}_{R_{0}}$ (cf. [21, 4.4(f)]). The pullback of $D_{R_{0}}^{\prime}$ to $\operatorname{Spec}(K)$ is isomorphic to $D_{K}$ (cf. $1.3)$ and it specializes $(\operatorname{via} \operatorname{Spec}(R))$ to $D_{k}$.

We have a logical variant of 3.2.7 in the principally quasi-polarized context. We next consider $F$-crystals with a group over $k$.

3.2.8. THEOREM. - Suppose $k=\bar{k}$. Let $(M, \varphi, G)$ be an F-crystal with a group over $k$; so $\varphi(M) \subseteq M$. Let $h$ be the $h$-number of $(M, \varphi)$. Let $m:=\mathbf{T}(\operatorname{Lie}(G), \varphi)$ and let $n:=2 m+\varepsilon_{p}$. Let $g \in G(W(k))$. Let $t \in \mathbf{N} \cup\{0\}$.

(a) Suppose $G$ is smooth over $\operatorname{Spec}(W(k))$ and there exists $\tilde{g}_{h+n+t} \in G\left(W_{h+n+t}(k)\right)$ which is an isomorphism between the reductions $\bmod p^{h+n+t}$ of $(M, g \varphi)$ and $(M, \varphi)$. Then there exists $\tilde{g}_{0} \in G(W(k))$ which is an isomorphism between $(M, g \varphi, G)$ and $(M, \varphi, G)$ and whose image in $G\left(W_{h+n+t}(k)\right)$ is congruent $\bmod p^{n-m+t}$ to $\tilde{g}_{h+n+t}$.

(b) If $G=\mathbf{G L}_{M}$, then the images of the two reduction homomorphisms $\operatorname{Aut}(M, \varphi) \rightarrow$ $\operatorname{Aut}\left(M / p^{n-m+t} M, \varphi\right)$ and $\operatorname{Aut}\left(M / p^{n+h+t} M, \varphi\right) \rightarrow \operatorname{Aut}\left(M / p^{n-m+t} M, \varphi\right)$ are the same.

Proof. - Part (b) is a practical application of (a) for the case when $G=\mathbf{G L}_{M}$. As $G(W(k))$ surjects onto $G\left(W_{h+n+t}(k)\right)$, it suffices to prove (a) under the extra assumption that $\tilde{g}_{h+n+t}=$ $1_{M / p^{h+n+t} M}$. So $g \bmod p^{h+n+t}$ fixes $\operatorname{Im}\left(\varphi(M) \rightarrow M / p^{h+n+t} M\right)$. But $p^{h} M \subseteq \varphi(M)$ and so $g \bmod p^{h+n+t}$ fixes also the $W_{h+n+t}(k)$-submodule $p^{h} M / p^{h+n+t} M$ of $M / p^{h+n+t} M$. Thus $g$ is congruent $\bmod p^{n+t}$ to $1_{M}$. So the element $\tilde{g}_{0}$ exists, cf. 3.1 .1 (applied with $G=G^{\prime}$, $M=M^{\prime}$, and $n(G)=0$ ).

\subsubsection{On $F$-truncations}

One can generalize the $D$-truncations of 3.2 .1 as follows. Let $(M, \varphi, G)$ be a $p$-divisible object with a group over $k$ and let $\left(F^{i}(M)\right)_{i \in S(a, b)}$ be a lift of it (see 2.2.1(b) and (d)). Let $\varphi_{i}: F^{i}(M) \rightarrow M$ be the $\sigma$-linear map defined by the rule $\varphi_{i}(x)=p^{-i} \varphi(x)$, where $x \in F^{i}(M)$. We denote also by $\varphi_{i}$ its reduction $\bmod p^{q}$. By an inner isomorphism between $\left(M / p^{q} M,\left(F^{i}(M) / p^{q} F^{i}(M)\right)_{i \in S(a, b)},\left(\varphi_{i}\right)_{i \in S(a, b)}, G_{W_{q}(k)}\right)$ and a similar quadruple $\left(M / p^{q} M,\left(F_{1}^{i}(M) / p^{q} F_{1}^{i}(M)\right)_{i \in S(a, b)},\left((g \varphi)_{i}\right)_{i \in S(a, b)}, G_{W_{q}(k)}\right)$ defined by some lift $\left(F_{1}^{i}(M)\right)_{i \in S(a, b)}$ of $(M, g \varphi, G)$ (with $g \in G(W(k))$ ), we mean an arbitrary element $f \in \operatorname{Im}\left(G(W(k)) \rightarrow G\left(W_{q}(k)\right)\right)$ that has the following two properties:

(i) it takes $F^{i}(M) / p^{q} F^{i}(M)$ onto $F_{1}^{i}(M) / p^{q} F_{1}^{i}(M)$ for all $i \in S(a, b)$;

(ii) we have $f \varphi_{i}=(g \varphi)_{i} f$ for all $i \in S(a, b)$.

By the $F$-truncation mod $p^{q}$ of $(M, \varphi, G)$ we mean the set $F_{q}(M, \varphi, G)$ of inner isomorphism classes of quadruples $\left(M / p^{q} M,\left(F^{i}(M) / p^{q} F^{i}(M)\right)_{i \in S(a, b)},\left(\varphi_{i}\right)_{i \in S(a, b)}, G_{W_{q}(k)}\right)$ we obtain by allowing $\left(F^{i}(M)\right)_{i \in S(a, b)}$ to run through all lifts of $(M, \varphi, G)$.

If $(M, \varphi)$ is a Dieudonné module over $k$ and if $G$ is smooth, then it is easy to see that the $D$-truncations $\bmod p^{q}$ of $(M, \varphi, G)$ and $(M, g \varphi, G)$ are inner isomorphic if and only if the $F$-truncations $\bmod p^{q}$ of $(M, \varphi, G)$ and $(M, g \varphi, G)$ are inner isomorphic.

\subsection{Refinements of 3.1.1}

In many particular cases we can choose the $W(k)$-span $E$ of 3.1.1(a) to be stable under products and this can lead to significant improvements of the inequalities we obtained in 3.1.1 to 3.1.5. For the sake of generality, we now formalize such improvements in a relative context.

Let $(M, \varphi, G)$ be a latticed $F$-isocrystal with a group over $k$. Until Section 4 we assume $k=\bar{k}$. Until Section 4 we also assume that there exists an integral, closed subgroup scheme $G_{1}$ of $\mathbf{G L}_{M}$ which contains $G$ and for which the following two conditions hold: 
(i) the triple $\left(M, \varphi, G_{1}\right)$ is a latticed $F$-isocrystal with a group over $k$;

(ii) there exist a $B(k)$-basis $\mathcal{B}_{1}:=\left\{e_{1}, \ldots, e_{v_{1}}\right\}$ of $\operatorname{Lie}\left(G_{1 B(k)}\right)$ and a permutation $\pi_{1}$ of $S\left(1, v_{1}\right)$, that have the following four properties:

(ii.a) $E_{1}:=\left\langle e_{1}, \ldots, e_{v_{1}}\right\rangle$ is a $W(k)$-submodule of $\operatorname{End}(M)$ such that $E_{1} E_{1} \subseteq E_{1}$;

(ii.b) each cycle $\left(l_{1}, \ldots, l_{q}\right)$ of $\pi_{1}$ has the property that for $j \in S(1, q)$ we have $\varphi\left(e_{l_{j}}\right)=$ $p^{n_{l_{j}}} e_{l_{j+1}}$, where the integers $n_{l_{j}}$ 's are either all non-negative or all non-positive;

(ii.c) if $v:=\operatorname{dim}\left(G_{B(k)}\right)\left(\operatorname{so} v \in S\left(1, v_{1}\right)\right)$, then the intersection $E:=E_{1} \cap \operatorname{Lie}\left(G_{B(k)}\right)$ has $\left\{e_{1}, \ldots, e_{v}\right\}$ as a $W(k)$-basis and moreover the permutation $\pi_{1}$ normalizes $S(1, v)$;

(ii.d) for any $t \in \mathbf{N}$ and every element of $G(W(k))$ that has the form $1_{M}+\sum_{l \in S\left(1, v_{1}\right)} p^{t} x_{l} e_{l}$, where all $x_{l}$ 's belong to $W(k)$, we have $x_{l} \in p W(k)$ for all $l \in S\left(v+1, v_{1}\right)$.

Let

$$
E_{2}:=E_{1}\left[\frac{1}{p}\right] \cap \operatorname{End}(M)=\operatorname{Lie}\left(G_{1 B(k)}\right) \cap \operatorname{End}(M) .
$$

From (ii.a) we get that $1_{M}+E_{2}$ is a semigroup with identity contained in $\operatorname{End}(M)$.

3.3.1. LemMA. - There exists a closed subgroup scheme $G_{2}$ of $\mathbf{G L}_{M}$ that is defined by the rule: if $A$ is a commutative $W(k)$-algebra, then $G_{2}(A)$ is the group of invertible elements of the semigroup with identity $1_{M \otimes_{W(k)} A}+E_{2} \otimes_{W(k)} A$. We have $G_{1}=G_{2}$.

Proof. - We show that $G_{2}$ is an integral, closed subgroup scheme of $\mathbf{G L}_{M}$ and that $\operatorname{Lie}\left(G_{2 B(k)}\right) \subseteq E_{2}\left[\frac{1}{p}\right]=E_{1}\left[\frac{1}{p}\right]$. If $1_{M} \in E_{2}$, then $1_{M}+E_{2}=E_{2}$ is a $W(k)$-subalgebra of $\operatorname{End}(M)$ which as a $W(k)$-submodule is a direct summand; so obviously $G_{2}$ is an integral, closed subgroup scheme of $\mathbf{G L}_{M}$ and we have $\operatorname{Lie}\left(G_{2 B(k)}\right) \subseteq E_{2}\left[\frac{1}{p}\right]=E_{1}\left[\frac{1}{p}\right]$.

We now consider the case when $1_{M} \notin E_{2}$. Let $E_{3}:=\operatorname{End}(M) \cap\left(E_{2}\left[\frac{1}{p}\right]+B(k) 1_{M}\right)$; it is a $W(k)$-subalgebra of $\operatorname{End}(M)$ that has $E_{2}$ as a two-sided ideal. The finite $W(k)$-algebra $E_{3} / E_{2}$ is isomorphic to a $W(k)$-subalgebra of $B(k) 1_{M}$ and so to $W(k) 1_{M}$. So as $W(k)$-modules, we have a direct sum decomposition $E_{3}=E_{2} \oplus W(k) 1_{M}$. Let $G_{3}$ be the integral, closed subgroup scheme of $\mathbf{G L}_{M}$ of invertible elements of $E_{3}$. Let $x \in 1_{M \otimes_{W(k)} A}+E_{2} \otimes_{W(k)} A$ be an element that has an invertible determinant. The inverse $x^{-1} \in \operatorname{End}(M)$ of $x$ belongs to $G_{3}(A)$ and moreover modulo the ideal $E_{2} \otimes_{W(k)} A$ of $E_{3} \otimes_{W(k)} A$ it is $1_{M \otimes_{W(k)} A}$. Thus $x^{-1} \in 1_{M \otimes_{W(k)} A}+E_{2} \otimes_{W(k)} A$. This implies that the group $G_{2}(A)$ is the group of all elements of $1_{M \otimes_{W(k)} A}+E_{2} \otimes_{W(k)} A$ that have an invertible determinant. From this description of points of $G_{2}$, we get that $G_{2}$ is an integral, closed subgroup scheme of either $\mathbf{G L}_{M}$ or $G_{3}$ and that we have $\operatorname{Lie}\left(G_{2 B(k)}\right) \subseteq E_{2}\left[\frac{1}{p}\right]=E_{1}\left[\frac{1}{p}\right]$.

If $x \in E_{1}$, then we have $1_{M}+p^{t} x \in G_{2}(W(k))$ for all $t \gg 0$. Thus $E_{1}\left[\frac{1}{p}\right] \subseteq \operatorname{Lie}\left(G_{2 B(k)}\right)$ and therefore we have identities $\operatorname{Lie}\left(G_{1 B(k)}\right)=E_{1}\left[\frac{1}{p}\right]=E_{2}\left[\frac{1}{p}\right]=\operatorname{Lie}\left(G_{2 B(k)}\right)$. So $G_{1 B(k)}=$ $G_{2 B(k)}$, cf. [1, Ch. II, 7.1]. Thus $G_{1}=G_{2}$.

3.3.2. TheOREM. - We recall that conditions 3.3(i) and (ii) hold. Let $m_{1} \in \mathbf{N} \cup\{0\}$ be the smallest number such that $p^{m_{1}}\left(E_{2}\right) \subseteq E_{1} \subseteq E_{2}$. Let $g \in G(W(k)) \cap\left(1_{M}+p^{j} E_{1}\right)$ with $j \in \mathbf{N}$. If $p=2$ we assume that either $G=G_{1}$ or $j \geqslant 2$. We have:

(a) There exists $\tilde{g} \in G(W(k)) \cap\left(1_{M}+p^{j} E_{1}\right)$ which is an isomorphism between $(M, g \varphi, G)$ and $(M, \varphi, G)$.

(b) The $i$-number of $(M, \varphi, G)$ is at most $m_{1}+1$.

Proof. - To prove (a), we write $g=1_{M}+\sum_{l \in S\left(1, v_{1}\right)} p^{j} x_{l}(j) e_{l}$, where all $x_{l}(j)$ 's belong to $W(k)$. By induction on $t \in\{j, j+1, \ldots\}$ we construct an element $\tilde{g}_{t} \in G(W(k))$ such that there 
exist elements $x_{l}(t)$ 's in $W(k)$ that satisfy the identity

$$
\tilde{g}_{t} \tilde{g}_{t-1} \cdots \tilde{g}_{j} g \varphi \tilde{g}_{j}^{-1} \cdots \tilde{g}_{t-1}^{-1} \tilde{g}_{t}^{-1} \varphi^{-1}=1_{M}+\sum_{l \in S\left(1, v_{1}\right)} p^{t} x_{l}(t) e_{l} .
$$

If $t=j$ let $\tilde{g}_{t}:=1_{M}$. The passage from $t$ to $t+1$ goes as follows. For $l \in S\left(1, v_{1}\right)$ let $q_{l}:=-\min \left\{0, n_{l}\right\}$; we recall that $n_{l} \in \mathbf{Z}$ is such that $\varphi\left(e_{l}\right)=p^{n_{l}} e_{\pi_{1}(l)}$ (cf. 3.3(ii.b)).

We first consider the case $p \geqslant 3$. Let

$$
\tilde{g}_{t+1}:=\prod_{l \in S(1, v)} \exp \left(p^{t+q_{l}} \tilde{x}_{l}(t+1) e_{l}\right) \in G(W(k))
$$

where all $\tilde{x}_{l}(t+1)$ 's belong to $W(k)$. As $\pi_{1}$ normalizes $S(1, v)$, we have

$$
\varphi \tilde{g}_{t+1}^{-1} \varphi^{-1}=\prod_{l \in S(1, v)} \exp \left(-p^{t+q_{l}+n_{l}} \sigma\left(\tilde{x}_{l}(t+1)\right) e_{\pi_{1}(l)}\right) \in G(W(k))
$$

(to be compared with (14)). As $E_{1} E_{1} \subseteq E_{1}$ and $p \geqslant 3$, for any $e \in E_{1}$ the element $\exp \left(p^{t} e\right)=$ $1_{M}+\sum_{i=1}^{\infty} \frac{p^{t i}}{i !} e^{i}$ belongs to $1_{M}+p^{t} E_{1}$. From this and the inequalities $q_{l} \geqslant 0$ and $q_{l}+n_{l} \geqslant 0$, we get that any exponential element of either (20b) or (20c) belongs to $1_{M}+p^{t} E_{1}$. Thus, as $1_{M}+p^{t} E_{1}$ is a semigroup contained in $\operatorname{End}(M)$, we get that $\tilde{g}_{t+1} \in 1_{M}+p^{t} E_{1}$ and that (cf. also (20a)) we can write

$$
\tilde{g}_{t+1}\left(\tilde{g}_{t} \cdots \tilde{g}_{j} g \varphi \tilde{g}_{j}^{-1} \cdots \tilde{g}_{t}^{-1} \varphi^{-1}\right) \varphi \tilde{g}_{t+1}^{-1} \varphi^{-1}=1_{M}+\sum_{l \in S\left(1, v_{1}\right)} p^{t} x_{l}^{\prime}(t+1) e_{l},
$$

where all $x_{l}^{\prime}(t+1)$ 's belong to $W(k)$. If $i>v$, then $x_{l}^{\prime}(t+1) \in p W(k)$ (cf. 3.3(ii.d)).

Based on 2.6(a), from (20b), (20c), and (20d) we get that for any $l \in S(1, v)$ the Witt vector $x_{l}^{\prime}(t+1) \in W(k)$ is congruent $\bmod p$ to the sum (to be compared with (15))

$$
p^{q_{l}} \tilde{x}_{l}(t+1)+x_{l}(t)-p^{n_{1}^{-1}(l)}+q_{\pi_{1}^{-1}(l)} \sigma\left(\tilde{x}_{\pi_{1}^{-1}(l)}(t+1)\right) .
$$

As in the part of the proof of 3.1.1 that pertains to $(+)$ and $(-)$, we argue that we can choose the $\tilde{x}_{l}(t+1)$ 's with $l \in S(1, v)$ such that we have $x_{l}^{\prime}(t+1) \in p W(k)$ for all $l \in S(1, v)$. Thus for all $l \in S\left(1, v_{1}\right)$ we can write $p^{t} x_{l}^{\prime}(t+1)=p^{t+1} x_{l}(t+1)$, where $x_{l}(t+1) \in W(k)$. This ends the induction for $p \geqslant 3$.

Let now $p=2$. For $t \geqslant 2$, we have $2 t-1 \geqslant t+1$. So the above passage from $t$ to $t+1$ has to be modified only if $t=j=1$, cf. 2.6(b). As $E_{1} E_{1} \subseteq E_{1}$, for any $e \in E_{1}$ the element $1_{M}+$ pe has an inverse in $1_{M}+p E_{1}$ and thus (cf. 3.3.1) it is an element of $G_{2}(W(k))=G_{1}(W(k))$. Thus, if $t=j=1$ and $G=G_{1}$, we can take

$$
\tilde{g}_{2}:=1_{M}+\sum_{l \in S(1, v)} p^{1+q_{l}} \tilde{x}_{l}(2) e_{l} \in G_{2}(W(k))=G_{1}(W(k))=G(W(k))
$$

and we can proceed as above. This ends the induction.

The infinite product $\tilde{g}:=\cdots \tilde{g}_{j+2} \tilde{g}_{j+1} \tilde{g}_{j} \in G(W(k)) \cap\left(1_{M}+p^{j} E_{1}\right)$ is well defined (as $W(k)$ is $p$-adically complete). Passing to limit $t \rightarrow \infty$ in (20a), we get $\tilde{g} g \varphi \tilde{g}^{-1} \varphi^{-1}=1_{M}$. Thus $\tilde{g}$ is an isomorphism between $(M, g \varphi, G)$ and $(M, \varphi, G)$. So (a) holds. 
We prove (b). For $g \in G(W(k)) \leqslant G_{1}(W(k))$, we have $g-1_{M} \in E_{2}$ (cf. 3.3.1). If $g$ is congruent mod $p^{m_{1}+1}$ to $1_{M}$, then $g-1_{M} \in p^{m_{1}+1} E_{2} \subseteq p E_{1}$. Thus $g \in G(W(k)) \cap\left(1_{M}+\right.$ $\left.p E_{1}\right)$. So there exists an isomorphism between $(M, g \varphi, G)$ and $(M, \varphi, G)$ which is an element of $G(W(k))$, cf. (a) applied with $j=1$. So (b) follows from Definition 3.1.4.

\subsubsection{Variant}

Suppose $G=G_{1}$ and $E_{1} E_{1}=0$. Then 3.3.2(a) holds even if $j=0$, i.e. even if we only have $g \in G(W(k)) \cap\left(1_{M}+E\right)$. This is so as we have $\exp (x)=1+x$ for any $x \in E=E_{1}$. Thus the proof of 3.3.2(a) holds even if $j=0$ and so the proof of 3.3.2(b) can be adapted to get that the $i$-number of $(M, \varphi, G)$ is at most $m_{1}$.

3.3.4. COROLlary. - Let $m_{1}$ be as in 3.3.2. We assume that $G=G_{1}$ and that all slopes of $\left(\operatorname{Lie}\left(G_{B(k)}\right), \varphi\right)$ are 0. Then the $i$-number of $(M, \varphi, G)$ is at most $m_{1}$.

Proof. - Let $E_{\mathbf{Z}_{p}}:=\left\{x \in \operatorname{Lie}\left(G_{B(k)}\right) \cap \operatorname{End}(M) \mid \varphi(x)=x\right\}$. We can assume $E=E_{1}$ is the $W(k)$-span of $E_{\mathbf{Z}_{p}}$. We have $E_{\mathbf{Z}_{p}} E_{\mathbf{Z}_{p}} \subseteq E_{\mathbf{Z}_{p}}$, cf. 3.3(ii.a). Let $g_{m_{1}} \in G(W(k))$ be congruent $\bmod p^{m_{1}}$ to $1_{M}$. We write $g_{m_{1}}=1_{M}+e$, with $e \in E$ (to be compared with the proof of 3.3.2(b)). Based on 3.3.2(a), to prove the corollary it suffices to check that there exists $\tilde{g} \in G(W(k))$ such that $\tilde{g} g_{m_{1}} \varphi \tilde{g}^{-1} \varphi^{-1} \in G(W(k)) \cap\left(1_{M}+p E\right)$.

The element $1+e=g_{m_{1}} \in G(W(k))$ normalizes $E_{2}$. Thus $\left(1_{M}+e\right) E \subseteq E \subseteq E_{2}=$ $\left(1_{M}+e\right) E_{2}$. So by reasons of length of artinian modules we get that $\left(1_{M}+e\right) E=E$. Let $e^{\prime} \in E$ be such that $\left(1_{M}+e\right) e^{\prime}=-e$. Thus $\left(1_{M}+e\right)\left(1_{M}+e^{\prime}\right)=1_{M}+e-e=1_{M}$. So $\left(1_{M}+e\right)^{-1}=1_{M}+e^{\prime} \in 1_{M}+E=1_{M}+E_{\mathbf{z}_{p}} \otimes \mathbf{z}_{p} W(k)$.

Let $H$ be the group scheme over $\operatorname{Spec}\left(\mathbf{Z}_{p}\right)$ defined by the rule: if $A$ is a commutative $\mathbf{Z}_{p}$-algebra, then $H(A)$ is the group of invertible elements of the semigroup with identity $1_{M \otimes_{W(k)} A}+E_{\mathbf{Z}_{p}} \otimes_{\mathbf{z}_{p}} A$ contained in $\operatorname{End}(M) \otimes_{W(k)} A$. The automorphism $\sigma$ of $W(k)$ acts naturally on $H(W(k))$. If $u \in H(W(k))$, then $u \in G(W(k))$ (cf. 3.3.1) and $\sigma(u)=\sigma u \sigma^{-1}$ is $\varphi u \varphi^{-1} \in H(W(k))$. Moreover we have $g_{m_{1}} \in H(W(k))$, cf. previous paragraph.

Let $\bar{g}_{m_{1}}$ be the image of $g_{m_{1}}$ in $H(k)$. The scheme $H$ is an open subscheme of the affine space over $\operatorname{Spec}\left(\mathbf{Z}_{p}\right)$ that is of relative dimension $v$ and that is defined naturally by $E_{\mathbf{Z}_{p}}$. Thus the group scheme $H$ over $\operatorname{Spec}\left(\mathbf{Z}_{p}\right)$ has connected fibres and is smooth. Moreover, the special fibre $H_{\mathbf{F}_{p}}$ is a quasi-affine group and thus also an affine group over $\operatorname{Spec}\left(\mathbf{F}_{p}\right)$ (cf. [6, vol. I, Exp. $\left.\left.\mathrm{VI}_{B}, 11.11\right]\right)$. Let $\overline{\tilde{g}} \in H(k)$ be such that $\overline{\tilde{g}} \bar{g}_{m_{1}} \sigma(\overline{\tilde{g}})^{-1}$ is the identity element of $H(k)$, cf. Lang theorem (see $[1, \mathrm{Ch}$. V, 16.4]). Let $\tilde{g} \in H(W(k))$ be an element that lifts $\overline{\tilde{g}}$. The element $\tilde{g} g_{m_{1}} \varphi \tilde{g}^{-1} \varphi^{-1}=\tilde{g} g_{m_{1}} \sigma\left(\tilde{g}^{-1}\right) \in H(W(k)) \leqslant G(W(k))$ has a trivial image in $H(k)$ and thus it belongs to $1_{M}+p E$.

3.3.5. Example. - Let $c \in \mathbf{N}$ be such that g.c.d. $\left(c, r_{M}-c\right)=1$. We assume $(M, \varphi)$ is the Dieudonné module of a $p$-divisible group $D$ over $\operatorname{Spec}(k)$ of height $r:=r_{M}$, dimension $d:=$ $r-c=r_{M}-c$, and (unique) slope $\frac{d}{r}$. Let $m:=\mathbf{T}(\operatorname{End}(M), \varphi)$. Let $E$ be the $W(k)$-subalgebra of $\operatorname{End}(M)$ generated by elements of $\operatorname{End}(M)$ fixed by $\varphi$. As all slopes of $\left(\operatorname{End}\left(M\left[\frac{1}{p}\right]\right), \varphi\right)$ are 0 , any $W(k)$-submodule $O$ of $\operatorname{End}(M)$ with the property that $(O, \varphi)$ is a DieudonnéFontaine $p$-divisible object over $k$, is $W(k)$-generated by elements fixed by $\varphi$ and so is contained in $E$. Thus $m \in \mathbf{N} \cup\{0\}$ is the smallest number such that $p^{m} \operatorname{End}(M) \subseteq E$. As $c \in \mathbf{N}$, we have $E \neq \operatorname{End}(M)$ and so $m \geqslant 1$. The $i$-number $n$ of $(M, \varphi)$ is at most $m$, cf. 3.3.4. But $D$ is uniquely determined up to isomorphism by $D\left[p^{n}\right]$ (cf. 3.2.3) and thus also by $D\left[p^{m}\right]$. Using direct sums of $t \in \mathbf{N}$ copies of $(M, \varphi)$, a similar argument shows that $D^{\mathrm{t}}$ is uniquely determined up to isomorphism by $D^{\mathrm{t}}\left[p^{m}\right]$.

3.3.6. Example. - The case $m=1$ of 3.3 .5 also solves positively the isoclinic case of [35, Conjecture 5.7] as one can easily check this starting from [10, 5.3 and 5.4]. For the reader's

$4^{\text {e }}$ SÉRIE - TOME $39-2006-\mathrm{N}^{\circ} 2$ 
convenience here is the version of the last sentence in the spirit of this paper. In this section, we use the notations of 3.3.5 and we moreover assume that $D$ is such that there exists a $W(k)$-basis $\left\{e_{0}, e_{1}, \ldots, e_{r-1}\right\}$ of $M$ with the property that $\varphi\left(e_{i}\right)$ is $e_{i+d}$ if $i \in S(0, c-1)$ and is $p e_{i+d}$ if $i \in S(c, r-1)$. Here and below, all the lower right indices of the form ${ }_{i}$ and ${ }_{i, j}$ are mod $r$. For $i, j \in S(0, r-1)$ let $e_{i, j} \in \operatorname{End}(M)$ be such that it annihilates $e_{j^{\prime}}$ if $j^{\prime} \neq j$ and takes $e_{j}$ into $e_{i}$. We have $\varphi\left(e_{i, j}\right)=p^{n_{i, j}} e_{i+d, j+d}$, where the integer $n_{i, j}$ is defined by the rule:

(*) it is 0 if $(i, j) \in S(0, c-1)^{2} \cup S(c, r-1)^{2}$, it is 1 if $(i, j) \in S(c, r-1) \times S(0, c-1)$, and it is -1 if $(i, j) \in S(0, c-1) \times S(c, r-1)$.

We check that $m=1$. Replacing $D$ by $D^{\mathrm{t}}$ if necessary, we can assume $c>d=r-c$; so $c>\frac{r}{2}$. Based on (2) and the inequality $m \geqslant 1$, to show that $m=1$ it is enough to show that for all pairs $(i, j) \in S(0, r-1)^{2}$ we have an equality $\mathbf{S} \tau_{i, j}=1$, where $\tau_{i, j}:=$ $\left(n_{i, j}, n_{i+d, j+d}, n_{i+2 d, j+2 d}, \ldots, n_{i+(r-1) d, j+(r-1) d}\right)$.

So it suffices to show that none of the $\tau_{i, j}$ 's is of the form $(-1,0,0, \ldots, 0,-1, \ldots)$, cf. Definitions 2.2.4(b) and (c). Thus it suffices to show that for any pair $\left(i_{0}, j_{0}\right) \in S(0, c-1) \times$ $S(c, r-1)$, the first non-zero number of the sequence $n_{i_{0}+d, j_{0}+d}, \ldots, n_{i_{0}+r d, j_{0}+r d}$ is 1 . We write $j_{0}=c+d_{0}$, with $d_{0} \in S(0, d-1)$. As $d_{0} \in S(0, c-1)$, we can assume $n_{i_{0}+d, j_{0}+d}=n_{i_{0}+d, d_{0}}$ is 0 . Thus $\left(i_{1}, j_{1}\right):=\left(i_{0}+d, d_{0}\right) \in S(0, c-1)^{2}$ and we have $i_{1} \geqslant j_{1}$.

We have to show that the first non-zero number of the sequence $n_{i_{1}, j_{1}}, n_{i_{1}+d, j_{1}+d}, \ldots$, $n_{i_{1}+(r-1) d, j_{1}+(r-1) d}$ is 1 . We can assume $n_{i_{1}+d, j_{1}+d} \neq 1$. As $i_{1} \geqslant j_{1}$ we have $n_{i_{1}+d, j_{1}+d} \neq-1$. Thus $n_{i_{1}+d, j_{1}+d}=0$. If $i_{1}+d \leqslant c-1$, let $\left(i_{2}, j_{2}\right):=\left(i_{1}+d, j_{1}+d\right) \in S(0, c-1)^{2}$; if $i_{1}+d \geqslant c$, then from $(*)$ and the equality $n_{i_{1}+d, j_{1}+d}=0$ we get $j_{1}+d \geqslant c$ and thus we have $\left(i_{2}, j_{2}\right):=\left(i_{1}+2 d-r, j_{1}+2 d-r\right) \in S(0, c-1)^{2}$. We conclude that $\left(i_{2}, j_{2}\right) \in S(0, c-1)^{2}$ and $i_{2} \geqslant j_{2}$. We have $n_{i_{2}, j_{2}}=0$. We have to show that the first non-zero number of the sequence $n_{i_{2}, j_{2}}, \ldots, n_{i_{2}+(r-1) d, j_{2}+(r-1) d}$ is 1 . As in this way we cannot construct indefinitely pairs $\left(i_{u}, j_{u}\right) \in S(0, c-1)^{2}$ with $i_{u} \geqslant j_{u}$ (here $u \in \mathbf{N}$ ), we get that the first non-zero number of the sequence $n_{i_{2}, j_{2}}, \ldots, n_{i_{2}+(r-1) d, j_{2}+(r-1) d}$ is 1 .

So $\mathbf{S} \tau_{i, j}=1$ and $m=1$. Thus for $t \in \mathbf{N}, D^{\mathrm{t}}$ is uniquely determined up to isomorphism by $D^{\mathrm{t}}[p]$ (cf. end of 3.3.5). This was predicted by [35, Conjecture 5.7].

3.3.7. Example. - We assume that all slopes of $(\operatorname{End}(M), \varphi)$ are 0 and that $G_{1}=\mathbf{G L}_{M}$. Thus 3.3(i) holds. Let $E_{1} \mathbf{Z}_{p}$ be the $\mathbf{Z}_{p}$-subalgebra of $\operatorname{End}(M)$ formed by elements fixed by $\varphi$. Let $E_{1}:=E_{1} \mathbf{Z}_{p} \otimes \mathbf{z}_{p} W(k) \subseteq \operatorname{End}(M)$; we have $v_{1}=r_{M}^{2}$ and $E_{2}=\operatorname{End}(M)$. We also assume that $p \geqslant 3$ and that $G=\mathbf{S p}\left(M, \lambda_{M}\right)$ (respectively and that $G=\mathbf{S O}\left(M, \lambda_{M}\right)$ ), where $\lambda_{M}$ is a perfect alternating (respectively perfect symmetric) bilinear form on $M$ which is a principal (respectively a principal bilinear) quasi-polarization of $(M, \varphi)$. As $p \geqslant 3$, we have a direct sum decomposition $\operatorname{End}(M)=\operatorname{Lie}(G) \oplus \operatorname{Lie}(G)^{\perp}$, where $\operatorname{Lie}(G)^{\perp}$ is the perpendicular of $\operatorname{Lie}(G)$ with respect to the trace form on $\operatorname{End}(M)$. As $\varphi$ normalizes $\operatorname{Lie}(G)\left[\frac{1}{p}\right]$, it also normalizes $\operatorname{Lie}(G)^{\perp}\left[\frac{1}{p}\right]$. Thus $E_{1}$ has a $W(k)$-basis $\mathcal{B}_{1}=\left\{e_{1}, \ldots, e_{v_{1}}\right\}$ that is the disjoint union of a $\mathbf{Z}_{p}$-basis $\left\{e_{1}, \ldots, e_{v}\right\}$ of $E_{1} \mathbf{z}_{p} \cap \operatorname{Lie}(G)$ and of a $\mathbf{Z}_{p}$-basis $\left\{e_{v+1}, \ldots, e_{v_{1}}\right\}$ of $E_{1 \mathbf{Z}_{p}} \cap \operatorname{Lie}(G)^{\perp}$. Let $\pi_{1}:=1_{S\left(1, v_{1}\right)}$.

Properties 3.3(ii.a) to (ii.c) hold, cf. constructions. We check that 3.3(ii.d) holds. Let $g \in$ $G(W(k))$ be of the form $1_{M}+\sum_{l \in S\left(1, v_{1}\right)} p^{t} x_{l} e_{l}$, where all $x_{l}$ 's belong to $W(k)$. The involution of $\operatorname{End}(M)$ defined by $\lambda_{M}$ fixes $\operatorname{Lie}(G)^{\perp}$ and acts as -1 on $\operatorname{Lie}(G)$. Thus the product $\left(1_{M}-\sum_{l=1}^{v} p^{t} x_{l} e_{l}+\sum_{l=v+1}^{v_{1}} p^{t} x_{l} e_{l}\right)\left(1_{M}+\sum_{l \in S\left(1, v_{1}\right)} p^{t} x_{l} e_{l}\right)$ is $1_{M}($ as $g \in G(W(k)))$ and belongs to $1_{M}+2\left(\sum_{l=v+1}^{v_{1}} p^{t} x_{l} e_{l}\right)+p^{t+1} E_{1}$. As $p \geqslant 3$, for $l \in S\left(v+1, v_{1}\right)$ we have $x_{l} \in$ $p W(k)$. So 3.3(ii.d) holds. Thus 3.3(ii) holds. So 3.3.2 applies. In particular, the $i$-number of $(M, \varphi, G)$ is at most $m_{1}+1$, where $m_{1} \in \mathbf{N} \cup\{0\}$ is the smallest number such that we have $p^{m_{1}} \operatorname{End}(M) \subseteq E_{1}=E_{1 \mathbf{z}_{p}} \otimes \mathbf{z}_{p} W(k)$ (cf. 3.3.2(b)). 


\section{Four examples}

In this section we include four examples that pertain to Sections 3.1 to 3.3. Let $\varepsilon_{p} \in\{1,2\}$ be as before 3.1. Let $(M, \varphi, G)$ be a $p$-divisible object with a group over $k$. Let $n \in \mathbf{N} \cup\{0\}$ be the $i$-number of $(M, \varphi, G)$, cf. 3.1.4. In this section we will assume that $k=\bar{k}$ and that $G$ is a reductive group scheme over $\operatorname{Spec}(W(k))$. Thus the group scheme $G$ is smooth over $\operatorname{Spec}(W(k))$ and its fibres are connected and have trivial unipotent radicals. As $G$ is smooth over $\operatorname{Spec}(W(k))$, with the notations of 2.7 we have $G=G^{\prime}$ and $n(G)=0$. Let $M=\bigoplus_{i=a}^{b} \widetilde{F}^{i}(M)$, $\left(F^{i}(M)\right)_{i \in S(a, b)}$, and $\mu: \mathbf{G}_{m} \rightarrow G$ be as in 2.2.1(d). Let $b_{L} \in S(0, b-a)$ be the smallest number such that we have a direct sum decomposition

$$
\operatorname{Lie}(G):=\bigoplus_{i=-b_{L}}^{b_{L}} \widetilde{F}^{i}(\operatorname{Lie}(G))
$$

with the property that $\beta \in \mathbf{G}_{m}(W(k))$ acts on $\widetilde{F}^{i}(\operatorname{Lie}(G))$ through $\mu$ as the multiplication with $\beta^{-i}$. As the group scheme $G$ is reductive, both $W(k)$-modules $\widetilde{F}^{b_{L}}(\operatorname{Lie}(G))$ and $\widetilde{F}^{-b_{L}}(\operatorname{Lie}(G))$ are non-zero. As in 2.5, we have a $\sigma$-linear automorphism $\sigma_{0}:=\varphi \mu(p)$ of $M$. As $\varphi=\sigma_{0} \mu\left(p^{-1}\right)$, the $s$-number of $(\operatorname{Lie}(G), \varphi)$ is $b_{L}$. If $b_{L} \leqslant 1$, we say $(M, \varphi, G)$ is a Shimura p-divisible object over $k$. Let $f_{-1} \in \mathbf{N} \cup\{0\}$ be the rank of $\widetilde{F}^{-1}(\operatorname{Lie}(G))$.

In Sections 4.1-4.5 we will consider four unrelated situations.

\subsection{Example 1}

In this section we assume that $b_{L}=1$ and that all slopes of $(\operatorname{Lie}(G), \varphi)$ are 0 . Let $f \in \mathbf{N}$ be the smallest number such that there exists a filtration

$$
0=\mathcal{E}_{0} \subseteq \mathcal{E}_{1} \subseteq \cdots \subseteq \mathcal{E}_{f}:=\operatorname{Lie}(G)
$$

by $W(k)$-submodules that are direct summands, with the property that for any number $i \in$ $S(1, f)$, the quotient $W(k)$-module $\mathcal{E}_{i} / \mathcal{E}_{i-1}$ is a maximal direct summand of $\operatorname{Lie}(G) / \mathcal{E}_{i-1}$ normalized by $\varphi$. For $i \in S(2, f)$ we choose $x_{i} \in \mathcal{E}_{i} \backslash\left(\mathcal{E}_{i-1}+p \mathcal{E}_{i}\right)$ such that we have $p \varphi\left(x_{i}\right)-p x_{i} \in \mathcal{E}_{i-1} \backslash p \mathcal{E}_{i-1}$ and the images of $x_{1}, \ldots, x_{i-1}$, and $x_{i}$ in

$$
\operatorname{Lie}(G) /\left(\widetilde{F}^{0}(\operatorname{Lie}(G))+\widetilde{F}^{1}(\operatorname{Lie}(G))+p \operatorname{Lie}(G)\right) \stackrel{\sim}{\longrightarrow} \widetilde{F}^{-1}(\operatorname{Lie}(G)) / p \widetilde{F}^{-1}(\operatorname{Lie}(G))
$$

are $k$-linearly independent. The possibility of making such choices is implied by the maximal property of $\mathcal{E}_{i} / \mathcal{E}_{i-1}$. By reasons of ranks we get $f-1 \leqslant f_{-1}$. By induction on $j \in S(1, f)$ we get $\varphi$ normalizes $\mathcal{E}_{1}+p \mathcal{E}_{2}+\cdots+p^{j-1} \mathcal{E}_{j}$. Taking $j=f$, we get that $E:=\mathcal{E}_{1}+p \mathcal{E}_{2}+\cdots+p^{f-1} \mathcal{E}_{f}$ has a $W(k)$-basis formed by elements fixed by $\varphi$. Thus $(E, \varphi)$ is a Dieudonné $p$-divisible object over $k$. As $p^{f-1}$ annihilates $\operatorname{Lie}(G) / E$ we get $\mathbf{T}(\operatorname{Lie}(G), \varphi) \leqslant f-1$. Thus (cf. 3.1 .5 for the first inequality)

$$
n \leqslant 2 \mathbf{T}(\operatorname{Lie}(G), \varphi)+\varepsilon_{p} \leqslant 2(f-1)+\varepsilon_{p} \leqslant 2 f_{-1}+\varepsilon_{p} .
$$

Often 3.3.4 provides (respectively 3.3.2(b) and 3.3.7 applied with $G_{1}=\mathbf{G L}_{M}$ provide) better upper bounds of $n$. For instance, if $G=\mathbf{G L}_{M}$ (respectively if $p \geqslant 3$ and $G=\mathbf{S p}\left(M, \lambda_{M}\right)$ with $\lambda_{M}$ as a principal quasi-polarization of $(M, \varphi)$ ) we get $n \leqslant \mathbf{T}(\operatorname{Lie}(G), \varphi) \leqslant f_{-1}$ (respectively $\left.n \leqslant \mathbf{T}(\operatorname{Lie}(G), \varphi)+1 \leqslant f_{-1}+1\right)$. If $(M, \varphi)$ is the Dieudonné module of a supersingular $p$-divisible group over $\operatorname{Spec}(k)$ of height $2 d$ and if $G=\mathbf{G L}_{M}$ (respectively and if $p \geqslant 3$ and $G=\mathbf{S p}\left(M, \lambda_{M}\right)$ ), then $f_{-1}$ is $d^{2}$ (respectively is $\frac{d^{2}+d}{2}$ ). Thus we have the following concrete application of 3.2.3 (respectively of 3.2.5):

$4^{\text {e }}$ SÉRIE - TOME $39-2006-\mathrm{N}^{\circ} 2$ 
4.1.1. Proposition. - Let $d \in \mathbf{N}$. If $p \geqslant 2$ (respectively if $p \geqslant 3$ ), then any (respectively any principally quasi-polarized) supersingular p-divisible group of height $r=2 d$ over $\operatorname{Spec}(k)$ is uniquely determined up to isomorphism by its truncated (respectively its principally quasipolarized truncated) Barsotti-Tate group of level $d^{2}$ (respectively of level $\frac{d^{2}+d}{2}+1$ ).

\subsection{Root decompositions}

The image of $\mu$ is either trivial or a closed $\mathbf{G}_{m}$ subgroup of $G$ and thus its centralizer in $G$ is a reductive group scheme which has a maximal torus (cf. [6, vol. III, Exp. XIX, 2.8 and 6.1]). Thus there exists a maximal torus $T$ of $G$ through which $\mu$ factors. We have $\operatorname{Lie}(T) \subseteq \widetilde{F}^{0}(\operatorname{Lie}(G))$. It is easy to check that there exists $g \in G(W(k))$ such that $g \varphi$ normalizes $\operatorname{Lie}(T)$. Accordingly, for the next three examples (i.e. until Section 5) we will assume that we have $\varphi(\operatorname{Lie}(T))=\operatorname{Lie}(T)$. Let

$$
\operatorname{Lie}(G)=\operatorname{Lie}(T) \bigoplus_{\gamma \in \Phi} \mathfrak{g}_{\gamma}
$$

be the root decomposition relative to $T$. So $\Phi$ is a root system of characters of $T$ and each $\mathfrak{g}_{\gamma}$ is a free $W(k)$-module of rank 1 on which $T$ acts via the character $\gamma$.

Let $\Delta$ be a basis of $\Phi$ such that $\bigoplus_{\gamma \in \Delta} \mathfrak{g}_{\gamma} \subseteq \bigoplus_{i=0}^{b_{L}} \widetilde{F}^{i}(\operatorname{Lie}(G))$. Let $\Phi^{+}$and $\Phi^{-}$be the sets of positive and negative (respectively) roots of $\Phi$ with respect to $\Delta$. Let $C$ be the unique Borel subgroup scheme of $G$ which contains $T$ and for which we have $\operatorname{Lie}(C)=$ $\operatorname{Lie}(T) \bigoplus_{\gamma \in \Phi^{+}} \mathfrak{g}_{\gamma}$, cf. [6, vol. III, Exp. XXII, 5.5.1]. As $\operatorname{Lie}(C)\left[\frac{1}{p}\right]$ is generated by the $B(k)-$ vector subspace $\bigoplus_{\gamma \in \Delta} \mathfrak{g}_{\gamma}\left[\frac{1}{p}\right]$ of the Lie algebra $\bigoplus_{i=0}^{b_{L}} \widetilde{F}^{i}(\operatorname{Lie}(G))\left[\frac{1}{p}\right]$, we have an inclusion $\operatorname{Lie}(C) \subseteq \bigoplus_{i=0}^{b_{L}} \widetilde{F}^{i}(\operatorname{Lie}(G))$.

As $\mu$ factors through $T$, for any root $\gamma \in \Phi$ there exists an integer $n(\gamma) \in S\left(-b_{L}, b_{L}\right)$ such that we have $\mathfrak{g}_{\gamma} \subseteq \widetilde{F}^{n(\gamma)}(\operatorname{Lie}(G))$. As $\varphi=\sigma_{0} \mu\left(p^{-1}\right)$ and $\sigma_{0}(\operatorname{Lie}(T))=\varphi(\operatorname{Lie}(T))=\operatorname{Lie}(T)$, there exists a permutation $\Pi$ of $\Phi$ such that we have

$$
\sigma_{0}\left(\mathfrak{g}_{\gamma}\right)=\mathfrak{g}_{\Pi(\gamma)} \quad \text { and } \quad \varphi\left(\mathfrak{g}_{\gamma}\right)=p^{n(\gamma)} \mathfrak{g}_{\Pi(\gamma)}, \quad \forall \gamma \in \Phi
$$

If $\gamma \in \Phi^{+}$(respectively if $\gamma \in \Phi^{-}$), then $n(\gamma) \in S\left(0, b_{L}\right)$ (respectively then $n(\gamma) \in S\left(-b_{L}, 0\right)$ ); this is a consequence of the inclusion $\operatorname{Lie}(C) \subseteq \bigoplus_{i=0}^{b_{L}} \widetilde{F}^{i}(\operatorname{Lie}(G))$.

As $\operatorname{Lie}(T)$ is normalized by $\varphi$, it has a $W(k)$-basis formed by elements fixed by $\varphi$. Let $\Pi_{0}:=\left(\gamma_{1}, \ldots, \gamma_{l}\right)$ be a cycle of $\Pi$. For $j \in S(1, l)$ let $y_{\gamma_{j}} \in \mathfrak{g}_{\gamma_{j}} \backslash\{0\}$ be such that (cf. (21)) we have $\varphi\left(y_{\gamma_{j}}\right)=p^{m\left(\gamma_{j}\right)} y_{\gamma_{j+1}}$ (with $\left.\gamma_{l+1}:=\gamma_{1}\right)$, where $m\left(\gamma_{1}\right), \ldots, m\left(\gamma_{l}\right)$ are integers that are either all positive or all negative. Let $\mathcal{B}_{0}:=\left\{y_{\gamma_{1}}, \ldots, y_{\gamma_{l}}\right\}$.

Let $E$ be a $W(k)$-submodule of $\operatorname{Lie}(G)$ that contains $\operatorname{Lie}(T)$, that satisfies the identity $E\left[\frac{1}{p}\right]=\operatorname{Lie}(G)\left[\frac{1}{p}\right]$, and that is maximal subject to the property that it has a $W(k)$-basis $\mathcal{B}$ which is the union of a $\mathbf{Z}_{p}$-basis of $\{x \in \operatorname{Lie}(T) \mid \varphi(x)=x\}$ and of subsets $\mathcal{B}_{0}$ that are associated as above to some cycle $\Pi_{0}$ of $\Pi$. Let $\pi$ be the permutation of $\mathcal{B}$ which fixes $\mathcal{B} \cap \operatorname{Lie}(T)$ and which for $\gamma \in \Phi$ takes the element of $\mathfrak{g}_{\gamma} \cap \mathcal{B}$ into the element of $\mathfrak{g}_{\Pi(\gamma)} \cap \mathcal{B}$.

\subsection{Example 2}

It is not difficult to check that there exists an element $g \in G(W(k))$ which normalizes $T$ and which has the property that $g \varphi$ takes $\operatorname{Lie}(C)$ into $\operatorname{Lie}(C)$. Accordingly, in this section we assume that there exists a Borel subgroup $\widetilde{C}$ of $G$ which contains $T$ and which has the property that $\varphi(\operatorname{Lie}(\widetilde{C})) \subseteq \operatorname{Lie}(\widetilde{C})$. So if we have $\mathfrak{g}_{\gamma} \subseteq \operatorname{Lie}(\widetilde{C})$, then $n(\gamma) \geqslant 0$. Thus we have 
$\operatorname{Lie}(\widetilde{C}) \subseteq \bigoplus_{i=0}^{b_{L}} \widetilde{F}^{i}(\operatorname{Lie}(G))$. Thus, not to introduce extra notations, we can assume that $C=\widetilde{C}$; so $\varphi(\operatorname{Lie}(C)) \subseteq \operatorname{Lie}(C)$.

The last inclusion implies that $\Pi$ normalizes both $\Phi^{+}$and $\Phi^{-}$. As we have $n(\gamma) \geqslant 0$ if $\gamma \in \Phi^{+}$ and $n(\gamma) \leqslant 0$ if $\gamma \in \Phi^{-}$, for any cycle $\Pi_{0}=\left(\gamma_{1}, \ldots, \gamma_{l}\right)$ of $\Pi$ we can choose the above elements $y_{\gamma_{j}} \in \mathfrak{g}_{\gamma_{j}}$ to be generators of $\mathfrak{g}_{\gamma_{j}}$. Thus, due to the maximal property of $E$, we have $E=\operatorname{Lie}(G)$ (i.e. for any $\gamma \in \Phi$ the intersection $\mathcal{B} \cap \mathfrak{g}_{\gamma}$ is a $W(k)$-basis of $\left.\mathfrak{g}_{\gamma}\right)$. Thus $\mathbf{T}(\operatorname{Lie}(G), \varphi)=0$, i.e. $(\operatorname{Lie}(G), \varphi)=(E, \varphi)$ is a Dieudonné-Fontaine $p$-divisible object over $k$. We have $n \leqslant \varepsilon_{p}$, cf. 3.1.5. Thus $n \leqslant 1$ if $p \geqslant 3$ and $n \leqslant 2$ if $p=2$.

4.3.1. Proposition. - We recall that $G$ is a reductive group scheme, that $T$ is a maximal torus of $G$ through which $\mu: \mathbf{G}_{m} \rightarrow G$ factors and whose Lie algebra is normalized by $\varphi$, and that we have $\varphi(\operatorname{Lie}(C)) \subseteq \operatorname{Lie}(C)$ for some Borel subgroup scheme $C$ of $G$ that contains $T$. Then the $i$-number $n$ of $(M, \varphi, G)$ is at most 1 .

Proof. - We know that $n \leqslant 1$ if $p \geqslant 3$. Thus we can assume $p=2$ (but as the below arguments work for all primes, we will keep the notation $p$ instead of 2). Let $\Phi(0):=\Phi \cup\{0\}, n(0):=0$, and $G(0):=T$. For $\gamma \in \Phi$ let $G(\gamma)$ be the unique $\mathbf{G}_{a}$ subgroup scheme of $G$ that is normalized by $T$ and such that $\operatorname{Lie}(G(\gamma))=\mathfrak{g}_{\gamma}$, cf. [6, vol. III, Exp. XXII, 1.1]. If $x \in \mathfrak{g}_{\gamma}$ is such that $x^{p} \neq 0$, then the torus $T$ acts on $\left\langle x^{p}\right\rangle$ via the $p$-th power of the character $\gamma$. The reduction $\bar{x}^{p} \bmod p$ of $x^{p}$ belongs to $\mathfrak{g}_{\gamma} \bmod p$, cf. [1, Ch. II, 3.1, 3.5, Lemma 3 of 3.19]. From the last two sentences we get that $\bar{x}^{p}=0$. This implies that for each $\gamma \in \Phi$ we have a bijection $\exp _{\gamma}: \mathfrak{g}_{\gamma} \stackrel{\sim}{\longrightarrow} G(\gamma)(W(k))$ which maps $x \in \mathfrak{g}_{\gamma}$ into $\exp _{\gamma}(x)=\sum_{i=0}^{\infty} \frac{x^{i}}{i !}$ (we emphasize that $\mathfrak{g}_{\gamma}$ is not included in the domain of the exponential map of 2.6 defined for $O=M$ ).

Let $g_{1} \in G(W(k))$ be congruent $\bmod p$ to $1_{M}$. Let $l_{1} \in \operatorname{Lie}(G)$ be such that $g_{1}$ is congruent $\bmod p^{2}$ to $1_{M}+p l_{1}$. We show that there exists $g \in G(W(k))$ congruent $\bmod p$ to $1_{M}$ and such that $g g_{1} \varphi g^{-1} \varphi^{-1} \in G(W(k))$ is congruent $\bmod p^{2}$ to $1_{M}$.

We take $g$ to be a product $\prod_{\gamma \in \Phi(0)} g_{1}^{\gamma}$ (taken in any order), where $g_{1}^{\gamma} \in G(\gamma)(W(k))$ is congruent $\bmod p^{1+\max \{0,-n(\gamma)\}}$ to $1_{M}$. Let $l_{1}^{0} \in \operatorname{Lie}(T)$ be such that $g_{1}^{0} \in G(0)(W(k))$ is congruent $\bmod p^{2}$ to $1_{M}+p l_{1}^{0}$. The element $\varphi g_{1}^{0} \varphi^{-1}=\sigma_{0} g_{1}^{0} \sigma_{0}^{-1} \in G(0)(B(k)) \cap$ $\mathbf{G L}_{M}(W(k))=G(0)(W(k))$ is congruent $\bmod p^{2}$ to $1_{M}+p \sigma_{0}\left(l_{1}^{0}\right)$. For $\gamma \in \Phi$ let $x_{1}^{\gamma} \in$ $p^{1+\max \{0,-n(\gamma)\}} \mathfrak{g}_{\gamma}$ and $l_{1}^{\gamma} \in \mathfrak{g}_{\gamma}$ be such that $g_{1}^{\gamma}=\exp _{\gamma}\left(x_{1}^{\gamma}\right)$ is congruent $\bmod p^{2+\max \{0,-n(\gamma)\}}$ to $1_{M}+p^{1+\max \{0,-n(\gamma)\}} l_{1}^{\gamma}$, cf. 2.6.1 and 2.6.2. Based on (21) we have

$$
\varphi g_{1}^{\gamma} \varphi^{-1}=\exp _{\Pi(\gamma)}\left(p^{1+\max \{0, n(\gamma)\}} \sigma_{0}\left(x_{1}^{\gamma}\right)\right) \in G(\Pi(\gamma))(W(k)) .
$$

So if $n(\gamma)>0$ (respectively if $n(\gamma) \leqslant 0$ ), then from 2.6(b) (respectively from the very definition of $l_{1}^{\gamma}$ ) we get that $\varphi g_{1}^{\gamma} \varphi^{-1}=\exp _{\Pi(\gamma)}\left(p^{1+\max \{0, n(\gamma)\}} \sigma_{0}\left(x_{1}^{\gamma}\right)\right)$ is congruent $\bmod p^{2}$ to $1_{M}$ (respectively to $1_{M}+p \sigma_{0}\left(l_{1}^{\gamma}\right)$ ). Thus by replacing $g_{1}$ with the following product

$$
g g_{1} \varphi g^{-1} \varphi^{-1}=\left(\prod_{\gamma \in \Phi(0)} g_{1}^{\gamma}\right) g_{1}\left(\prod_{\gamma \in \Phi(0)} \varphi g_{1}^{\gamma} \varphi^{-1}\right)^{-1} \in G(W(k))
$$

of elements congruent $\bmod p$ to $1_{M}$, the role of $l_{1}$ gets replaced by the one of

$$
\tilde{l}_{1}:=l_{1}+\sum_{\gamma \in \Phi, n(\gamma)>0} l_{1}^{\gamma}+\sum_{\gamma \in \Phi(0), n(\gamma)=0}\left[l_{1}^{\gamma}-\sigma_{0}\left(l_{1}^{\gamma}\right)\right]+\sum_{\gamma \in \Phi, n(\gamma)<0}-\sigma_{0}\left(l_{1}^{\gamma}\right) .
$$

By writing all elements defining $\tilde{l}_{1}$ as linear combinations of elements of the $W(k)$-basis $\mathcal{B}$ of $E=\operatorname{Lie}(G)$, as in the part of 3.1.1 that involves (+) and (-) we argue that we can choose the 
$l_{1}^{\gamma}$ 's and so also the $g_{1}^{\gamma}$ 's, such that we have $\tilde{l}_{1} \in p \operatorname{Lie}(G)$; here $\gamma \in \Phi(0)$. Thus $g g_{1} \varphi g^{-1} \varphi^{-1}$ is congruent $\bmod p^{2}$ to $1_{M}$. So if $n=2$, then the $i$-number of $(M, \varphi, G)$ is at most 1 and this contradicts the definition of $n$. As $n \leqslant 2$, we get $n \leqslant 1$.

4.3.2. Proposition. - We continue to work under the hypotheses of 4.3.1. Let $E_{0}:=$ $\{e \in \operatorname{Lie}(G) \mid \varphi(e)=e\}$. We have:

(a) The cocharacter $\mu: \mathbf{G}_{m} \rightarrow G$ is the unique Hodge cocharacter of $(M, \varphi, G)$ that centralizes $E_{0}$.

(b) The lift $\left(F^{i}(M)\right)_{i \in S(a, b)}$ is the unique lift of $(M, \varphi, G)$ such that for any $e \in E_{0}$ and every $i \in S(a, b)$ we have $e\left(F^{i}(M)\right) \subseteq F^{i}(M)$.

Proof. - As $\varphi=\sigma_{0} \mu\left(p^{-1}\right)$, we have $n(\gamma)=0$ if and only if $\mathfrak{g}_{\gamma} \subseteq \widetilde{F}^{0}(\operatorname{Lie}(G))$. So as $\Pi$ normalizes $\Phi^{+}$and $\Phi^{-}$and as $n(\gamma) \geqslant 0$ (respectively $n(\gamma) \leqslant 0$ ) if $\gamma \in \Phi^{+}$(respectively if $\left.\gamma \in \Phi^{-}\right)$, we easily get that $E_{0} \subseteq \widetilde{F}^{0}(\operatorname{Lie}(G))$. Thus $\mu$ centralizes $E_{0}$ and for $e \in E_{0}$ we have $e\left(\widetilde{F}^{i}(M)\right) \subseteq \widetilde{F}^{i}(M)$ for all $i \in S(a, b)$; thus $e\left(F^{i}(M)\right) \subseteq F^{i}(M)$ for all $i \in S(a, b)$.

Let $\mu_{1}$ be another Hodge cocharacter of $(M, \varphi, G)$ that centralizes $E_{0}$. As $\varphi(\operatorname{Lie}(T))=$ $\operatorname{Lie}(T), \operatorname{Lie}(T)$ is $W(k)$-generated by elements of $E_{0} \cap \operatorname{Lie}(T)$. Thus $\mu_{1}$ centralizes $\operatorname{Lie}(T)$ and therefore it factors through $T$. So $\mu$ and $\mu_{1}$ commute. So to show that $\mu=\mu_{1}$ it is enough to show that $\mu_{k}=\mu_{1 k}$. As $\varphi^{-1}(M)=\left(\sigma_{0} \mu\left(p^{-1}\right)\right)^{-1}(M)=\bigoplus_{i=a}^{b} p^{-i} \widetilde{F}^{i}(M)$, for $i \in S(a, b)$ we have $\left(\left(p^{-i} \varphi\right)^{-1}(M)\right) \cap M=\sum_{t=0}^{i-a} p^{t} F^{i-t}(M)$. This identity implies that the filtration $\left(F^{i}(M) / p F^{i}(M)\right)_{i \in S(a, b)}$ of $M / p M$ is uniquely determined by $(M, \varphi)$. Thus both cocharacters $\mu_{k}$ and $\mu_{1 k}$ act on $F^{i}(M) / F^{i+1}(M)+p F^{i}(M) \stackrel{\sim}{\longrightarrow} \widetilde{F}^{i}(M) / p \widetilde{F}^{i}(M)$ via the $-i$-th power of the identity character of $\mathbf{G}_{m}$. So as $\mu_{k}$ and $\mu_{1 k}$ commute, by decreasing induction on $i \in S(a, b)$ we get that $\widetilde{F}^{i}(M) / p \widetilde{F}^{i}(M)$ is the maximal $k$-vector subspace of $M / p M$ on which both $\mu_{k}$ and $\mu_{1 k}$ act via the $-i$-th power of the identity character of $\mathbf{G}_{m}$. This implies $\mu_{k}=\mu_{1 k}$. Thus $\mu=\mu_{1}$. So (a) holds.

Let $\left(F_{1}^{i}(M)\right)_{i \in S(a, b)}$ be another lift of $(M, \varphi, G)$ such that for any $e \in E_{0}$ and $i \in$ $S(a, b)$, we have $e\left(F_{1}^{i}(M)\right) \subseteq F_{1}^{i}(M)$. The inverse of the canonical split cocharacter of $\left(M,\left(F_{1}^{i}(M)\right)_{i \in S(a, b)}, \varphi\right)$ fixes all elements of $E_{0}$ (cf. the functorial aspects of [42, p. 513]), factors through $G$ (cf. 2.5), and thus it is $\mu$ (cf. (a)). Thus for $i \in S(a, b)$ we have $F^{i}(M)=$ $\bigoplus_{j=i}^{b} \widetilde{F}^{j}(M)=F_{1}^{i}(M)$. So (b) holds.

If $g \in G(W(k))$, then the Newton polygon of $(M, g \varphi)$ is above the Newton polygon of $(M, \varphi)$ (cf. [37, Thm. 4.2]). Thus Proposition 4.3.1 generalizes the well known fact that an ordinary $p$-divisible group $D$ over $\operatorname{Spec}(k)$ is uniquely determined up to isomorphism by $D[p]$. Proposition 4.3.2 generalizes the well known fact that the canonical lift of $D$ is the unique lift of $D$ to $\operatorname{Spec}(W(k))$ with the property that any endomorphism of $D$ lifts to it. The last two sentences motivate the next definition.

4.3.3. Definition. - We refer to $(M, \varphi, G)$ of 4.3 .1 as an ordinary p-divisible object with a reductive group over $k$ and to either $\left(F^{i}(M)\right)_{i \in S(a, b)}$ or $\left(M,\left(F^{i}(M)\right)_{i \in S(a, b)}, \varphi, G\right)$ as the canonical lift of $(M, \varphi, G)$.

\subsection{Example 3}

Let $c \in \mathbf{N}$. We assume that $b_{L}=1$ and that there exists a direct sum decomposition $M=$ $\bigoplus_{i=1}^{c} M_{i}$ in $W(k)$-modules of rank 2 such that $G=\prod_{i=1}^{c} \mathbf{G L}_{M_{i}}$ and we have $\varphi\left(M_{i}\left[\frac{1}{p}\right]\right)=$ $M_{i+1}\left[\frac{1}{p}\right]$ for $i \in S(1, c)$, where $M_{c+1}:=M_{1}$. We have $r_{M}=2 c$. As $b_{L}=1$ and $G \stackrel{\sim}{\longrightarrow} \mathbf{G L}_{2}^{c}$, we have $f_{-1} \in S(1, c)$. For $i \in S(1, c)$ we have $\varphi\left(\operatorname{End}\left(M_{i}\right)\left[\frac{1}{p}\right]\right)=\operatorname{End}\left(M_{i+1}\right)\left[\frac{1}{p}\right]$. Thus the permutation $\pi$ of $\mathcal{B}$ has at most two cycles formed by elements of $\mathcal{B} \backslash \operatorname{Lie}(T)$ (equivalently the 
permutation $\Pi$ of $\Phi$ has at most two cycles). If we have one such cycle, then its length is $2 c$. If we have two such cycles, then their length is $c$.

Let $\varepsilon \in\{1,2\}$. Let $l=\varepsilon c$ be such that we have a cycle $\left(y_{1}, \ldots, y_{l}\right)$ of $\pi$ formed by elements of $\mathcal{B} \backslash \operatorname{Lie}(T)$. For $i \in S(1, l)$ let $s_{i} \in \mathbf{N} \cup\{0\}$ be such that $e_{i}:=p^{-s_{i}} y_{i}$ generates $\mathfrak{g}_{\gamma_{i}}$ for some $\gamma_{i} \in \Phi$. Let $\left(n_{1}, \ldots, n_{l}\right):=\left(n\left(\gamma_{1}\right), \ldots, n\left(\gamma_{l}\right)\right) \in \mathbf{Z}^{l}$; we have $\varphi\left(e_{i}\right)=p^{n_{i}} e_{i+1}$ (cf. (21)). As $b_{L}=1$, for $i \in S(1, l)$ we have $n_{i} \in\{-1,0,1\}$. The number $n_{+}$(respectively $n_{-}$) of those $i \in S(1, l)$ such that $n_{i}=1$ (respectively $n_{i}=-1$ ), is at most $f_{-1}$. Moreover $n_{+}+n_{-}=\varepsilon f_{-1} \leqslant$ $\varepsilon c=l$. Thus $n_{0}:=\min \left\{n_{+}, n_{-}\right\} \leqslant \frac{\varepsilon}{2} f_{-1}$.

We have $\mathbf{T}\left(\left\langle e_{1}, \ldots, e_{l}\right\rangle, \varphi\right) \leqslant n_{0}$ and there exist numbers $a_{i} \in S\left(0, n_{0}\right)$ such that $\left(\left\langle p^{a_{1}} e_{1}, \ldots\right.\right.$, $\left.\left.p^{a_{l}} e_{l}\right\rangle, \varphi\right)$ is a Dieudonné-Fontaine $p$-divisible object over $k$, cf. (3) and the proof of (2). Based on the maximal property of $E$, we can assume $\left\langle p^{a_{1}} e_{1}, \ldots, p^{a_{l}} e_{l}\right\rangle \subseteq\left\langle y_{1}, \ldots, y_{l}\right\rangle$ (i.e. $s_{i} \leqslant a_{i}$ for all $i \in S(1, l)$ ). If $l=c$, then the set $\left\{n_{+}, n_{-}\right\}$(and so also $n_{0}$ ) does not depend on the choice of the cycle $\left(y_{1}, \ldots, y_{l}\right)$. Thus we can choose $E$ such that $p^{n_{0}} \operatorname{Lie}(G) \subseteq E$. So any element of $G(W(k))$ congruent $\bmod p^{n_{0}+1}$ to $1_{M}$ belongs to $1_{M}+p E$. As $\operatorname{Lie}(T) \subseteq E, E$ is a $W(k)$ subalgebra of $\prod_{i=1}^{c} \operatorname{End}\left(M_{i}\right)$ and so also of $\operatorname{End}(M)$. Thus (cf. 3.3.2(b) applied with $G=G_{1}$ and with $\left.m_{1} \in S\left(0, n_{0}\right)\right)$ we have

$$
n \leqslant n_{0}+1=\min \left\{n_{+}, n_{-}\right\}+1 \leqslant \frac{\varepsilon}{2} f_{-1}+1 \leqslant c+1 .
$$

\subsection{Example 4}

Let $d \in \mathbf{N} \backslash\{1,2\}$. Let $D$ be a $p$-divisible group over $\operatorname{Spec}(k)$ of height $r=2 d$, dimension $d$, and slopes $\frac{1}{d}$ and $\frac{d-1}{d}$. Let $\left(M, \varphi_{0}\right)$ be the Dieudonné module of $D$; we have $r_{M}=r$. It is easy to see that we have a short exact sequence

$$
0 \rightarrow D_{2} \rightarrow D \rightarrow D_{1} \rightarrow 0
$$

of $p$-divisible groups over $\operatorname{Spec}(k)$, where the slopes of $D_{1}$ and $D_{2}$ are $\frac{d-1}{d}$ and $\frac{1}{d}$ (respectively). This short exact sequence is different from the classical slope filtration of $D$ (see [41, §3]) which is a short exact sequence $0 \rightarrow D_{1} \rightarrow D \rightarrow D_{2} \rightarrow 0$. As $D_{1}$ and $D_{2}$ are uniquely determined up to isomorphisms (see [5, Ch. IV, §8]), there exist a $W(k)$-basis $\left\{e_{1}, \ldots, e_{r}\right\}$ of $M$ and elements $x_{1}, \ldots, x_{d} \in\left\langle e_{1}, \ldots, e_{d}\right\rangle$ such that $\varphi_{0}$ takes the $r$-tuple $\left(e_{1}, \ldots, e_{r}\right)$ into $\left(e_{2}, p e_{3}, \ldots, p e_{d}, p e_{1}, e_{d+2}+x_{2}, \ldots, e_{r}+x_{d}, p e_{d+1}+p x_{1}\right)$. Let

$$
M_{1}:=\left\langle e_{1}, \ldots, e_{d}\right\rangle \quad \text { and } \quad M_{2}:=\left\langle e_{d+1}, \ldots, e_{r}\right\rangle .
$$

The pairs $\left(M_{1}, \varphi_{0}\right)$ and $\left(M / M_{1}, \varphi_{0}\right)$ are the Dieudonné modules of $D_{1}$ and $D_{2}$ (respectively). Let $\varphi$ be the $\sigma$-linear endomorphism of $M$ that takes $\left(e_{1}, \ldots, e_{r}\right)$ into $\left(e_{2}, p e_{3}, \ldots, p e_{d}, p e_{1}, e_{d+2}\right.$, $\left.\ldots, e_{r}, p e_{d+1}\right)$. Let $G:=\mathbf{G L}_{M}$.

Let $P_{1}$ and $P_{2}$ be the maximal parabolic subgroup schemes of $G$ that normalize $M_{1}$ and $M_{2}$ (respectively). Let $U_{1}$ and $U_{2}$ be the unipotent radicals of $P_{1}$ and $P_{2}$ (respectively). Let $u_{0} \in U_{1}(W(k))$ be the unique element such that $\varphi_{0}=u_{0} \varphi$. As $T$ we take the maximal torus of $G$ that normalizes $\left\langle e_{i}\right\rangle$ for all $i \in S(1, r)$. Let $L_{1}=\mathbf{G L}_{M_{1}} \times_{\operatorname{Spec}(W(k))} \mathbf{G L}_{M_{2}}$ be the unique Levi subgroup scheme of either $P_{1}$ or $P_{2}$ such that $T \leqslant L_{1}$, cf. [6, vol. III, Exp. XXVI, 1.12(ii)]. We have natural identifications $\operatorname{Lie}\left(L_{1}\right)=\operatorname{End}\left(M_{1}\right) \oplus \operatorname{End}\left(M_{2}\right), \operatorname{Lie}\left(U_{1}\right)=\operatorname{Hom}\left(M_{2}, M_{1}\right)$, and $\operatorname{Lie}\left(U_{2}\right)=\operatorname{Hom}\left(M_{1}, M_{2}\right)$. The triples $\left(M, \varphi_{0}, P_{1}\right)$ and $\left(M, \varphi_{0}, U_{1}\right)$ are latticed $F$-isocrystals with a group over $k$.

As $U_{1}$ is commutative we have $\left(\operatorname{Lie}\left(U_{1}\right), \varphi\right)=\left(\operatorname{Lie}\left(U_{1}\right), \varphi_{0}\right)$. We easily get that $\operatorname{Lie}\left(U_{1}\right)$ has a $W(k)$-basis $\left\{e_{i}^{(j)} \mid 1 \leqslant i, j \leqslant d\right\}$ such that $\varphi_{0}\left(e_{i}^{(j)}\right)=p^{n_{i}^{(j)}} e_{i+1}^{(j)}$, where each $d$-tuple 
$\left(n_{1}^{(j)}, n_{2}^{(j)}, \ldots, n_{d}^{(j)}\right)$ is either $(1,1, \ldots, 1,1,-1)$ or some $d$-tuple of the form $(1,1, \ldots, 1,0,1, \ldots$, $1,0)$. As $\mathbf{S}(1,1, \ldots, 1,-1)=1$ and $\mathbf{S}(1,1, \ldots, 1,0,1, \ldots, 1,0)=0$, from (2) applied to all pairs $\left(\left\langle e_{1}^{(j)}, \ldots, e_{d}^{(j)}\right\rangle, \varphi\right)$ we get $\mathbf{T}\left(\operatorname{Lie}\left(U_{1}\right), \varphi_{0}\right) \leqslant 1$. As $\operatorname{Lie}\left(U_{1}\right)$ is a $W(k)$-submodule of $\operatorname{End}(M)$ whose product with itself is the zero $W(k)$-submodule, the $i$-numbers of $\left(M, \varphi_{0}, U_{1}\right)$ and $\left(M, \varphi, U_{1}\right)$ are at most 1 (cf. 3.3.3). A similar argument shows that both $\mathbf{T}\left(\operatorname{Lie}\left(U_{2}\right), \varphi\right)$ and the $i$-number of $\left(M, \varphi, U_{2}\right)$ are at most 1 .

4.5.1. Proposition. - The p-divisible group $D$ is uniquely determined up to isomorphism by $D\left[p^{3}\right]$.

Proof. - Let $t \in \mathbf{N} \backslash\{1\}$. Let $g_{t} \in G$ be congruent $\bmod p^{t}$ to $1_{M}$. We will show that if $t \geqslant 3$, then there exists $g \in G(W(k))$ such that $g g_{t} \varphi_{0} g^{-1} \varphi_{0}^{-1} \in G(W(k))$ is congruent mod $p^{t+1}$ to $1_{M}$. The product morphism $U_{2} \times_{\operatorname{Spec}(W(k))} L_{1} \times_{\operatorname{Spec}(W(k))} U_{1} \rightarrow G$ is an open embedding around the identity section, cf. [6, vol. III, Exp. XXII, 4.1.2]. Thus we can write $g_{t}=u_{2} l_{1} u_{1}$, where the elements $u_{1} \in U_{1}(W(k)), l_{1} \in L_{1}(W(k))$, and $u_{2} \in U_{2}(W(k))$ are all congruent $\bmod p^{t}$ to $1_{M}$. As the $i$-number of $\left(M, \varphi_{0}, U_{1}\right)$ is at most 1 , to show the existence of $g$ we can replace $u_{0}$ by any other element of $U_{1}(W(k))$ that is congruent mod $p$ to $u_{0}$. Thus we can assume $u_{1}=1_{M}$.

For $i \in\{1,2\}$ let $E_{i}$ be the $W(k)$-span of the $\mathbf{Z}_{p}$-algebra of endomorphisms of $\left(M_{i}, \varphi\right)$. Let $E:=E_{1} \oplus E_{2}$. We have $p \operatorname{Lie}\left(L_{1}\right) \subseteq E$ (cf. 3.3.6 applied to both $D_{1}$ and $D_{2}$ ) and thus $l_{1} \in L_{1}(W(k)) \cap\left(1_{M}+p^{t-1} E\right)$. There exists $\tilde{l}_{1} \in L_{1}(W(k))$ congruent $\bmod p^{t-1}$ to $1_{M}$ and such that $\tilde{l}_{1} l_{1} \varphi \tilde{l}_{1}^{-1}=\varphi$, cf. 3.3.2(a) applied with $j=t-1$ to $\left(M, \varphi, L_{1}\right)$ and $E$. So, as $L_{1}$ normalizes both $U_{1}$ and $U_{2}$, by replacing $g_{t} \varphi_{0}$ with $\tilde{l}_{1} g_{t} \varphi_{0} \tilde{l}_{1}^{-1}, u_{2}$ with $\tilde{l}_{1} u_{2} \tilde{l}_{1}^{-1}$, and $u_{0}$ with the element $\tilde{l}_{1} l_{1} u_{0} l_{1}^{-1} \tilde{l}_{1}^{-1} \in U_{1}(W(k))$ congruent $\bmod p^{t-1}$ to $u_{0}$, we can assume $l_{1}$ is congruent $\bmod p^{t+1}$ to $1_{M}$. This implies that $g_{t}$ and $u_{2}$ are congruent $\bmod p^{t+1}$.

As $\mathbf{T}\left(\operatorname{Lie}\left(U_{2}\right), \varphi\right) \leqslant 1$, from 3.3.2(a) applied in a way similar to the one of the previous paragraph we deduce the existence of $\tilde{u}_{2} \in U_{2}(W(k))$ congruent $\bmod p^{t-1}$ to $1_{M}$ and such that $\tilde{u}_{2} u_{2} \varphi \tilde{u}_{2}^{-1}=\varphi$. As $g_{t}$ and $u_{2}$ are congruent $\bmod p^{t+1}$, the element

$$
g_{t}^{\prime}:=\tilde{u}_{2} g_{t} \varphi_{0} \tilde{u}_{2}^{-1} \varphi_{0}^{-1}=\tilde{u}_{2} g_{t} u_{0} \varphi \tilde{u}_{2}^{-1} \varphi^{-1} u_{0}^{-1}=\tilde{u}_{2} g_{t} u_{0} u_{2}^{-1} \tilde{u}_{2}^{-1} u_{0}^{-1} \in G(W(k))
$$

is congruent $\bmod p^{t+1}$ to the commutator of $\tilde{u}_{2} u_{2}$ and $u_{0}$. A simple matrix computation of this commutator shows that for $t \geqslant 3$ we can write $g_{t}^{\prime}=u_{2}^{\prime} l_{2}^{\prime} u_{1}^{\prime}$, where $u_{1}^{\prime} \in U_{1}(W(k))$ and $l_{1}^{\prime} \in L_{1}(W(k))$ are congruent $\bmod p^{t-1}$ to $1_{M}$ and where $u_{2}^{\prime} \in U_{2}(W(k))$ is congruent $\bmod p^{2 t-2}$ and so also mod $p^{t+1}$ to $1_{M}$ (here is the only place where we need $t \neq 2$ ).

Repeating twice the above part that allowed us to assume that $u_{1}$ and $l_{1}$ are congruent $\bmod p^{t+1}$ to $1_{M}$, we get that for $t \geqslant 3$ we can assume $g_{t}^{\prime}$ is congruent $\bmod p^{t+1}$ to $u_{2}^{\prime}$ and thus also to $1_{M}$. This ends the argument for the existence of $g$.

Thus for $t \geqslant 3$ we have $t+1 \neq n$; so $n \leqslant 3$. So the proposition follows from 3.2.3.

\subsubsection{Notations for $d=3$}

Let $d$ be 3. For $\alpha \in W(k)$ let $\varphi_{\alpha}$ be the $\sigma$-linear endomorphism of $M$ that takes $\left(e_{1}, \ldots, e_{6}\right)$ into $\left(e_{2}, p e_{3}, p e_{1}, e_{5}, e_{6}+\alpha e_{1}, p e_{4}\right)$. The slopes of $\left(M, \varphi_{\alpha}\right)$ are $\frac{1}{3}$ and $\frac{2}{3}$. For $(i, j) \in\{1,2,3\} \times$ $\{4,5,6\}$ let $U_{i j}$ be the unique $\mathbf{G}_{a}$ subgroup scheme of $U_{1}$ that is normalized by $T$, that fixes $e_{j^{\prime}}$ for $j^{\prime} \in\{4,5,6\} \backslash\{j\}$, and that takes $e_{j}$ into $e_{j}+\left\langle e_{i}\right\rangle$. Let $n_{\alpha} \in U_{16}(W(k))$ be the unique element such that $\varphi_{\alpha}=n_{\alpha} \varphi$.

4.5.3. Proposition. - Suppose $d=3$. Let $\alpha_{1}, \alpha_{2} \in W(k) \backslash\{0\}$ be such that the $\mathbf{F}_{p^{3}}$-vector subspace of $k$ generated by $\alpha_{1} \bmod p$ is different from the $\mathbf{F}_{p^{3}}$-vector subspace of $k$ generated by $\alpha_{2} \bmod p$. Then $\left(M, \varphi_{\alpha_{1}}\right)$ and $\left(M, \varphi_{\alpha_{2}}\right)$ are not isomorphic. 
Proof. - We show that the assumption that there exists $g_{1} \in G(W(k))$ which is an isomorphism between $\left(M, \varphi_{\alpha_{1}}\right)$ and $\left(M, \varphi_{\alpha_{2}}\right)$ leads to a contradiction. As $\operatorname{Lie}\left(P_{1}\right)\left[\frac{1}{p}\right]$ is the maximal direct summand of $\operatorname{End}\left(M\left[\frac{1}{p}\right]\right)$ normalized by $\varphi_{\alpha}$ and such that all slopes of $\left(\operatorname{Lie}\left(P_{1}\right)\left[\frac{1}{p}\right], \varphi_{\alpha}\right)$ are non-negative, the element $g_{1} \in G(W(k))$ normalizes $\operatorname{Lie}\left(P_{1}\right)$ and so also $P_{1}$. Thus $g_{1} \in P_{1}(W(k))$. We write $g_{1}=u_{1} l_{1}$, where $u_{1} \in U_{1}(W(k))$ and $l_{1} \in L_{1}(W(k))$. We have $u_{1} l_{1} n_{\alpha_{1}} l_{1}^{-1} l_{1} \varphi=n_{\alpha_{2}} \varphi u_{1} \varphi^{-1} \varphi l_{1}$. As $u_{1} l_{1} n_{\alpha_{1}} l_{1}^{-1}$ and $n_{\alpha_{2}} \varphi u_{1} \varphi^{-1}$ belong to $U_{1}(W(k))$, the actions of $l_{1} \varphi$ and $\varphi l_{1}$ on both $M_{1}$ and $M / M_{1}$ are equal. So as $l_{1} \varphi$ and $\varphi l_{1}$ both normalize the direct supplement $M_{2}\left[\frac{1}{p}\right]$ of $M_{1}\left[\frac{1}{p}\right]$ in $M\left[\frac{1}{p}\right]$, we get $l_{1} \varphi=\varphi l_{1}$; so we also have $u_{1} l_{1} n_{\alpha_{1}} l_{1}^{-1}=$ $n_{\alpha_{2}} \varphi u_{1} \varphi^{-1}$. As $l_{1} \varphi=\varphi l_{1}$, a simple computation shows that $l_{1}$ takes $e_{1}$ (respectively $e_{6}$ ) into $a_{1} e_{1}+b_{1} e_{2}+c_{1} e_{3}$ (respectively $p a_{6} e_{4}+p b_{6} e_{5}+c_{6} e_{6}$ ), where $a_{1}, b_{1}, c_{1} \in W\left(\mathbf{F}_{p^{3}}\right)$ (respectively $a_{6}, b_{6} \in W\left(\mathbf{F}_{p^{3}}\right)$ and $\left.c_{6} \in \mathbf{G}_{m}\left(W\left(\mathbf{F}_{p^{3}}\right)\right)\right)$. As $U_{1}=\prod_{i=1}^{3} \prod_{j=4}^{6} U_{i j}$, there exist unique elements $u_{i j} \in U_{i j}(W(k))$ such that we have $u_{1}=\prod_{i=1}^{3} \prod_{j=4}^{6} u_{i j}$. We call $u_{i j}$ as the component of $u_{1}$ in $U_{i j}(W(k))$. Both $g_{1} \bmod p$ and $l_{1} \bmod p$ normalize the kernel of $\varphi_{\alpha}$ 's $\bmod p$, i.e. they normalize $\left\langle e_{2}, e_{3}, e_{6}\right\rangle \bmod p$. So $u_{1} \bmod p$ also normalizes $\left\langle e_{2}, e_{3}, e_{6}\right\rangle \bmod p$. If $(i, j) \neq(1,6)$, then $u_{i j} \bmod p$ normalizes $\left\langle e_{2}, e_{3}, e_{6}\right\rangle \bmod p$. Thus $u_{16} \bmod p$ normalizes $\left\langle e_{2}, e_{3}, e_{6}\right\rangle \bmod p$ and therefore it is $1_{M / p M}$. As $U_{35}$ fixes both $\left\langle e_{2}, e_{3}, e_{6}\right\rangle$ and $M /\left\langle e_{2}, e_{3}, e_{6}\right\rangle, \varphi\left(u_{35}\right) \bmod p$ is $1_{M / p M}$. Thus the component of $n_{\alpha_{2}} \varphi u_{1} \varphi^{-1}$ in $U_{16}(W(k))$ is congruent $\bmod p$ to $n_{\alpha_{2}}$. The component of $l_{1} n_{\alpha_{1}} l_{1}^{-1}$ in $U_{16}(W(k))$ is $n_{\alpha_{3}}$, where $\alpha_{3}:=a_{1} \alpha_{1} c_{6}^{-1} \in W(k)$. So $\alpha_{3} \bmod p$ belongs to the $\mathbf{F}_{p^{3}}$-vector subspace of $k$ generated by $\alpha_{1} \bmod p$. The component of $u_{1} l_{1} n_{\alpha_{1}} l_{1}^{-1}$ in $U_{16}(W(k))$ is congruent $\bmod p$ to $n_{\alpha_{3}}$. As $u_{1} l_{1} n_{\alpha_{1}} l_{1}^{-1}=n_{\alpha_{2}} \varphi u_{1} \varphi^{-1}$, we get that $n_{\alpha_{2}}$ and $n_{\alpha_{3}}$ are congruent $\bmod p$. So $\alpha_{2} \bmod p$ belongs to the $\mathbf{F}_{p^{3}}$-vector subspace of $k$ generated by $\alpha_{1} \bmod p$. This contradicts our hypothesis. Thus $g_{1}$ does not exist.

4.5.4. Remark. - The set of isomorphism classes of $p$-divisible groups over $\operatorname{Spec}(k)$ of height 6 and dimension 3 has the same cardinality as $k$, cf. 4.5.3, 1.3, and the classical Dieudonné theory. But the set of isomorphism classes of $p$-torsion subgroup schemes of such $p$-divisible groups over $\operatorname{Spec}(k)$ is finite (see [26]; to be compared with [34, §1]). Based on this, 4.5.1, and 3.2.3, we get that the set of those elements $\alpha \in \mathbf{G}_{m}(W(k))$ for which the $i$-number of $\left(M, \varphi_{\alpha}\right)$ is either 2 or 3 , has the same cardinality as $k$. Let $\lambda_{M}$ be the perfect alternating form on $M$ defined by the rule: if $1 \leqslant i<j \leqslant 6$, then $\lambda_{M}\left(e_{i}, e_{j}\right) \in\{0,1\}$ and we have $\lambda_{M}\left(e_{i}, e_{j}\right)=1$ if and only if $(i, j) \in\{(1,6),(3,5),(2,4)\}$. The form $\lambda_{M}$ is a principal quasi-polarization of any $\left(M, \varphi_{\alpha}\right)$.

From 4.1.1 and 4.5.1 we easily get that $T(6,3) \leqslant 9$.

\section{Four direct applications}

In this section we continue to assume that $k=\bar{k}$.

\subsection{The homomorphism form of 1.2}

Let $\left(M_{1}, \varphi_{1}\right)$ and $\left(M_{2}, \varphi_{2}\right)$ be two $F$-crystals over $k$. Let $(M, \varphi):=\left(M_{1}, \varphi_{1}\right) \oplus\left(M_{2}, \varphi_{2}\right)$. For $i \in\{1,2\}$, let $h_{i}$ be the $h$-number of $\left(M_{i}, \varphi_{i}\right)$. Let $h_{12}:=\max \left\{h_{1}, h_{2}\right\}$, let $m_{12}:=$ $\mathbf{T}(\operatorname{End}(M), \varphi)$, let $v_{12}:=m_{12}+h_{12}$, let $\varepsilon_{p} \in\{1,2\}$ be as before 3.1, and let $n_{12}:=m_{12}+\varepsilon_{p}$. The $h$-number of $(M, \varphi)$ is $h_{12}$.

5.1.1. TheOREM. - We endow $(\mathbf{N} \cup\{0\})^{2}$ with the lexicographic order. We have:

(a) For all $t \in \mathbf{N} \cup\{0\}$, the images defined via restrictions of the two groups $\operatorname{Hom}\left(\left(M_{1}, \varphi_{1}\right),\left(M_{2}, \varphi_{2}\right)\right)$ and $\operatorname{Hom}\left(\left(M_{1} / p^{n_{12}+v_{12}+t} M_{1}, \varphi_{1}\right),\left(M_{2} / p^{n_{12}+v_{12}+t} M_{2}, \varphi_{2}\right)\right)$ in the group $\operatorname{Hom}\left(\left(M_{1} / p^{n_{12}+t} M_{1}, \varphi_{1}\right),\left(M_{2} / p^{n_{12}+t} M_{2}, \varphi_{2}\right)\right)$, coincide. 
(b) We fix a quadruple $\left(r_{1}, r_{2}, c_{1}, c_{2}\right) \in(\mathbf{N} \cup\{0\})^{4}$. There exists a smallest pair

$$
(v, n):=\left(v\left(r_{1}, r_{2}, c_{1}, c_{2}\right), n\left(r_{1}, r_{2}, c_{1}, c_{2}\right)\right) \in(\mathbf{N} \cup\{0\})^{2}
$$

with the properties that $n \in S\left(0, v+\varepsilon_{p}-\max \left\{c_{1}, c_{2}\right\}\right)$ and that for any $t \in \mathbf{N} \cup\{0\}$ and for every two F-crystals $\left(M_{1}, \varphi_{1}\right)$ and $\left(M_{2}, \varphi_{2}\right)$ over $k$ which satisfy $\left(r_{M_{1}}, r_{M_{2}}, h_{1}, h_{2}\right)=$ $\left(r_{1}, r_{2}, c_{1}, c_{2}\right)$, the images defined via restrictions of the two groups $\operatorname{Hom}\left(\left(M_{1}, \varphi_{1}\right),\left(M_{2}, \varphi_{2}\right)\right)$ and $\operatorname{Hom}\left(\left(M_{1} / p^{n+v+t} M_{1}, \varphi_{1}\right),\left(M_{2} / p^{n+v+t} M_{2}, \varphi_{2}\right)\right)$ in the group $\operatorname{Hom}\left(\left(M_{1} / p^{n+t} M_{1}, \varphi_{1}\right),\left(M_{2} / p^{n+t} M_{2}, \varphi_{2}\right)\right)$, coincide. The number $v$ (and so also $n$ ) has upper bounds that depend only on $r_{1}, r_{2}$, and $\max \left\{c_{1}, c_{2}\right\}$.

(c) Let $r_{1}, r_{2} \in \mathbf{N}$. There exists a smallest pair

$$
(v, n):=\left(v\left(r_{1}, r_{2}\right), n\left(r_{1}, r_{2}\right)\right) \in(\mathbf{N} \cup\{0\})^{2}
$$

with the properties that $n \in S\left(0, v+\varepsilon_{p}-1\right)$ and that for any $t \in \mathbf{N} \cup\{0\}$ and for every two p-divisible groups $D_{1}$ and $D_{2}$ over $\operatorname{Spec}(k)$ of heights $r_{1}$ and $r_{2}$, a homomorphism $D_{1}\left[p^{n+t}\right] \rightarrow D_{2}\left[p^{n+t}\right]$ lifts to a homomorphism $D_{1} \rightarrow D_{2}$ if and only if it lifts to a homomorphism $D_{1}\left[p^{n+v+t}\right] \rightarrow D_{2}\left[p^{n+v+t}\right]$. The number $v$ (and so also $n$ ) has upper bounds that depend only on $r_{1}$ and $r_{2}$.

Proof. - Let $\quad e_{12} \in \operatorname{Hom}\left(\left(M_{1} / p^{n_{12}+v_{12}+t} M_{1}, \varphi_{1}\right),\left(M_{2} / p^{n_{12}+v_{12}+t} M_{2}, \varphi_{2}\right)\right)$. Let $g \in \operatorname{Aut}\left(M / p^{n_{12}+v_{12}+t} M, \varphi\right)$ be such that it takes $x_{2} \in M_{2} / p^{n_{12}+v_{12}+t} M_{2}$ into $x_{2}$ and it takes $x_{1} \in M_{1} / p^{n_{12}+v_{12}+t} M_{1}$ into $x_{1}+e_{12}\left(x_{1}\right)$. Let $\tilde{g} \in \operatorname{Aut}(M, \varphi)$ be such that it lifts the reduction $\bmod p^{n_{12}+t}$ of $g$, cf. 3.2.8(b). Let $\tilde{e}_{12}: M_{1} \rightarrow M_{2}$ be the $W(k)$-linear map such that we have $\tilde{g}\left(x_{1}\right)-\tilde{e}_{12}\left(x_{1}\right) \in M_{1}$ for all $x_{1} \in M_{1}$. We have $\tilde{e}_{12} \in \operatorname{Hom}\left(\left(M_{1}, \varphi_{1}\right),\left(M_{2}, \varphi_{2}\right)\right)$ and moreover $e_{12}$ and $\tilde{e}_{12}$ have the same image in $\operatorname{Hom}\left(\left(M_{1} / p^{n_{12}+t} M_{1}, \varphi_{1}\right),\left(M_{2} / p^{n_{12}+t} M_{2}, \varphi_{2}\right)\right)$. This proves (a).

We know that $m_{12}$ has an upper bound $b_{12} \in \mathbf{N}$ which is effectively computable in terms of $2 h_{12}=2 \max \left\{c_{1}, c_{2}\right\}$ and $r_{M}^{2}=\left(r_{M_{1}}+r_{M_{2}}\right)^{2}=\left(r_{1}+r_{2}\right)^{2}$ (cf. 2.4.1 and end of 2.2.1(e)) and that we have $v \leqslant m_{12}+h_{12}$ (cf. (a)). From this (b) follows.

To prove (c), for $i \in\{1,2\}$ we take $\left(M_{i}, \varphi_{i}\right)$ to be the Dieudonné module of $D_{i}$. We have $h_{12} \in\{0,1\}$. If $h_{12}=0$, then $h_{1}=h_{2}=0$ and so both $D_{1}$ and $D_{2}$ are étale $p$-divisible groups; in such a case any homomorphism $D_{1}\left[p^{n+t}\right] \rightarrow D_{2}\left[p^{n+t}\right]$ lifts to a homomorphism $D_{1} \rightarrow D_{2}$. Thus we can assume that $h_{12}=1$. Based on 3.2.3, the proofs of (a) and (b) can be adapted to the context of $p$-divisible groups; thus (c) follows from the particular case of (b) when $\max \left\{c_{1}, c_{2}\right\}$ is $h_{12}=1$.

5.1.2. Remark. - If $v_{1}, n_{1} \in \mathbf{N}$ are such that $v_{1} \geqslant v$ and $n_{1} \geqslant n$, then the homomorphisms parts of 5.1.1(b) and (c) continue to hold if we replace $(v, n)$ with $\left(v_{1}, n_{1}\right)$.

\subsection{Transcendental degrees of definition}

For simplicity, in this section we work in a context without principal bilinear quasipolarizations (but we emphasize that all of 5.2 can be adapted to the context of 2.2.1(c)). Let $\left(M, \varphi, G,\left(t_{\alpha}\right)_{\alpha \in \mathcal{J}}\right)$ be a latticed $F$-isocrystal with a group and an emphasized family of tensors over $k$ such that the $W$-condition holds for $(M, \varphi, G)$. Let $\left(F^{i}(M)\right)_{i \in S(a, b)}$ be a lift of $(M, \varphi, G)$.

Let $\mu:=\mu_{\text {can }}: \mathbf{G}_{m} \rightarrow G$ be the inverse of the canonical split cocharacter of $\left(M,\left(F^{i}(M)\right)_{i \in S(a, b)}, \varphi\right)$ (see 2.5). Let $\left(M_{\mathbf{Z}_{p}}, G_{\mathbf{Z}_{p}}\right)$ be the $\mathbf{Z}_{p}$ structure of $(M, G)$ that is defined as in 2.5 by the $\sigma$-linear automorphism $\sigma_{0}:=\varphi \mu(p): M \stackrel{\sim}{\longrightarrow} M$; thus $\sigma_{0}$ fixes $M_{\mathbf{Z}_{p}}$ and 
normalizes $G(W(k))$ and moreover we have $t_{\alpha} \in \mathcal{T}\left(M_{\mathbf{Z}_{p}}\right)$ for all $\alpha \in \mathcal{J}$. Let $n \in \mathbf{N} \cup\{0\}$ be the $i$-number of $(M, \varphi, G)$.

5.2.1. FACT. - Let $k_{1}$ be the smallest subfield of $k$ with the property that $\mu_{W_{n}(k)}$ is the pullback of a cocharacter $\mu_{1 W_{n}\left(k_{1}\right)}: \mathbf{G}_{m} \rightarrow G_{\mathbf{Z}_{p}} \times_{\operatorname{Spec}\left(\mathbf{Z}_{p}\right)} \operatorname{Spec}\left(W_{n}\left(k_{1}\right)\right)$. Then the field $k_{1}$ is finitely generated and its transcendental degree $t\left(k_{1}\right)$ is at most $n r_{M}^{2}$. Thus if $t\left(k_{1}\right)=0$, then $k_{1}$ is a finite field.

Proof. - Let $\mathcal{B}$ be a $W(k)$-basis of $M$ such that $\mu$ normalizes the $W(k)$-spans of elements of $\mathcal{B}$. Let $\mathcal{B}_{1}$ be a $\mathbf{Z}_{p}$-basis of $M_{\mathbf{Z}_{p}}$; we also view it as a $W(k)$-basis of $M$. Let $B \in M_{r_{M} \times r_{M}}(W(k))$ be the change of coordinates matrix that changes $\mathcal{B}_{1}$-coordinates into $\mathcal{B}$-coordinates. Let $R_{1}$ be the $\mathbf{F}_{p}$-subalgebra of $k$ generated by the coordinates of the Witt vectors of length $n$ with coefficients in $k$ that are entries of $B \bmod p^{n}$. Obviously $k_{1}$ is a subfield of the field of fractions of $R_{1}$. As $R_{1}$ is generated by $n r_{M}^{2}$ elements, $k_{1}$ is finitely generated and we have $\operatorname{dim}\left(\operatorname{Spec}\left(R_{1}\right)\right) \leqslant n r_{M}^{2}$. Thus $t\left(k_{1}\right) \leqslant n r_{M}^{2}$.

Until 5.3 we take $G$ to be smooth over $\operatorname{Spec}(W(k))$. Thus for any $l \in \mathbf{N}$ there exists a cocharacter $\mu_{1, n+l}$ of $G_{\mathbf{Z}_{p}} \times_{\operatorname{Spec}\left(\mathbf{Z}_{p}\right)} \operatorname{Spec}\left(W_{n+l}\left(k_{1}^{\text {perf }}\right)\right)$ that lifts the pullback $\mu_{1 W_{n}\left(k_{1}^{\text {perf }}\right)}$ to $\operatorname{Spec}\left(W_{n}\left(k_{1}^{\text {perf }}\right)\right)$ of $\mu_{1 W_{n}\left(k_{1}\right)}$, cf. [6, vol. II, Exp. IX, 3.6]. We can assume that $\mu_{1, n+l+1}$ lifts $\mu_{1, n+l}$, cf. loc. cit. From [6, vol. II, Exp. IX, 7.1] we get that there exists a unique cocharacter $\mu_{1}: \mathbf{G}_{m} \rightarrow G_{\mathbf{Z}_{p}} \times \operatorname{Spec}\left(\mathbf{Z}_{p}\right) \operatorname{Spec}\left(W\left(k_{1}^{\text {perf }}\right)\right)$ that lifts all $\mu_{1, n+l}$ 's.

The cocharacter $\mu_{1 W(k)}: \mathbf{G}_{m} \rightarrow G$ is of the form $g_{n} \mu g_{n}^{-1}$ for some $g_{n} \in G(W(k))$ congruent $\bmod p^{n}$ to $1_{M}$, cf. [6, vol. II, Exp. IX, 3.6] and the fact that we have $G(W(k))=$ proj $\lim _{l \in \mathbf{N}} G\left(W_{n+l}(k)\right)$. Let $\tilde{g}_{n}:=g_{n}^{-1} \sigma_{0} g_{n} \sigma_{0}^{-1} \in G(W(k))$; it is congruent $\bmod p^{n}$ to $1_{M}$. The element $g_{n}^{-1} \in G(W(k))$ defines an isomorphism between $\left(M, \sigma_{0} \mu_{1 W(k)}\left(\frac{1}{p}\right), G,\left(t_{\alpha}\right)_{\alpha \in \mathcal{J}}\right)$ and $\left(M, \tilde{g}_{n} \varphi, G,\left(t_{\alpha}\right)_{\alpha \in \mathcal{J}}\right)$. Moreover $\left(M, \varphi, G,\left(t_{\alpha}\right)_{\alpha \in \mathcal{J}}\right)$ and $\left(M, \tilde{g}_{n} \varphi, G,\left(t_{\alpha}\right)_{\alpha \in \mathcal{J}}\right)$ are isomorphic under an isomorphism defined by an element of $G(W(k))$, cf. the very definition of $n$. We conclude that:

$(*)$ the quadruple $\left(M, \varphi, G,\left(t_{\alpha}\right)_{\alpha \in \mathcal{J}}\right)$ is isomorphic to $\left(M, \sigma_{0} \mu_{1 W(k)}\left(\frac{1}{p}\right), G,\left(t_{\alpha}\right)_{\alpha \in \mathcal{J}}\right)$; thus $\left(M, \varphi, G,\left(t_{\alpha}\right)_{\alpha \in \mathcal{J}}\right)$ is definable over $k_{1}^{\text {perf }}$ and moreover its isomorphism class is uniquely determined by the triple $\left(M_{\mathbf{Z}_{p}},\left(t_{\alpha}\right)_{\alpha \in \mathcal{J}}, \mu_{1 W_{n}\left(k_{1}\right)}\right)$.

This motivates the following definitions.

\subsubsection{DEFINITIONS. -}

(a) We say $k_{1}$ (respectively $t\left(k_{1}\right)$ ) is the field (respectively the transcendental degree) of definition of $(M, \varphi, G)$ or of $\left(M, \varphi, G,\left(t_{\alpha}\right)_{\alpha \in \mathcal{J}}\right)$ with respect to the lift $\left(F^{i}(M)\right)_{i \in S(a, b)}$ of $(M, \varphi, G)$. By the transcendental degree of definition $t d \in \mathbf{N} \cup\{0\}$ of $(M, \varphi, G)$ or of $\left(M, \varphi, G,\left(t_{\alpha}\right)_{\alpha \in \mathcal{J}}\right)$ we mean the smallest number we get by considering transcendental degrees of definition of $(M, \varphi, G)$ with respect to (arbitrary) lifts of it.

(b) If $t d=0$, then by the field of definition of $(M, \varphi, G)$ or of $\left(M, \varphi, G,\left(t_{\alpha}\right)_{\alpha \in \mathcal{J}}\right)$ we mean the finite field that has the smallest number of elements and that is the field of definition of $(M, \varphi, G)$ with respect to some lift of it.

If $t d=0$ we do not stop to study when the field of definition of $(M, \varphi, G)$ is contained in all fields of definition of $(M, \varphi, G)$ with respect to (arbitrary) lifts of $(M, \varphi, G)$.

5.2.3. THEOREM (Atlas Principle). - We recall that $k=\bar{k}$, that $G$ is smooth over $\operatorname{Spec}(W(k))$, and that the $W$-condition holds for $(M, \varphi, G)$. Let $q \in \mathbf{N}$. Let $\mathcal{I}(q) \in \mathbf{N} \cup\{0, \infty\}$ be the number of isomorphism classes of latticed $F$-isocrystals with a group and an emphasized family of tensors over $k$ that have the form $\left(M, g \varphi, G,\left(t_{\alpha}\right)_{\alpha \in \mathcal{J}}\right)$ for some $g \in G(W(k))$, that have tran- 
scendental degrees of definition 0 , and that have $\mathbf{F}_{p^{q}}$ as their fields of definition. Then we have $\mathcal{I}(q) \in \mathbf{N} \cup\{0\}$.

Proof. - We check that the number $N_{\text {tor }} \in \mathbf{N} \cup\{\infty\}$ of isomorphism classes of pairs of the form $\left(M_{\mathbf{Z}_{p}},\left(t_{\alpha}\right)_{\alpha \in \mathcal{J}}\right)$ that are obtained by replacing $\varphi$ with some $g \varphi$ and by considering some lift of $(M, g \varphi, G)$, is finite. The "difference" between two such pairs is measured by a torsor $\Theta$ of $G_{\mathbf{Z}_{p}}$ in the flat topology of $\operatorname{Spec}\left(\mathbf{Z}_{p}\right)$. So $\Theta$ is smooth over $\operatorname{Spec}\left(\mathbf{Z}_{p}\right)$. Thus $\Theta$ is a trivial torsor if and only if $\Theta_{\mathbf{F}_{p}}$ is a trivial torsor. As the set $H^{1}\left(\mathbf{F}_{p}, G_{\mathbf{F}_{p}}\right)$ is finite (cf. [38, Ch. III, $\S 4$, 4.2 and 4.3]), the number of isomorphism classes of torsors of $G_{\mathbf{F}_{p}}$ is finite. From the last two sentences we get that $N_{\text {tor }} \in \mathbf{N}$.

Let $m \in \mathbf{N}$. We check that the number $N\left(\mu, m, q, G_{\mathbf{Z}_{p}}\right) \in \mathbf{N} \cup\{0, \infty\}$ of cocharacters of $G_{\mathbf{Z}_{p}} \times_{\operatorname{Spec}\left(\mathbf{Z}_{p}\right)} \operatorname{Spec}\left(W_{m}\left(\mathbf{F}_{p^{q}}\right)\right)$ that over $\operatorname{Spec}\left(W_{m}(k)\right)$ are $G\left(W_{m}(k)\right)$-conjugate to $\mu_{W_{m}(k)}$, is also finite. Based on the infinitesimal liftings of [6, vol. II, Exp. IX, 3.6] and on the fact that the group $G_{\mathbf{Z}_{p}}\left(W_{m}\left(\mathbf{F}_{p^{q}}\right)\right)$ is finite, it is enough to prove that the number $N\left(\mu, 1, q, G_{\mathbf{Z}_{p}}\right)$ is finite. It suffices to prove that $N\left(\mu, 1, q, \mathbf{G L}_{M_{\mathbf{z}_{p}}}\right) \in \mathbf{N}$. The number of maximal split tori of $\mathbf{G L}_{M_{\mathbf{z}_{p}} \otimes \mathbf{z}_{p} \mathbf{F}_{p} q}$ is finite and each such torus has precisely $(b-a+1)^{r_{M}}$ cocharacters that act on $M_{\mathbf{z}_{p}} \otimes \mathbf{z}_{p} \mathbf{F}_{p^{q}}$ via those $-i$-th powers of the identity character of $\mathbf{G}_{m}$ that satisfy $i \in S(a, b)$. Thus $N\left(\mu, 1, q, \mathbf{G L}_{M_{\mathbf{z}_{p}}}\right) \in \mathbf{N}$.

Let $n_{\mathrm{fam}}$ be as in 3.1.5; we have $n_{\mathrm{fam}} \geqslant n$. Based on $5.2(*)$, we get that $\mathcal{I}(q)$ is bounded from above by a sum of $N_{\text {tor }}$ numbers of the form $N\left(\mu, n_{\mathrm{fam}}, q, G_{\mathbf{Z}_{p}}\right)$ and in particular that $\mathcal{I}(q) \leqslant N_{\text {tor }} N\left(\mu, n_{\text {fam }}, q, \mathbf{G L}_{M_{\mathbf{z}_{p}}}\right)$. Thus $\mathcal{I}(q) \in \mathbf{N} \cup\{0\}$.

\subsection{Groupoids and stratifications}

Main Theorem A has many reformulations in terms of (stacks of) groupoids. Not to increase the length of the paper, we postpone to future work the introduction of Shimura (stacks of) groupoids that parametrize isomorphism classes of Shimura $p$-divisible objects we defined in the beginning of Section 4. Presently, this Shimura context is the most general context to which we can extend the classical deformation theories of $p$-divisible groups (see [30, Chs. 4 and 5], [21, Chs. 3 and 4], [11, 7.1], [8, Main Thm. of Introd.], [9, Main Thm. 1], and [12, §7]). Here, as an anticipation of the numerous possibilities offered by 1.2 , we work only with (principally quasi-polarized) $p$-divisible groups. However, we point out that based on 3.2.2, by using $[40,5.4]$ as a substitute for the deformation theory of $[21,4.8]$, the below proof of 5.3.1 can be adapted to contexts that involve arbitrary Shimura varieties of Hodge type and thus involve (principally quasi-polarized) Dieudonné modules equipped with smooth groups as in 3.2 (like the context of $[40, \S 5])$.

Let $S$ be a reduced $\operatorname{Spec}\left(\mathbf{F}_{p}\right)$-scheme. Let $\mathcal{D}$ be a $p$-divisible group over $S$ of height $r$ and relative dimension $d$. For $i \in\{1,2\}$ let $\mathcal{D}_{i}$ be the pullback of $\mathcal{D}$ to $S_{12}:=S \times_{\operatorname{Spec}\left(\mathbf{F}_{p}\right)} S$ via the $i$-th projection $p_{i}: S_{12} \rightarrow S$. For $l \in \mathbf{N}$, let $I_{l}$ be the affine $S_{12}$-scheme that parametrizes isomorphisms between $\mathcal{D}_{1}\left[p^{l}\right]$ and $\mathcal{D}_{2}\left[p^{l}\right]$; it is of finite presentation. The morphism $i_{l}: I_{l} \rightarrow S_{12}$ of $S$-schemes is a $\operatorname{Spec}\left(\mathbf{F}_{p}\right)$-groupoid that acts on $S$ in the sense of [6, vol. I, Exp. V] and [31, Appendix A].

\subsubsection{BASIC THEOREM. -}

(a) There exists a number $l \in \mathbf{N}$ effectively bounded from above only in terms of $r$ and such that for any algebraically closed field $K$ of characteristic $p$, the pullbacks of $\mathcal{D}$ through two $K$-valued points $y_{1}$ and $y_{2}$ of $S$ are isomorphic if and only if the $K$-valued point of $S_{12}$ defined by the pair $\left(y_{1}, y_{2}\right)$ factors through $i_{l}: I_{l} \rightarrow S_{12}$.

(b) Suppose $S$ is smooth over $\operatorname{Spec}\left(\mathbf{F}_{p}\right)$ of dimension $d(r-d)$ and $\mathcal{D}$ is a versal deformation at each maximal point of $S$. Then there exists a stratification $\mathcal{S}(\mathcal{D})$ of $S$ in reduced, 
locally closed subschemes such that two points $y_{1}$ and $y_{2}$ as in (a) factor through the same stratum if and only if $y_{1}^{*}(\mathcal{D})$ is isomorphic to $y_{2}^{*}(\mathcal{D})$. The strata of $\mathcal{S}(\mathcal{D})$ are regular and equidimensional.

(c) The stratification $\mathcal{S}(\mathcal{D})$ of (b) satisfies the purity property.

(d) Let $q \in \mathbf{N}$ and let $K$ be as in (a). Then for any stratum $S_{0}$ of the stratification $\mathcal{S}(\mathcal{D})$ of (b) that is a subscheme of $S_{K}$, there exists a regular scheme $S_{0}[q]$ that is finite, flat over $S_{0}$ and such that the pullback of $\mathcal{D}\left[p^{q}\right]$ to $S_{0}[q]$ is constant, i.e. is the pullback to $S_{0}[q]$ of a truncated Barsotti-Tate group of level q over $\operatorname{Spec}(K)$.

Proof. - Part (a) follows from 1.3: as $l$ we can take any integer greater that $T(r, d)$. For the rest of the proof we will assume that $S$ is smooth over $\operatorname{Spec}\left(\mathbf{F}_{p}\right)$ of dimension $d(r-d)$ and that $\mathcal{D}$ is a versal deformation at each maximal point of $S$. We first construct the strata of $\mathcal{S}(\mathcal{D})$ that are subschemes of $S_{K}$. Let $y_{1} \in S(K)=S_{K}(K)$. Let

$$
S\left(y_{1}\right)^{\mathrm{top}}:=\left(p_{2} \circ i_{l}\right)_{K}\left(\left(p_{1} \circ i_{l}\right)_{K}^{-1}\left(y_{1}\right)\right) \subseteq S_{K}^{\mathrm{top}} .
$$

As $I_{l}$ is a $\operatorname{Spec}\left(\mathbf{F}_{p}\right)$-scheme of finite type, $S\left(y_{1}\right)^{\text {top }}$ is a constructible subset of $S_{K}^{\text {top }}$ (cf. [18, Ch. IV, (1.8.4) and (1.8.5)]). Let $\bar{S}\left(y_{1}\right)$ be the Zariski closure of $S\left(y_{1}\right)^{\text {top }}$ in $S_{K}$; it is a reduced, closed subscheme of $S_{K}$. We identify a maximal point of $S\left(y_{1}\right)^{\text {top }}$ with a $K$-valued point of $S_{K}$. We say $S\left(y_{1}\right)^{\text {top }}$ is regular at a maximal point of it, if there exists a regular, open subscheme of $\bar{S}\left(y_{1}\right)$ which contains this point and whose topological space is contained in $S\left(y_{1}\right)^{\text {top }}$. As $S\left(y_{1}\right)^{\text {top }}$ is a dense, constructible subset of $\bar{S}\left(y_{1}\right)^{\text {top }}$, there exists a regular, open, dense subscheme $W\left(y_{1}\right)$ of $\bar{S}\left(y_{1}\right)$ such that $W\left(y_{1}\right)^{\text {top }} \subseteq S\left(y_{1}\right)^{\text {top }}$.

A point $y_{3} \in S(K)=S_{K}(K)$ belongs to $S\left(y_{1}\right)^{\text {top }}$ if and only if $y_{1}^{*}(\mathcal{D})$ and $y_{3}^{*}(\mathcal{D})$ are isomorphic. Let now $y_{2} \in S(K)=S_{K}(K)$ be a maximal point of $S\left(y_{1}\right)^{\text {top }}$. Let $i_{12}: y_{2}^{*}(\mathcal{D}) \rightarrow$ $y_{1}^{*}(\mathcal{D})$ be an isomorphism, cf. (a). We have $S\left(y_{2}\right)^{\text {top }}=S\left(y_{1}\right)^{\text {top }}$. For $i \in\{1,2\}$ let $I_{y_{i}}$ be the spectrum of the completion of the local ring of $S_{K}$ at $y_{i}$. We denote also by $y_{i}$ the factorization of $y_{i}$ through $I_{y_{i}}$. Let $I_{y_{i}} \times_{S_{K}} S\left(y_{1}\right)^{\text {top }}$ be the pullback of $S\left(y_{1}\right)^{\text {top }}$ to a constructible subset of $I_{y_{i}}^{\text {top }}$. Due to the versal property of $\mathcal{D}$ and the fact that $S$ is smooth over $\operatorname{Spec}\left(\mathbf{F}_{p}\right)$ of dimension $d(r-d)$, the local schemes $I_{y_{1}}$ and $I_{y_{2}}$ have dimension $d(r-d)$ and moreover there exists a unique isomorphism

$$
I_{12}: I_{y_{1}} \stackrel{\sim}{\longrightarrow} I_{y_{2}}
$$

such that the following two things hold (cf. [21, 4.8]; see [9, 2.4.4] for the equivalence of the categories of $p$-divisible groups over $I_{i}$ and over the formal completion of $I_{i}$ along $y_{i}$ ):

(i) we have $I_{12} \circ y_{1}=y_{2}: \operatorname{Spec}(K) \rightarrow I_{y_{2}}$;

(ii) there exists an isomorphism $I_{12}^{*}\left(\mathcal{D}_{I_{y_{2}}}\right) \stackrel{\sim}{\longrightarrow} \mathcal{D}_{I_{y_{1}}}$ that lifts $i_{12}$.

Due to (ii) the local geometries of $S\left(y_{1}\right)^{\text {top }}$ at $y_{1}$ and $y_{2}$ are the same. In other words, $I_{12}$ induces via restriction an isomorphism $J_{12}^{\text {top }}: I_{y_{1}} \times_{S_{K}} S\left(y_{1}\right)^{\text {top }} \stackrel{\sim}{\longrightarrow} I_{y_{2}} \times_{S_{K}} S\left(y_{1}\right)^{\text {top }}$ between constructible subsets. Any commutative $\mathbf{F}_{p}$-algebra of finite type is excellent, cf. [29, (34.A) and (34.B)]. So the morphism $I_{y_{i}} \rightarrow S_{K}$ is regular. From the last two sentences we get (cf. [29, (33)] for the regular part of (iv)) that:

(iii) the dimensions of $S\left(y_{1}\right)^{\text {top }}$ at $y_{1}$ and $y_{2}$ are the same;

(iv) if $S\left(y_{1}\right)^{\text {top }}$ is regular at $y_{2}$, then $S\left(y_{1}\right)^{\text {top }}$ is also regular at $y_{1}$.

By taking $y_{2}$ to be a maximal point of $W\left(y_{1}\right)^{\text {top }}$ and $y_{1}$ to be an arbitrary maximal point of $S\left(y_{1}\right)^{\text {top }}$, from (iii) we get that $S\left(y_{1}\right)^{\text {top }}$ is equidimensional and from (iv) we get that $S\left(y_{1}\right)^{\text {top }}$ is regular at all its maximal points. So $\bar{S}\left(y_{1}\right)$ is also equidimensional. As $S\left(y_{1}\right)^{\text {top }}$ is a constructible subset of $\bar{S}\left(y_{1}\right)^{\text {top }}$, from the last two sentences we get that $S\left(y_{1}\right)^{\text {top }}$ is the underlying topological space of an equidimensional, regular, open subscheme $S\left(y_{1}\right)$ of $\bar{S}\left(y_{1}\right)$. Thus $S\left(y_{1}\right)$ is a reduced, 
locally closed subscheme of $S_{K}$. Let

$$
J_{12}: I_{y_{1}} \times_{S_{K}} S\left(y_{1}\right) \stackrel{\sim}{\longrightarrow} I_{y_{2}} \times_{S_{K}} S\left(y_{1}\right)
$$

be the isomorphism of reduced schemes defined by $I_{12}$ (or $J_{12}^{\text {top }}$ ).

Let $\mathcal{S}_{K}(\mathcal{D})$ be the set of reduced, locally closed subschemes of $S_{K}$ that are of the form $S\left(y_{1}\right)$ for some $y_{1} \in S(K)=S_{K}(K)$. Standard Galois descent shows that there exists a set $\mathcal{S}_{\mathbf{F}_{p}}(\mathcal{D})$ of reduced, locally closed subschemes of $S$ whose pullbacks to $S_{\overline{\mathbf{F}_{p}}}$ are the elements of $\mathcal{S}_{\overline{\mathbf{F}_{p}}}(\mathcal{D})$. If $L$ is an algebraically closed field that contains $K$ and if $y_{1}^{L}$ is the $L$-valued point of $S$ defined by $y_{1}$, then by the very definitions $S\left(y_{1}^{L}\right)=S\left(y_{1}\right)_{L}$. So we have natural pullback injective maps $\mathcal{S}_{K}(\mathcal{D}) \hookrightarrow \mathcal{S}_{L}(\mathcal{D})$ and $\mathcal{S}_{\mathbf{F}_{p}}(\mathcal{D}) \hookrightarrow \mathcal{S}_{L}(\mathcal{D})$. So $\mathcal{S}_{\mathbf{F}_{p}}(\mathcal{D})$ and $\mathcal{S}_{K}(\mathcal{D})$ 's define a stratification $\mathcal{S}(\mathcal{D})$ of $S$ in the sense of 2.1.1. So as each $S\left(y_{1}\right)$ is a regular and equidimensional $\operatorname{Spec}(K)$-scheme, (b) holds.

Let $v, n \in \mathbf{N}$ be as in 5.1.1(c) for $r_{1}=r_{2}:=r$. Let $\tilde{n}:=\max \{q, l, n\}$. For $m \in\{\tilde{n}, \tilde{n}+v\}$ let $\mathbf{I}_{m}\left(y_{1}\right)$ be the $\bar{S}\left(y_{1}\right)$-scheme that parametrizes isomorphisms between $\mathcal{D}_{\bar{S}\left(y_{1}\right)}$ and the pullback of $y_{1}^{*}(\mathcal{D})$ through the natural morphism $\bar{S}\left(y_{1}\right) \rightarrow \operatorname{Spec}(K)$. We consider the natural truncation morphism $\mathbf{T}_{\tilde{n}, v}: \mathbf{I}_{\tilde{n}+v}\left(y_{1}\right) \rightarrow \mathbf{I}_{\tilde{n}}\left(y_{1}\right)$ of $\bar{S}\left(y_{1}\right)$-schemes.

Let $\mathbf{I}_{\tilde{n}, v}\left(y_{1}\right)$ be the minimal reduced, closed subscheme of $\mathbf{I}_{\tilde{n}}\left(y_{1}\right)_{\text {red }}$ through which the reduced morphism defined by $\mathbf{T}_{\tilde{n}, v}$ factors. As $\tilde{n} \geqslant l$, from (a) we get that $\mathbf{I}_{\tilde{n}, v}\left(y_{1}\right)$ is in fact an $S\left(y_{1}\right)$-scheme. The resulting morphism

$$
m_{\tilde{n}, v}\left(y_{1}\right): \mathbf{I}_{\tilde{n}, v}\left(y_{1}\right) \rightarrow S\left(y_{1}\right)
$$

is surjective, cf. the definition of $S\left(y_{1}\right)$. To prove (c) and (d), it suffices to show that $S\left(y_{1}\right)$ is an affine $\bar{S}\left(y_{1}\right)$-scheme and that $\mathbf{I}_{\tilde{n}, v}\left(y_{1}\right)$ is a regular scheme that is finite, flat over $S\left(y_{1}\right)$. It suffices to check this under the extra assumption that $S$ is affine. So the schemes $S_{K}, \bar{S}\left(y_{1}\right), \mathbf{I}_{\tilde{n}}\left(y_{1}\right)$, and $\mathbf{I}_{\tilde{n}, v}\left(y_{1}\right)$ are also affine.

We check that the surjective morphism $m_{\tilde{n}, v}\left(y_{1}\right): \mathbf{I}_{\tilde{n}, v}\left(y_{1}\right) \rightarrow S\left(y_{1}\right)$ is quasi-finite above any point $y_{\text {gen }}$ of $S\left(y_{1}\right)$ of codimension 0 . Let $F_{\text {gen }}$ and $I_{\text {gen }}$ be the fibres over $y_{\text {gen }}$ of $\mathbf{I}_{\tilde{n}, v}\left(y_{1}\right)$ and $\mathbf{I}_{\tilde{n}+v}\left(y_{1}\right)$ (respectively). We show that the assumption that $F_{\text {gen }}$ is not of dimension 0 leads to a contradiction. This assumption implies that the image of $I_{\text {gen }}$ in $F_{\text {gen }}$ contains an open, dense subscheme of $F_{\text {gen }}$ of positive dimension. We get the existence of an algebraically closed field $L$ that contains the residue field of $y_{\text {gen }}$ and such that the number of automorphisms of $y_{1}^{*}(\mathcal{D})_{L}\left[p^{\tilde{n}}\right]$ that lift to automorphisms of $y_{1}^{*}(\mathcal{D})_{L}\left[p^{\tilde{n}+v}\right]$ is infinite. From this and 5.1.1(c) we get that the image of $\operatorname{Aut}\left(y_{1}^{*}(\mathcal{D})_{L}\right)=\operatorname{Aut}\left(y_{1}^{*}(\mathcal{D})\right)$ in $\operatorname{Aut}\left(y_{1}^{*}(\mathcal{D})_{L}\left[p^{\tilde{n}}\right]\right)$ is infinite. $\operatorname{But} \operatorname{Aut}\left(y_{1}^{*}(\mathcal{D})\right)$ is a $\mathbf{Z}_{p}$-algebra of finite rank and so this image is finite. Contradiction.

So $F_{\text {gen }}$ has dimension 0 . Thus there exists an open, dense subscheme $U\left(y_{1}\right)$ of $S\left(y_{1}\right)$ such that the reduced $\operatorname{Spec}(K)$-scheme of finite type $\mathbf{I}_{\tilde{n}, v}\left(y_{1}\right) \times_{\bar{S}\left(y_{1}\right)} U\left(y_{1}\right)$ is regular as well as (cf. [18, Ch. IV, (9.6.1) and (11.1.1)] and the surjectivity of $\left.m_{\tilde{n}, v}\right)$ finite, flat over $U\left(y_{1}\right)$. From (ii) and constructions we get the existence of an isomorphism of $S_{K}$-schemes

$$
K_{12}: I_{y_{1}} \times_{S_{K}} \mathbf{I}_{\tilde{n}, v}\left(y_{1}\right) \stackrel{\sim}{\longrightarrow} I_{y_{2}} \times_{S_{K}} \mathbf{I}_{\tilde{n}, v}\left(y_{1}\right)
$$

such that we have $\left(1_{I_{y_{2}}} \times_{S_{K}} m_{\tilde{n}, v}\left(y_{1}\right)\right) \circ K_{12}=J_{12} \times_{S\left(y_{1}\right)} m_{\tilde{n}, v}\left(y_{1}\right)$. In particular, we get:

(v) the morphism $m_{\tilde{n}, v}\left(y_{1}\right)$ is finite and flat above an open subscheme of $S\left(y_{1}\right)$ that contains $y_{1}$ if and only if it is so above an open subscheme of $S\left(y_{1}\right)$ that contains $y_{2}$.

As in the above part that pertains to local geometries, the existence of such isomorphisms $K_{12}$ of $S_{K}$-schemes implies that $\mathbf{I}_{\tilde{n}, v}\left(y_{1}\right)$ is regular and equidimensional. From (v) and the existence of $U\left(y_{1}\right)$ we get that $\mathbf{I}_{\tilde{n}, v}\left(y_{1}\right)$ is a finite, flat $S\left(y_{1}\right)$-scheme. From this and the fact that $\mathbf{I}_{\tilde{n}, v}\left(y_{1}\right)$ 
is a regular subscheme of $\mathbf{I}_{\tilde{n}}\left(y_{1}\right)$, we get that (d) holds for $\tilde{n}$ (and so also for $q \leqslant \tilde{n}$ ) and that $\mathbf{I}_{\tilde{n}, v}\left(y_{1}\right)$ is the normalization of $S\left(y_{1}\right)$ in the ring of fractions of $\mathbf{I}_{\tilde{n}, v}\left(y_{1}\right)$. As $\mathbf{I}_{\tilde{n}, v}\left(y_{1}\right)$ is affine and $m_{\tilde{n}, v}\left(y_{1}\right)$ is a finite, surjective morphism, from the Chevalley theorem of [16, Ch. II, (6.7.1)] we get that $S\left(y_{1}\right)$ is affine. So (c) holds.

\subsubsection{Ultimate stratifications}

Let $N \geqslant 3$ and $\mathcal{A}_{d, 1, N}$ be as in 1.5 . Let $\left(\mathcal{A}, \lambda_{\mathcal{A}}\right)$ be the universal principally polarized abelian scheme over $\mathcal{A}_{d, 1, N}$. We have:

(a) There exists a stratification $\mathcal{S}_{d, N}$ of $\mathcal{A}_{d, 1, N}$ defined by the following property: two geometric points $y_{1}$ and $y_{2}$ of $\mathcal{A}_{d, 1, N}$ with values in the same algebraically closed field $K$, factor through the same stratum of $\mathcal{S}_{d, N}$ if and only if the principally quasi-polarized p-divisible groups of $y_{1}^{*}\left(\mathcal{A}, \lambda_{\mathcal{A}}\right)$ and $y_{2}^{*}\left(\mathcal{A}, \lambda_{\mathcal{A}}\right)$, are isomorphic.

(b) The stratification $\mathcal{S}_{d, N}$ of $\mathcal{A}_{d, 1, N}$ satisfies the purity property and its strata are regular and equidimensional.

(c) Let $q \in \mathbf{N}$. Let $K$ be as in (i). Then for any stratum $S_{0}$ of the stratification $\mathcal{S}_{d, N}$ that is a subscheme of $\mathcal{A}_{d, 1, N_{K}}$, there exists a regular scheme $S_{0}[q]$ that is finite, flat over $S_{0}$ and such that the pullback to $S_{0}[q]$ of the principally quasi-polarized truncated BarsottiTate group of level $q$ of $\left(\mathcal{A}, \lambda_{\mathcal{A}}\right)$ is constant, i.e. it is the pullback to $S_{0}[q]$ of a principally quasi-polarized truncated Barsotti-Tate group of level q over $\operatorname{Spec}(K)$.

The proofs of (a) to (c) are the same as those of 5.3.1(b) to (d), cf. 3.2.5. We only have to add that the use of $[21,4.8]$ in the proof of 5.3.1(b) has to be substituted by the well known fact that the formal deformation spaces of a principally polarized abelian variety over $\operatorname{Spec}(K)$ and of its principally quasi-polarized $p$-divisible group, are naturally identified (cf. the Serre-Tate deformation theory of [23, Ch. 1]).

Let $\operatorname{Sch}_{\text {red }} \mathbf{F}_{p}$ be the category of reduced $\operatorname{Spec}\left(\mathbf{F}_{p}\right)$-schemes endowed with the étale topology. Let $\mathcal{A}_{d, 1}$ be the moduli stack over $\operatorname{Sch}_{\text {red }}^{\mathbf{F}_{p}}$ of principally polarized abelian schemes of relative dimension $d$ (see [13, Ch. I, $\S 4$, p. 17 and 4.3]). The stratification $\mathcal{S}_{d, N}$ descends to a stratification $\mathcal{S}_{d}$ of $\mathcal{A}_{d, 1}$. As we did not formalize stratifications of stacks, we describe $\mathcal{S}_{d}$ directly as follows.

We fix a principally quasi-polarized $p$-divisible group $\mathcal{T}:=\left(D, \lambda_{D}\right)$ over $\operatorname{Spec}(k)$ of height $r=2 d$. The objects of $\mathcal{A}_{d, 1}$ are principally polarized abelian schemes over reduced $\operatorname{Spec}\left(\mathbf{F}_{p}\right)$ schemes. The substack $\mathcal{A}_{d, 1}(\mathcal{T})$ of $\mathcal{A}_{d, 1}$ associated to $\mathcal{T}$ is the full subcategory of $\mathcal{A}_{d, 1}$ whose objects are principally polarized abelian schemes over reduced $\operatorname{Spec}\left(\mathbf{F}_{p}\right)$-schemes with the property that all principally quasi-polarized $p$-divisible groups obtained from them via pullbacks through points with values in the same algebraically closed field $K$ that contains $k$, are isomorphic to $\mathcal{T} \times_{\operatorname{Spec}(k)} \operatorname{Spec}(K)$.

We refer to $\mathcal{S}_{d}$ (respectively to $\mathcal{S}_{d, N}$ ) as the ultimate stratification of $\mathcal{A}_{d, 1}$ (respectively of $\left.\mathcal{A}_{d, 1, N}\right)$.

Let $\mathcal{A}_{d, 1}(\mathcal{T})_{k}:=\mathcal{A}_{d, 1}(\mathcal{T}) \times{ }_{\operatorname{Sch}_{\text {red }}}^{\mathbf{F}_{p}} \operatorname{Sch}_{\text {red }}^{k}$ and $\mathcal{A}_{d, 1_{k}}:=\mathcal{A}_{d, 1} \times{ }_{\operatorname{Sch}_{\text {red }}}^{\mathbf{F}_{p}} \operatorname{Sch}_{\text {red }}^{k}$, where $\operatorname{Sch}_{\text {red }}^{k}$ is the full subcategory of $\operatorname{Sch}_{\text {red }}^{\mathbf{F}_{p}}$ formed by reduced $\operatorname{Spec}(k)$-schemes. The pullback of $\mathcal{A}_{d, 1}(\mathcal{T})_{k}$ via the 1-morphism $\mathcal{A}_{d, 1, N_{k}} \rightarrow \mathcal{A}_{d, 1_{k}}$ is the stratum of $\mathcal{S}_{d, N}$ that is a subscheme of $\mathcal{A}_{d, 1, N_{k}}$ and that corresponds naturally to $\mathcal{T}$. Using this it can be easily checked that $\mathcal{A}_{d, 1}(\mathcal{T})_{k}$ is a separated, algebraic stack over $\mathrm{Sch}_{\mathrm{red}}^{k}$ in the similar sense as of [13, Ch. I, §4, 4.6 and 4.8] but worked out using only reduced $\operatorname{Spec}(k)$-schemes.

The following proposition, to which we refer as the integral Manin problem for Siegel modular varieties (see [28, p. 76] and [39, p. 98] for the original Manin problem), implies that $\mathcal{A}_{d, 1}(\mathcal{T})$ is a non-empty category.

$4^{\text {e }}$ SÉRIE - TOME $39-2006-\mathrm{N}^{\circ} 2$ 
5.3.3. Proposition. - Let $\operatorname{Spec}(C)$ be a local, complete scheme whose residue field is $k=\bar{k}$. Then any principally quasi-polarized p-divisible group $\mathcal{T}_{C}^{\prime}$ over $\operatorname{Spec}(C)$ that lifts $\mathcal{T}$, is the one of a principally polarized abelian scheme over $\operatorname{Spec}(C)$.

Proof. - We first show that $\mathcal{T}$ is associated to a principally polarized abelian variety $\left(A, \lambda_{A}\right)$ over $\operatorname{Spec}(k)$. Let $\left(M, \varphi, \lambda_{M}\right)$ be the principally quasi-polarized Dieudonné module of $\mathcal{T}$. Let $\widetilde{A}$ be an abelian variety $\widetilde{A}$ over $\operatorname{Spec}(k)$ whose $F$-isocrystal is $\left(M\left[\frac{1}{p}\right], \varphi\right)$, cf. [39, p. 98]. Based on [32, $\$ 23$, Cor. 1], up to an isogeny, we can choose $\widetilde{A}$ such that it has a principal polarization $\lambda_{\widetilde{A}}$. The principally quasi-polarized Dieudonné module of $\left(\widetilde{A}, \lambda_{\widetilde{A}}\right)$ is of the form $\left(\widetilde{M}, \varphi, \lambda_{\widetilde{M}}\right)$, where $\widetilde{M}$ is a certain $W(k)$-lattice of $M\left[\frac{1}{p}\right]$.

5.3.3.1. LEMMA. - If $\lambda_{1}$ and $\lambda_{2}$ are two principal quasi-polarizations of $\left(M\left[\frac{1}{p}\right], \varphi\right)$, then the triples $\left(M\left[\frac{1}{p}\right], \varphi, \lambda_{1}\right)$ and $\left(M\left[\frac{1}{p}\right], \varphi, \lambda_{2}\right)$ are isomorphic.

Proof. - It suffices to prove the lemma under the assumption that there exists $\alpha \in\left[0, \frac{1}{2}\right] \cap \mathbf{Q}$ such that all slopes of $\left(M\left[\frac{1}{p}\right], \varphi\right)$ are $\alpha$ and $1-\alpha$, cf. Dieudonné's classification of $F$-isocrystals over $k$ (see [28, §2]) and [39, p. 98]. Let $i \in\{1,2\}$.

We first consider the case when $\alpha \neq \frac{1}{2}$; so $\alpha \neq 1-\alpha$. Let $M\left[\frac{1}{p}\right]=M_{\alpha} \oplus M_{1-\alpha}$ be the direct sum decomposition that is normalized by $\varphi$ and such that for $\beta \in\{\alpha, 1-\alpha\}$ all slopes of $\left(M_{\beta}, \varphi\right)$ are $\beta$. We have $\lambda_{i}\left(M_{\beta}, M_{\beta}\right)=0$ and the bilinear form $\lambda_{i, \beta}: M_{\beta} \otimes_{B(k)} M_{1-\beta} \rightarrow B(k)$ induced naturally by $\lambda_{i}$, is non-degenerate. But $\lambda_{i, 1-\alpha}$ is determined by $\lambda_{i, \alpha}$. Thus $\lambda_{i}$ is uniquely determined by the isomorphism $j_{i}:\left(M_{\alpha}, \varphi\right) \stackrel{\sim}{\sim}\left(M_{1-\alpha}^{*}, p 1_{M_{1-\alpha}^{*}} \varphi\right)$ defined naturally by $\lambda_{i, \alpha}$ via the rule $j_{i}(x)(y)=\lambda_{i}(x, y)=\lambda_{i, \alpha}(x, y)$, where $x \in M_{\alpha}$ and $y \in M_{1-\alpha}$. Let $f_{12}:=j_{1}^{-1} j_{2}:\left(M_{\alpha}, \varphi\right) \stackrel{\sim}{\longrightarrow}\left(M_{\alpha}, \varphi\right)$. The automorphism $e_{12}:=f_{12} \oplus 1_{M_{1-\alpha}}$ of $\left(M\left[\frac{1}{p}\right], \varphi\right)=$ $\left(M_{\alpha}, \varphi\right) \oplus\left(M_{1-\alpha}, \varphi\right)$ takes $\lambda_{2}$ into $\lambda_{1}$, i.e. for $x, y \in M\left[\frac{1}{p}\right]$ we have an identity $\lambda_{2}(x, y)=$ $\lambda_{1}\left(e_{12}(x), e_{12}(y)\right)$.

Let now $\alpha$ be $\frac{1}{2}$. As $\alpha=\frac{1}{2}$, the $F$-isocrystal $\left(M\left[\frac{1}{p}\right], \varphi\right)$ over $k$ is a direct sum of simple $F$-isocrystals over $k$ of rank 2 . Using the standard argument that shows that any two nondegenerate, symmetric, bilinear forms on an even dimensional complex vector space are isomorphic, we get that both $\left(M\left[\frac{1}{p}\right], \varphi, \lambda_{1}\right)$ and $\left(M\left[\frac{1}{p}\right], \varphi, \lambda_{2}\right)$ are direct sums of principally quasi-polarized $F$-isocrystals over $k$ of rank 2 . Thus we can assume $r_{M}=2$ (i.e. $d=1$ ). But in this case the lemma is trivial (for instance, cf. [27, pp. 35-36]).

Based on the lemma, it suffices to prove the proposition under the extra hypothesis that $\lambda_{M}=\lambda_{\widetilde{M}}$. From the classical Dieudonné theory we get directly the next property.

5.3.3.2. THE ISOGENY PROPERTY.-There exists a unique principally polarized abelian variety $\left(A, \lambda_{A}\right)$ over $\operatorname{Spec}(k)$ that is $\mathbf{Z}\left[\frac{1}{p}\right]$-isogenous to $\left(\widetilde{A}, \lambda_{\widetilde{A}}\right)$ and whose principally quasipolarized Dieudonné module is identifiable under this $\mathbf{Z}\left[\frac{1}{p}\right]$-isogeny with $\left(M, \varphi, \lambda_{M}\right)$.

So $\mathcal{T}$ is the principally quasi-polarized $p$-divisible group of $\left(A, \lambda_{A}\right)$. From the Serre-Tate deformation theory (see [23, Ch. 1]) and the Grothendieck algebraization theorem (see [17, Ch. III, Thm. (5.4.5)]), we easily get the existence of a principally polarized abelian scheme over $\operatorname{Spec}(C)$ whose principally quasi-polarized $p$-divisible group is $\mathcal{T}_{C}^{\prime}$.

5.3.4. Remarks. - (a) If $d \geqslant 2$, then the stratification $\mathcal{S}_{d, N}$ has a class which is not a set (for $d \geqslant 3$ this follows from 4.5.4).

(b) Let $q \in \mathbf{N}$. Let $\mathcal{S}_{d, N, q}$ be the stratification of $\mathcal{A}_{d, 1, N}$ defined by the rule: two geometric points $y_{1}$ and $y_{2}$ of $\mathcal{A}_{d, 1, N}$ with values in the same algebraically closed field, factor through the same stratum of $\mathcal{S}_{d, N, q}$ if and only if the principally quasi-polarized truncated Barsotti-Tate 
groups of level $q$ of $y_{1}^{*}\left(\mathcal{A}, \lambda_{\mathcal{A}}\right)$ and $y_{2}^{*}\left(\mathcal{A}, \lambda_{\mathcal{A}}\right)$, are isomorphic. The strata of $\mathcal{S}_{d, N, q}$ are regular and equidimensional (one argues this in a way similar to the proof of 5.3.1; instead of [21, 4.8] one has to use a principal quasi-polarized version of [21, 4.7 and 4.8] and the Serre-Tate deformation theory). For $q \geqslant T(d)$ we have $\mathcal{S}_{d, N, q}=\mathcal{S}_{d, N}$, cf. 3.2.5. The case $q=1$ was first studied by Ekedahl and Oort, cf. [34, §1]. The strata of $\mathcal{S}_{d, N, 1}$ are quasi-affine, cf. [34, 1.2]. As each stratum of $\mathcal{S}_{d, N, q}$ is a locally closed subscheme of a stratum of $\mathcal{S}_{d, N, 1}$, the strata of $\mathcal{S}_{d, N, q}$ are also quasi-affine. For $1 \leqslant q<T(d)$, we do not know when the stratification $\mathcal{S}_{d, N, q}$ satisfies the purity property.

(c) The existence of the ultimate stratifications $\mathcal{S}_{d}$ and $\mathcal{S}_{d, N}$, though of foundation, is only a first step toward the solution of the below Main Problem. Due to the importance of Main Problem, we will state a general form of it, even if in this paper we do not formalize specializations of latticed $F$-isocrystals with a group (such specializations are standard for $p$-divisible groups; see also 3.2.7). To be short, we state Main Problem only in a context that involves tensors but no principal bilinear quasi-polarizations.

Main Problem. - Let $\left(M, \varphi, G,\left(t_{\alpha}\right)_{\alpha \in \mathcal{J}}\right)$ be a latticed $F$-isocrystal with a group and an emphasized family of tensors over $k$ such that the $W$-condition holds for $(M, \varphi, G)$ (see 2.2.1(b) and (d)). List using families all isomorphism classes of $\left(M, g \varphi, G,\left(t_{\alpha}\right)_{\alpha \in \mathcal{J})}\right.$ 's (where $g \in G(W(k)))$ and decide which such classes specialize to which other.

\subsection{On the specialization theorem}

Let $S$ be an integral $\operatorname{Spec}\left(\mathbf{F}_{p}\right)$-scheme. We take $k$ to be an algebraic closure of the field of fractions $k_{S}$ of $S$. Let $\mathfrak{C}$ be an $F$-crystal over $S$. Let $h_{\mathfrak{C}} \in \mathbf{N} \cup\{0\}$ be as in 2.1; the $h$-number of any pullback of $\mathfrak{C}$ via a geometric point of $S$ is at most $h_{\mathfrak{C}}$. Let $\mathcal{N}$ be the Newton polygon of $\mathfrak{C}_{k}$. Let

$$
U^{\text {top }}:=\left\{x \in S^{\text {top }} \mid x^{*}(\mathfrak{C}) \text { has Newton polygon } \mathcal{N}\right\} .
$$

We recall that Grothendieck proved that for any geometric point $y$ of $S$ the Newton polygon of $y^{*}(\mathfrak{C})$ is above $\mathcal{N}$ (see [19, Appendice]) and that Katz added that moreover there exists an open subscheme $U$ of $S$ such that the notations match, i.e. $U^{\text {top }}$ is the topological space underlying $U$ (see [22, 2.3.1 and 2.3.2]).

We give another proof of the existence of $U$ using Grothendieck's result. This result implies that if $x \in U^{\text {top }}$, then all points of the spectrum of the local ring of $x$ in $S$ belong to $U^{\text {top }}$. To show the existence of $U$, it is enough to show that there exists a non-empty open subscheme $U^{\prime}$ of $S$ such that $U^{\prime \text { top }} \subseteq U^{\text {top }}$. The argument for this goes as follows. The existence of such open subschemes $U^{\prime}$ implies that $U^{\text {top }}$ is an ind-constructible subset of $S^{\text {top }}$, cf. [18, Ch. IV, (9.2.1) and (9.2.3)]. Based on this and the above part that pertains to $x \in U^{\text {top }}$, from [18, Ch. IV, Thm. (1.10.1)] we get that each point of $U^{\text {top }}$ is an interior point of $U^{\text {top }}$. Thus $U^{\text {top }}$ is an open subset of $S^{\text {top }}$ and therefore $U$ exists.

Let $\mathfrak{C}_{0}$ be a Dieudonné-Fontaine $p$-divisible object over $\mathbf{F}_{p}$ of Newton polygon $\mathcal{N}$. Let $h_{0}$ be the $h$-number of $\mathfrak{C}_{0}$ and let $r_{0}$ be the rank of $\mathfrak{C}_{0}$. Let $i: \mathfrak{C}_{0 k} \rightarrow \mathfrak{C}_{k}$ be an isogeny. Let $l \in \mathbf{N}$ be such that $p^{l}$ annihilates Coker $(i)$. Let $v:=\max \left\{v\left(r_{0}, r_{0}, h_{0}, b\right) \mid b \in S\left(0, h_{\mathfrak{C}}\right)\right\}$ and $n:=\max \left\{1, n\left(r_{0}, r_{0}, h_{0}, b\right) \mid b \in S\left(0, h_{\mathfrak{C}}\right)\right\}$ be defined using the numbers of 5.1.1(b).

Let $i(n+v+l)$ be the reduction of $i \bmod p^{n+v+l}$; it is a morphism of $\mathcal{M}\left(W_{n+v+l}(k)\right)$ whose cokernel is annihilated by $p^{l}$. From 2.8.3 (see 2.8.3(a) applied with $\left(V_{1}, V, q\right)=$ $\left.\left(k, k_{S}, n+v+l\right)\right)$, we get that there exists a finite field extension $k_{\widetilde{S}}$ of $k_{S}$ such that $i(n+v+l)$ is the pullback of a morphism $i(n+v+l)_{k_{\tilde{S}}}$ of $\mathcal{M}\left(W_{n+v+l}\left(k_{\widetilde{S}}\right)\right)$ whose cokernel is annihilated by $p^{l}$. Let $\widetilde{S}$ be the normalization of $S$ in $k_{\widetilde{S}}$ (the notations match, i.e. $k_{\widetilde{S}}$ is the field of fractions 
of $\widetilde{S}$ ). The continuous map $\widetilde{S}^{\text {top }} \rightarrow S^{\text {top }}$ is proper, cf. the going-up theorem of [29, (5.E)]. So if there exists an open, dense subscheme $\widetilde{U}^{\prime}$ of $\widetilde{S}$ with the property that $\widetilde{U}^{\text {top }}$ maps into $U^{\text {top }}$, then we can take $U^{\prime}$ to be the complement in $S$ of the image of $\widetilde{S}^{\text {top }} \backslash \widetilde{U}^{\text {top }}$ in $S^{\text {top }}$. Thus it suffices to consider the case when $k_{\widetilde{S}}=k_{S}$. Let $U^{\prime}$ be an open subscheme of $S$ such that we have a morphism

$$
i_{U^{\prime}}(n+v+l): \mathbf{E}\left(\mathfrak{C}_{0} ; W_{n+v+l}\left(U^{\prime}\right)\right) \rightarrow \mathbf{E}\left(\mathfrak{C} ; W_{n+v+l}\left(U^{\prime}\right)\right)
$$

of $\mathcal{M}\left(W_{n+v+l}\left(U^{\prime}\right)\right)$ that extends $i(n+v+l)_{k_{S}}$ and that has a cokernel annihilated by $p^{l}$, cf. 2.8.3(b). For an arbitrary geometric point $z: \operatorname{Spec}(K) \rightarrow U^{\prime}$, the reduction $\bmod p^{n+l}$ of the pullback morphism $z^{*}\left(i_{U^{\prime}}(n+v+l)\right)$ lifts to a morphism $i_{z}: \mathfrak{C}_{0 K} \rightarrow z^{*}(\mathfrak{C})$ of $F$-crystals over $K$ (cf. 5.1.1(b), 5.1.2, and the definitions of $v$ and $n$ ). As the cokernel of the reduction $\bmod p^{n+l}$ of $i_{z}$ is annihilated by $p^{l}$ and as $n \geqslant 1$ (by reasons of ranks) the morphism $i_{z}$ is injective and thus an isogeny. Thus $z^{*}(\mathfrak{C})$ has Newton polygon $\mathcal{N}$. So $U^{\text {top }} \subseteq U^{\text {top }}$. This ends the argument for the existence of $U^{\prime}$ and so also of $U$.

\section{Proof of Main Theorem B}

Let $S$ be a reduced $\operatorname{Spec}\left(\mathbf{F}_{p}\right)$-scheme. Let $\mathfrak{C}$ be an $F$-crystal over $S$. Let $\mathcal{S}(\mathfrak{C})$ be the Newton polygon stratification of $S$ defined by $\mathfrak{C}$, cf. [22, 2.3.1 and 2.3.2]. The stratification $\mathcal{S}(\mathfrak{C})$ is of finite type and locally in the Zariski topology of $S$ has a finite number of strata. The main goal of this Section is to prove Main Theorem B stated in 1.6, i.e. to prove that $\mathcal{S}(\mathfrak{C})$ satisfies the purity property (see 6.2). In 6.1 we capture the very essence of Main Theorem B for the case when $S$ is an integral, locally noetherian scheme. In 6.3 we include two remarks on the connection between 6.1 and a result of de Jong and Oort and on Newton polygon stratifications defined by certain reductions modulo powers of $p$ of $F$-crystals. We will use the notations of 2.8.2.

6.1. TheOREM. - Suppose $S$ is integral and locally noetherian. Let $U$ be the maximal open subscheme of $S$ with the property that the Newton polygons of pullbacks of $\mathfrak{C}$ through geometric points of $U$ are all equal (see 5.4). Then $U$ is an affine $S$-scheme.

Proof. - It suffices to prove this under the extra assumptions that $S=\operatorname{Spec}(R)$ is affine and that the underlying $R$-module of $\mathbf{E}\left(\mathfrak{C} ; W_{1}(S)\right)$ is free. Let $R_{U}$ be the $R$-algebra of global functions of $U$. We have to show that $U$ is affine, i.e. the natural and functorial morphism $f_{U}: U \rightarrow \operatorname{Spec}\left(R_{U}\right)$ is an isomorphism. This statement is local in the faithfully flat topology of $S$ and thus we can assume that $S$ is local. Let $\widehat{R}$ be the completion of $R$ and let $\widehat{S}:=\operatorname{Spec}(\widehat{R})$. As $\widehat{S}$ is a faithfully flat $S$-scheme, to show that $U$ is affine (i.e. $f_{U}$ is an isomorphism) it suffices to show that $U \times_{S} \widehat{S}$ is affine (i.e. $f_{U} \times_{S} \widehat{S}=f_{U \times_{S} \widehat{S}}$ is an isomorphism). Let $\widehat{S}_{1}=\operatorname{Spec}\left(\widehat{R}_{1}\right), \ldots, \widehat{S}_{j}=\operatorname{Spec}\left(\widehat{R}_{j}\right)$ be the irreducible components of the reduced scheme of $\widehat{S}$ (here $j \in \mathbf{N}$ ); they are spectra of local, complete, integral, noetherian $\mathbf{F}_{p}$-algebras. The scheme $U \times_{S} \widehat{S}$ is affine if and only if the irreducible components $U \times_{S} \widehat{S}_{1}, \ldots, U \times_{S} \widehat{S}_{j}$ of the reduced scheme of $U \times{ }_{S} \widehat{S}$ are all affine (cf. [16, Ch. II, Cor. (6.7.3)]). So to prove the theorem we can assume $R=\widehat{R}=\widehat{R}_{1}$. As $R$ is a local, complete ring, it is also excellent (cf. [29, (34.B)]). Thus the normalization $S^{\mathrm{n}}$ of $S$ is a finite $S$-scheme. So $S^{\mathrm{n}}$ is a semilocal, complete, integral, normal scheme. This implies that $S^{\mathrm{n}}$ is local. But $U$ is affine if and only if $U \times_{S} S^{\mathrm{n}}$ is affine, cf. 2.9.2. Thus to prove the Theorem, we can also assume $S$ is normal; so $S=S^{\text {n }}$.

We emphasize that for the rest of the proof we will only use the fact that $S$ is an integral, normal, excellent, affine scheme (but not necessarily local and thus not necessarily complete). We group the main steps into distinct (and numbered) sections. 


\subsubsection{Notations and two operations}

Let $k_{S}, k, h_{\mathfrak{C}}, \mathcal{N}, \mathfrak{C}_{0}, h_{0}, r_{0}, v$, and $n$ be as in 5.4. So $\mathcal{N}$ is the Newton polygon of pullbacks of $\mathfrak{C}_{U}$ via geometric points of $U, \mathfrak{C}_{0}$ is a Dieudonné-Fontaine $p$-divisible object over $\mathbf{F}_{p}$ that has Newton polygon $\mathcal{N}, r_{0}$ is the rank of $\mathfrak{C}_{0}$, etc. Let $q_{0}:=r_{0}$ !. Below all pullbacks to $\operatorname{Spec}(k)$ of $F$-crystals are via the natural dominant morphisms $\operatorname{Spec}(k) \rightarrow \operatorname{Spec}\left(\mathbf{F}_{p}\right)$ and $\operatorname{Spec}(k) \rightarrow S$.

We consider the following two replacement operations $(\mathcal{R} 1)$ and $(\mathcal{R} 2)$ of the triple $(S, U, \mathfrak{C})$ by a new triple $(\widetilde{S}, \widetilde{U}, \widetilde{\mathfrak{C}})$. For both operations $\widetilde{S}$ is an integral, normal, affine $S$-scheme of finite type, $\widetilde{\mathfrak{C}}$ is $\mathfrak{C}_{\widetilde{S}}$, and

$(\mathcal{R} 1)$ either $(\widetilde{S}, \widetilde{U})$ is the normalization of $(S, U)$ in a finite field extension of $k_{S}$

$(\mathcal{R} 2)$ or $U$ is an open subscheme of $\widetilde{S}$ and $\widetilde{U}:=U$ is $U \times_{S} \widetilde{S}$.

The scheme $\widetilde{S}$ is also excellent, cf. [29, (34.B)]. Moreover, $U$ is affine if and only if $\widetilde{U}$ is affine (in connection with $(\mathcal{R} 1)$, cf. 2.9.2). So in what follows we will often perform one of these two operations in order to simplify the setting and to eventually end up with a situation where in fact we have $U=S$. By performing $(\mathcal{R} 1)$, we can assume that $R$ is an $\mathbf{F}_{p^{q_{0}}}$-algebra (i.e. $\mathbf{F}_{p^{q_{0}}} \hookrightarrow R$ ).

Let $V$ be a local ring of $U$ that is a discrete valuation ring. Let $V_{2}$ be a complete discrete valuation ring that is a faithfully flat $V$-algebra, and that has an algebraically closed residue field $k_{2}$. Let $V_{1}:=V_{2}^{\text {perf }}$. We fix an isomorphism $V_{2} \stackrel{\sim}{\longrightarrow} k_{2}[[w]]$ and we view it as an identification under which $V_{2}$ and $V_{1}$ become $k_{2}$-algebras. Let $\Phi_{2}$ be the Frobenius endomorphism of $W\left(k_{2}\right)[[w]]$ that takes $w$ into $w^{p}$ and is compatible with $\sigma_{k_{2}}$.

6.1.2. Key LEMMA. - There exists a number $l \in \mathbf{N}$ that is greater than $\max \left\{h_{0} r_{0}^{2}, h_{\mathfrak{C}}\right\}$, that depends only on $\mathfrak{C}_{0}$ and $h_{\mathfrak{C}}$ but not on $V$, and such that there exists an isogeny $i_{V_{1}}: \mathfrak{C}_{0 V_{1}} \rightarrow \mathfrak{C}_{V_{1}}$ of $F$-crystals over $\operatorname{Spec}\left(V_{1}\right)$ whose cokernel is annihilated by $p^{l}$.

Proof. - We first show by induction on $r_{0} \in \mathbf{N}$ that there exists a number $\tilde{l}_{C} \in \mathbf{N}$ that does not depend on $V$ but only on $\mathfrak{C}_{0}$ and $h_{\mathfrak{C}}$ and such that we have an isogeny $i_{1}: \mathfrak{C}_{C V_{1}} \rightarrow \mathfrak{C}_{V_{1}}$ whose cokernel is annihilated by $p^{\tilde{l}_{C}}$, where $\mathfrak{C}_{C}$ is an $F$-crystal over $\operatorname{Spec}\left(k_{2}\right)$ and where the role of $\mathfrak{C}_{V_{1}}$ is that of the pullback to $\operatorname{Spec}\left(V_{1}\right)$ of an arbitrary $F$-crystal over $\operatorname{Spec}\left(V_{2}\right)$ of constant Newton polygon which depends only on $\mathcal{N}$.

Let $\alpha_{1}$ be the smallest slope of $\mathcal{N}$. Let $\tilde{l}_{1}:=-\left[-\alpha_{1}\left(r_{0}-1\right)\right]$. Theorem [22, 2.6.1] says that there exists an isogeny $i_{1}^{\prime}: \mathfrak{C}^{\prime} \rightarrow \mathfrak{C}_{V_{2}}$, where $\mathfrak{C}^{\prime}$ is an $\alpha_{1}$-divisible $F$-crystal over $\operatorname{Spec}\left(V_{2}\right)$. The $\alpha_{1}$-divisibility means that if $\left(M^{\prime}, \varphi^{\prime}, \nabla^{\prime}\right)$ is the evaluation of $\mathfrak{C}^{\prime}$ at the thickening defined by the closed embedding $\operatorname{Spec}\left(V_{2}\right) \hookrightarrow \operatorname{Spec}\left(W\left(k_{2}\right)[[w]]\right)$, then for all $u \in \mathbf{N}$ the $\Phi_{2}^{u}$-linear endomorphism $\left(\varphi^{\prime}\right)^{u}$ of $M^{\prime}$ is divisible by $p^{\left[u \alpha_{1}\right]}$. We can choose $\mathfrak{C}^{\prime}$ and $i_{1}^{\prime}$ such that Coker $\left(i_{1}^{\prime}\right)$ is annihilated by $p^{\tilde{l}_{1}}$, cf. [22, p. 153]. If $\alpha_{1}$ is the only slope of $\mathcal{N}$, then $\mathfrak{C}^{\prime}$ is the pullback of an $F$-crystal over $\operatorname{Spec}\left(k_{2}\right)$ (cf. [22, proof of 2.7.1]); so we can take $\tilde{l}_{C}$ to be $\tilde{l}_{1}$ and $i_{1}$ to be $i_{1}^{\prime}$. In particular, $\tilde{l}_{C}$ exists if $r_{0}=1$.

We now consider the case when $\mathcal{N}$ has at least two slopes. From [22, proof of 2.6.2] we get that we have a unique short exact sequence

$$
0 \rightarrow \mathfrak{C}_{1}^{\prime} \rightarrow \mathfrak{C}^{\prime} \rightarrow \mathfrak{C}_{2}^{\prime} \rightarrow 0
$$

of $F$-crystals over $\operatorname{Spec}\left(V_{2}\right)$ with the property that the Newton polygons of the pullbacks of $\mathfrak{C}_{1}^{\prime}$ (respectively of $\left.\mathfrak{C}_{2}^{\prime}\right)$ via geometric points of $\operatorname{Spec}\left(V_{2}\right)$ have all slopes equal to $\alpha_{1}$ (respectively have all slopes greater than $\alpha_{1}$ ). As $V_{1}$ is perfect, loc. cit. also proves that this short exact sequence has a unique splitting after we pull it back to $\operatorname{Spec}\left(V_{1}\right)$. Thus we have a unique direct sum decomposition $\mathfrak{C}_{V_{1}}^{\prime}=\mathfrak{C}_{1 V_{1}}^{\prime} \oplus \mathfrak{C}_{2 V_{1}}^{\prime}$ of $F$-crystals over $\operatorname{Spec}\left(V_{1}\right)$. Using this decomposition and the fact that both $F$-crystals $\mathfrak{C}_{1}^{\prime \prime}$ and $\mathfrak{C}_{2}^{\prime}$ over $\operatorname{Spec}\left(V_{2}\right)$ have ranks smaller than $r_{0}$, by induction we get the existence of $\tilde{l}_{C}$. This ends the induction. 
Let $\tilde{l}_{f}:=\max \left\{d\left(r_{0}, 0, c\right) \mid c \in S\left(0, h_{\mathfrak{C}}+\tilde{l}_{C}\right)\right\}$ with $d\left(r_{0}, 0, c\right)$ 's as in 2.4.1. The $h$-number of $\mathfrak{C}_{C}$ is at most $h_{\mathfrak{C}}+\tilde{l}_{C}$. So from 2.4.1 (applied over $k_{2}$ ) we get that there exists a DieudonnéFontaine $p$-divisible object $\mathfrak{C}_{0}^{\prime}$ over $\mathbf{F}_{p}$ for which we have an isogeny $i_{2}: \mathfrak{C}_{0 V_{1}}^{\prime} \rightarrow \mathfrak{C}_{C V_{1}}$ whose cokernel is annihilated by $p^{\tilde{l}_{f}}$. As the number of isomorphism classes of Dieudonné-Fontaine $p$-divisible objects over $\mathbf{F}_{p}$ that have $\mathcal{N}$ as their Newton polygons is finite, there exists a number $\tilde{l}_{\mathcal{N}} \in \mathbf{N}$ such that we have an isogeny $i_{3}: \mathfrak{C}_{0 V_{1}} \rightarrow \mathfrak{C}_{0 V_{1}}^{\prime}$ whose cokernel is annihilated by $p^{\tilde{l}_{\mathcal{N}}}$.

As $i_{V_{1}}$ we can take the composite isogeny $i_{1} \circ i_{2} \circ i_{3}$. Thus as $l$ we can take any integer greater than $\max \left\{h_{0} r_{0}^{2}, h_{\mathfrak{C}}, \tilde{l}_{C}+\tilde{l}_{f}+\tilde{l}_{\mathcal{N}}\right\}$.

\subsubsection{The open subscheme $U_{0}$}

With $l$ as in 6.1.2, let $m:=8 l+n+v+1$. We continue the proof of Theorem 6.1 by considering (see 2.8.2) the evaluation morphism

$$
\mathbf{E}\left(i_{V_{1}} ; W_{m+2 v}\left(V_{1}\right)\right): \mathbf{E}\left(\mathfrak{C}_{0} ; W_{m+2 v}\left(V_{1}\right)\right) \rightarrow \mathbf{E}\left(\mathfrak{C} ; W_{m+2 v}\left(V_{1}\right)\right)
$$

of $\mathcal{M}\left(W_{m+2 v}\left(V_{1}\right)\right)$. We apply 2.8.3(c) (with $q=m+2 v$ ) to this morphism. We get that there exist a finite field extension $k_{S, V}$ of $k_{S}$ and an open, affine subscheme $U_{\widetilde{V}}$ of the normalization of $U$ in $k_{S, V}$, such that $U_{\widetilde{V}}$ has a local ring $\widetilde{V}$ which is a discrete valuation ring and which dominates $V$ and moreover we have a morphism

$$
i_{U_{\tilde{V}}}(m+2 v): \mathbf{E}\left(\mathfrak{C}_{0} ; W_{m+2 v}\left(U_{\widetilde{V}}\right)\right) \rightarrow \mathbf{E}\left(\mathfrak{C} ; W_{m+2 v}\left(U_{\widetilde{V}}\right)\right)
$$

of $\mathcal{M}\left(W_{m+2 v}\left(U_{\widetilde{V}}\right)\right)$ whose cokernel is annihilated by $p^{l}$. See 2.1 for $i_{U_{\tilde{V}}}(m+v)$.

Let $\tilde{m} \in\{m, m+v\}$. Let $I_{\tilde{m}}$ be the set of morphisms $\mathfrak{C}_{0 k} / p^{\tilde{m}} \mathfrak{C}_{0 k} \rightarrow \mathfrak{C}_{k} / p^{\tilde{m}} \mathfrak{C}_{k}$ that are reductions mod $p^{\tilde{m}}$ of morphisms $\mathfrak{C}_{0 k} / p^{\tilde{m}+v} \mathfrak{C}_{0 k} \rightarrow \mathfrak{C}_{k} / p^{\tilde{m}+v} \mathfrak{C}_{k}$. Any morphism in $I_{\tilde{m}}$ lifts to a morphism $\mathfrak{C}_{0 k} \rightarrow \mathfrak{C}_{k}$, cf. 5.1.1(b), 5.1.2, and the definitions of $v$ and $n<m$; thus $I_{\tilde{m}}$ is a finite set. Let $J_{\tilde{m}}:=\left\{i \in I_{\tilde{m}} \mid p^{l} \operatorname{Coker}(i)=0\right\}$. Based on the case 2.8.3(a) of 2.8.3, by performing $(\mathcal{R} 1)$ we can assume that $J_{\tilde{m}}$ is the set of pullbacks of a set of morphisms $L_{\tilde{m}}$ of $\mathcal{M}\left(W_{\tilde{m}}\left(k_{S}\right)\right)$ whose cokernels are annihilated by $p^{l}$. The pullback of $i_{U_{\tilde{V}}}(m+v)$ to a morphism of $\mathcal{M}\left(W_{m+v}\left(k_{S, V}\right)\right)$ is also the pullback of a morphism in $L_{m+v}$. As $V=\widetilde{V} \cap k_{S}$, inside $W_{m+v}\left(k_{S, V}\right)$ we have $W_{m+v}(V)=W_{m+v}(\tilde{V}) \cap W_{m+v}\left(k_{S}\right)$. This implies that the pullback of $i_{U_{\tilde{V}}}(m+v)$ to a morphism of $\mathcal{M}\left(W_{m+v}(\tilde{V})\right)$ is in fact the pullback of a morphism of $\mathcal{M}\left(W_{m+v}(V)\right)$ whose cokernel is generically annihilated by $p^{l}$ (in the sense of 2.8.1). From the case 2.8.3(b) of 2.8.3 (applied with $\left(V_{1}, V\right)$ replaced by $(V, R)$ ), we get the existence of an open subscheme $U_{V}$ of $U$ that has $V$ as a local ring and such that we have a morphism

$$
i_{U_{V}}(m+v): \mathbf{E}\left(\mathfrak{C}_{0} ; W_{m+v}\left(U_{V}\right)\right) \rightarrow \mathbf{E}\left(\mathfrak{C} ; W_{m+v}\left(U_{V}\right)\right)
$$

of $\mathcal{M}\left(W_{m+v}\left(U_{V}\right)\right)$ whose cokernel is generically annihilated by $p^{l}$.

For $i \in J_{m}$ let $\mathcal{V}(i)$ be the set of all those discrete valuation rings $V$ of $U$ such that the pullback of $i_{U_{V}}(m)$ to a morphism of $\mathcal{M}\left(W_{m}(k)\right)$ is $i$. Let $U_{i}:=\bigcup_{V \in \mathcal{V}(i)} U_{V}$. Let

$$
i_{U_{i}}(m): \mathbf{E}\left(\mathfrak{C}_{0} ; W_{m}\left(U_{i}\right)\right) \rightarrow \mathbf{E}\left(\mathfrak{C} ; W_{m}\left(U_{i}\right)\right)
$$

be the morphism of $\mathcal{M}\left(W_{m}\left(U_{i}\right)\right)$ which is obtained by gluing together the morphisms $i_{U_{V}}(m)$ 's with $V \in \mathcal{V}(i)$. We have:

(a) if $U_{0}:=\bigcup_{i \in J_{m}} U_{i}$, then $U^{\text {top }} \backslash U_{0}^{\text {top }}$ has codimension at least 2 in $U^{\text {top }}$;

(b) for any $i \in J_{m}$, the cokernel of $i_{U_{i}}(m)$ is generically annihilated by $p^{l}$. 


\subsubsection{Gluing morphisms}

We now modify the morphisms $i_{U_{i}}(m)$ 's $\left(i \in J_{m}\right)$ so that they glue together to define a morphism

$$
i_{U_{0}}(m): \mathbf{E}\left(\mathfrak{C}_{0} ; W_{m}\left(U_{0}\right)\right) \rightarrow \mathbf{E}\left(\mathfrak{C} ; W_{m}\left(U_{0}\right)\right)
$$

of $\mathcal{M}\left(W_{m}\left(U_{0}\right)\right)$ whose cokernel is generically annihilated by $p^{3 l}$. If there exists $i \in J_{m}$ such that $U_{i}=U_{0}$, then $i_{U_{0}}(m)=i_{U_{i}}(m)$ has a cokernel generically annihilated by $p^{l}$.

We now assume that for all $i \in J_{m}$ we have $U_{0} \neq U_{i}$. The pullback of $i_{U_{i}}(m)$ to a morphism of $\mathcal{M}\left(W_{m}(k)\right)$ is $i \in J_{m}$. Let $f_{i}: \mathfrak{C}_{0 k} \rightarrow \mathfrak{C}_{k}$ be a morphism such that its reduction mod $p^{m}$ is $i$, cf. 5.1.1(b), 5.1.2, and the fact that $J_{m} \subseteq I_{m}$. As $m>l$ and $i \in J_{m}$, the cokernel of $f_{i}$ is annihilated by $p^{l}$ and so $f_{i}$ is an isogeny.

Let $f_{0} \in\left\{p^{l} f_{i} \mid i \in J_{m}\right\}$. We have:

(a) the image of $f_{0}$ lies inside the intersection of the images of all $f_{i}$ 's $\left(i \in J_{m}\right)$;

(b) the cokernel of $f_{0}$ is annihilated by $p^{2 l}$.

Let $s_{i}: \mathfrak{C}_{0 k} \rightarrow \mathfrak{C}_{0 k}$ be the isogeny such that we have $f_{0}=f_{i} \circ s_{i}$, cf. (a). So $\operatorname{Coker}\left(s_{i}\right)$ is annihilated by $p^{2 l}$, cf. (b). We know that $s_{i}$ is the pullback of an isogeny $\mathfrak{C}_{0 \mathbf{F}_{p} q_{0}} \rightarrow \mathfrak{C}_{0 \mathbf{F}_{p} q_{0}}$, cf. 2.2.3(b) applied to $\mathfrak{C}_{0}$. So as $\mathbf{F}_{p^{q_{0}}} \hookrightarrow R$, we get that the reduction mod $p^{m}$ of $s_{i}$ is the pullback of a (constant) morphism

$$
s_{U_{i}}(m): \mathbf{E}\left(\mathfrak{C}_{0} ; W_{m}\left(U_{i}\right)\right) \rightarrow \mathbf{E}\left(\mathfrak{C}_{0} ; W_{m}\left(U_{i}\right)\right)
$$

of $\mathcal{M}\left(W_{m}\left(U_{i}\right)\right)$ whose cokernel is annihilated by $p^{2 l}$. If $i_{1}, i_{2} \in J_{m}$, then the pullbacks of $i_{U_{i_{1}}}(m) \circ s_{U_{i_{1}}}(m)$ and $i_{U_{i_{2}}}(m) \circ s_{U_{i_{2}}}(m)$ to morphisms of $\mathcal{M}\left(W_{m}(k)\right)$ are the reduction of $f_{0} \bmod p^{m}$ and thus they coincide. This implies that the pullbacks of $i_{U_{i_{1}}}(m) \circ s_{U_{i_{1}}}(m)$ and $i_{U_{i_{2}}}(m) \circ s_{U_{i_{2}}}(m)$ to morphisms of $\mathcal{M}\left(W_{m}\left(U_{i_{1}} \cap U_{i_{2}}\right)\right)$ coincide. Thus the morphisms $i_{U_{i}}(m) \circ$ $s_{U_{i}}(m)$ indexed by $i \in J_{m}$ glue together to define a morphism $i_{U_{0}}(m): \mathbf{E}\left(\mathfrak{C}_{0} ; W_{m}\left(U_{0}\right)\right) \rightarrow$ $\mathbf{E}\left(\mathfrak{C} ; W_{m}\left(U_{0}\right)\right)$ of $\mathcal{M}\left(W_{m}\left(U_{0}\right)\right)$ whose cokernel is generically annihilated by $p^{3 l}=p^{l} p^{2 l}$, cf. 6.1.3(b) and the fact that $p^{2 l}$ annihilates $\operatorname{Coker}\left(s_{U_{i}}(m)\right)$.

It is easy to see that by performing $(\mathcal{R} 1)$ we can assume $p^{3 l}$ annihilates $\operatorname{Coker}\left(i_{U_{0}}(m)\right)$ but this will not be used in what follows.

6.1.5. LEMMA. - By performing $(\mathcal{R} 2)$, we can assume that $i_{U_{0}}(m)$ extends to a morphism $i_{S}(m): \mathbf{E}\left(\mathfrak{C}_{0} ; W_{m}(S)\right) \rightarrow \mathbf{E}\left(\mathfrak{C} ; W_{m}(S)\right)$ of $\mathcal{M}\left(W_{m}(S)\right)$.

Proof. - Let $S^{\prime}$ be the affine $S$-scheme of finite type that parametrizes morphisms between the two objects $\mathbf{E}\left(\mathfrak{C}_{0} ; W_{m}(S)\right)$ and $\mathbf{E}\left(\mathfrak{C} ; W_{m}(S)\right)$ of $\mathcal{M}\left(W_{m}(S)\right)$, cf. 2.8.4.1. Let $U_{0} \hookrightarrow S^{\prime}$ be the open embedding of $S$-schemes that defines $i_{U_{0}}(m)$. Let $U^{\prime}$ be the normalization of the Zariski closure of $U_{0}$ in $S^{\prime}$. As $S$ is an excellent scheme, the $S$-scheme $U^{\prime}$ is integral, normal, affine, and of finite type. As $U_{0}$ is an open subscheme of both $U$ and $U^{\prime}$ and due to 6.1.3(a), the affine morphism $U^{\prime} \times{ }_{S} U \rightarrow U$ between integral, normal, noetherian schemes is birational and has the property that any discrete valuation ring of $U$ is also a local ring of $U^{\prime} \times_{S} U$. Thus the morphism $U^{\prime} \times{ }_{S} U \rightarrow U$ is an isomorphism, cf. 2.9.1. So $U$ is an open subscheme of $U^{\prime}$. So by performing $(\mathcal{R} 2)$ (with $\widetilde{S}=U^{\prime}$ ), we can assume $U^{\prime}=S$. Thus we can speak about the morphism $i_{S}(m): \mathbf{E}\left(\mathfrak{C}_{0} ; W_{m}(S)\right) \rightarrow \mathbf{E}\left(\mathfrak{C} ; W_{m}(S)\right)$ of $\mathcal{M}\left(W_{m}(S)\right)$ that extends $i_{U_{0}}(m)$.

\subsubsection{Duals}

Let $\mathfrak{C}(l)$ be the Tate twist of $\mathfrak{C}$ by $(l)$, i.e. $\mathfrak{C}$ tensored with the pullback to $S$ of the $F$-crystal $\left(\mathbf{Z}_{p}, p^{l} 1 \mathbf{z}_{p}\right)$ over $\mathbf{F}_{p}$. As for modules, let $\mathfrak{C}^{*}$ be the dual of $\mathfrak{C}^{*}$ (one could call it a latticed $F$-isocrystal over $S$ ). We also define the Tate twist $\mathfrak{C}^{*}(l)$ of $\mathfrak{C}^{*}$ by $(l)$; it is an $F$-crystal over $S$ (as $l>h_{\mathfrak{C}}$ ). In a similar way we define $\mathfrak{C}_{0}^{*}(l)$. As $l>h_{0}, \mathfrak{C}_{0}^{*}(l)$ is a Dieudonné-Fontaine $p$-divisible object over $\mathbf{F}_{p}$ with non-negative slopes.

$4^{\text {e }}$ SÉRIE - TOME $39-2006-\mathrm{N}^{\circ} 2$ 
We repeat the constructions we performed for $\mathfrak{C}_{0}$ and $\mathfrak{C}$ (like the ones through which we got $l, U_{0}, i_{S}(m)$, etc.) in the context of $\mathfrak{C}_{0}^{*}(l)$ and $\mathfrak{C}^{*}(l)$. So by enlarging $l$ and by performing $(\mathcal{R} 1)$ and $(\mathcal{R} 2)$, we can assume there exists a morphism

$$
i_{S}^{*}(m): \mathbf{E}\left(\mathfrak{C}_{0}^{*}(l) ; W_{m}(S)\right) \rightarrow \mathbf{E}\left(\mathfrak{C}^{*}(l) ; W_{m}(S)\right)
$$

of $\mathcal{M}\left(W_{m}(S)\right)$ whose cokernel is generically annihilated by $p^{3 l}$ and which is the analogue of $i_{S}(m)$. As $l>h_{\mathfrak{C}}$, we think of $\mathbf{E}\left(\mathfrak{C}^{*}(l) ; W_{m}(S)\right)$ to be a "twisted dual" of $\mathbf{E}\left(\mathfrak{C} ; W_{m}(S)\right)$ in the sense that there exists a morphism

$$
j_{S}(m-l): \mathbf{E}\left(\mathfrak{C} ; W_{m-l}(S)\right) \rightarrow \mathbf{E}\left(\mathfrak{C}_{0} ; W_{m-l}(S)\right)
$$

which at the level of $\mathcal{O}_{W_{m-l}(S)}$-modules is the dual of $i_{S}^{*}(m-l)$. Thus the pullback of $j_{S}(m-l)$ to an object of $\mathcal{M}\left(W_{m-l}(k)\right)$ has a cokernel annihilated by $p^{3 l}$. By performing $\left(\mathcal{R}_{1}\right)$ we can assume $\operatorname{Coker}\left(j_{S}(m-l)\right)$ is generically annihilated by $p^{3 l}$, cf. 2.8.3(a).

\subsubsection{End of the proof of Theorem 6.1}

We will use the existence of the morphisms $i_{S}(m)$ and $i_{S}^{*}(m)$ to show that the assumption $U \neq S$ leads to a contradiction. Let $y: \operatorname{Spec}\left(k_{1}\right) \rightarrow S$ be a geometric point that does not factor through $U$. Let

$$
c_{S}(m-l):=j_{S}(m-l) \circ i_{S}(m-l): \mathbf{E}\left(\mathfrak{C}_{0} ; W_{m-l}(S)\right) \rightarrow \mathbf{E}\left(\mathfrak{C}_{0} ; W_{m-l}(S)\right) .
$$

We check that Coker $\left(c_{S}(m-l)\right)$ is annihilated by $p^{7 l}$. Let $c_{S}(m-l)_{\text {gen }}$ and $c_{S}(m-2 l)_{\text {gen }}$ be the morphisms of $\mathcal{M}\left(W_{m-l}(k)\right)$ and $\mathcal{M}\left(W_{m-2 l}(k)\right)$ (respectively) that are the natural pullbacks of $c_{S}(m-l)$ and $c_{S}(m-2 l)$ (respectively). As $c_{S}(m-2 l)_{\text {gen }}$ is the composite of two morphisms of $\mathcal{M}\left(W_{m-2 l}(k)\right)$ whose cokernels are annihilated by $p^{3 l}$, Coker $\left(c_{S}(m-2 l)_{\text {gen }}\right)$ is annihilated by $p^{6 l}$. As $c_{S}(m-2 l)_{\text {gen }}$ lifts to $c_{S}(m-l)_{\text {gen }}$ and as $l>h_{0} r_{0}^{2}, c_{S}(m-2 l)_{\text {gen }}$ is the pullback of a morphism of $\mathcal{M}\left(W_{m-2 l}\left(\mathbf{F}_{p^{q_{0}}}\right)\right)$ (cf. 2.2.3(a) applied with $(K, k)$ replaced by $\left.\left(k, \mathbf{F}_{p}\right)\right)$. So as $\mathbf{F}_{p^{q_{0}}} \hookrightarrow R$, we get that $\operatorname{Coker}\left(c_{S}(m-2 l)\right)$ itself is annihilated by $p^{6 l}$. Thus $p^{7 l}$ annihilates $\operatorname{Coker}\left(c_{S}(m-l)\right)$.

As the endomorphism $y^{*}\left(c_{S}(m-l)\right)=y^{*}\left(j_{S}(m-l)\right) \circ y^{*}\left(i_{S}(m-l)\right)$ of $\mathcal{M}\left(W_{m-l}(k)\right)$ has a cokernel annihilated by $p^{7 l}$, we get that $p^{7 l}$ annihilates $\operatorname{Coker}\left(y^{*}\left(i_{S}(m-l)\right)\right)$. Let $f_{y}: \mathfrak{C}_{0 k_{1}} \rightarrow y^{*}(\mathfrak{C})$ be a morphism that lifts $y^{*}\left(i_{S}(m-l-v)\right)$, cf. 5.1.1(b) and 5.1.2. As $m-v-l=7 l+n+1 \geqslant 7 l+1$ (cf. the definition of $m$ in 6.1.3) and as $p^{7 l}$ annihilates $\operatorname{Coker}\left(y^{*}\left(i_{S}(m-l-v)\right)\right)$ (by reasons of ranks) the morphism $f_{y}$ is injective and so an isogeny. So $y^{*}(\mathfrak{C})$ has Newton polygon $\mathcal{N}$. So $y$ factors through $U$. This contradicts the choice of $y$. Thus the existence of the morphisms $i_{S}(m)$ and $i_{S}^{*}(m)$ implies that $U=S$. As $U=S, U$ is affine. This ends the proof of Theorem 6.1.

\subsection{Proof of Main Theorem B}

We prove 1.6. Let $U$ be a reduced, locally closed subscheme of $S$ that is a stratum of $\mathcal{S}(\mathfrak{C})$. We have to show that $U$ is an affine $S$-scheme. It suffices to check this under the extra assumptions that $S=\operatorname{Spec}(R)$ is affine, that $U$ is an open, dense subscheme of $S$, and that the underlying $R$-module of $\mathbf{E}\left(\mathfrak{C} ; W_{1}(S)\right)$ is free. We will show that $U$ is an affine scheme. It suffices to check this under the extra assumption that $R$ is normal and perfect, cf. 2.9.2 applied with $X$ and $X^{\prime}$ replaced by $S$ and by the normalization of $\operatorname{Spec}\left(R^{\text {perf }}\right)$ (respectively).

Let $\mathcal{N}$ be the Newton polygon of pullbacks of $\mathfrak{C}_{U}$ via geometric points of $U$. Let $\mathfrak{C}_{0}, h_{0}, r_{0}, v$, and $n$ be associated to $\mathcal{N}$ and $h_{\mathfrak{C}}$ as in 5.4 (this makes sense even if $R$ is not an integral domain; 
see 2.1 for $\left.h_{\mathfrak{C}}\right)$. So $\mathfrak{C}_{0}=\left(M_{0}, \varphi_{0}\right)$ is a Dieudonné-Fontaine $p$-divisible object over $\mathbf{F}_{p}$ that has rank $r_{0}$, has $h$-number $h_{0}$, and has Newton polygon $\mathcal{N}$.

Let $\tilde{n}$ be the maximum between $2+h_{\mathfrak{C}}+2 \max \left\{d\left(r_{0}^{2}, s, c\right) \mid s \in S\left(0, h_{\mathfrak{C}}\right), c \in S\left(0,2 h_{\mathfrak{C}}\right)\right\}$ and $n$. If $(M, \varphi)$ is an $F$-crystal over an algebraically closed field $K$ of characteristic $p$ of rank $r_{0}$ and $h$-number at most $h_{\mathfrak{C}}$, then $(\operatorname{End}(M), \varphi)$ is a latticed $F$-isocrystal over $K$ whose rank is $r_{0}^{2}$, whose $s$-number is at most $h_{\mathfrak{C}}$, and whose $h$-number is at most $2 h_{\mathfrak{C}}$ (see end of 2.2.1(e)). Thus from 3.2.8(a) (applied with $G=\mathbf{G L}_{M}$ ) and 2.4.1 we get:

(i) any $F$-crystal over $K$ whose rank is $r_{0}$ and whose $h$-number is at most $h_{\mathfrak{C}}$, is uniquely determined up to isomorphism by its reduction $\bmod p^{\tilde{n}}$.

We consider quadruples of the form $(\tilde{k}, \widetilde{\Phi}, \widetilde{M}, \tilde{\varphi})$, where:

- $\tilde{k}$ is an algebraically closed field of characteristic $p$,

- $\widetilde{\Phi}$ is the Frobenius endomorphism of $W(\tilde{k})[[w]]$ that is compatible with $\sigma_{\tilde{k}}$ and that takes $w$ into $w^{p}$

- $\widetilde{M}$ is a free $W(\tilde{k})[[w]]$-module of rank $r_{0}$ equipped with a $\widetilde{\Phi}$-linear endomorphism $\tilde{\varphi}$,

which have the property that the Newton polygons and the $h$-numbers of extensions of $(\widetilde{M}, \tilde{\varphi})$ via $W(\tilde{k})$-homomorphisms $W(\tilde{k})[[w]] \rightarrow W(K)$ that are compatible with the Frobenius endomorphisms and that involve algebraically closed fields $K$ of characteristic $p$, are $\mathcal{N}$ and respectively are at most $h_{\mathfrak{C}}$.

We consider the unique $W(\tilde{k})$-monomorphism $W(\tilde{k})[[w]] \hookrightarrow W\left(\tilde{k}[[w]]^{\text {perf }}\right)$ that lifts the natural inclusion $\tilde{k}[[w]] \hookrightarrow \tilde{k}[[w]]^{\text {perf }}$ and that is compatible with the Frobenius endomorphisms $\widetilde{\Phi}$ and $\Phi_{\tilde{k}[[w]]_{\text {perf }}}$, cf. [22, p. 145]; it maps $w$ into $(w, 0,0, \ldots) \in W\left(\tilde{k}[[w]]^{\text {perf }}\right)$.

The results $[22,2.6 .1,2.6 .2,2.7 .1$, and 2.7.4] hold as well in the context of pairs of the form $(\widetilde{M}, \tilde{\varphi})$ that are not endowed with connections (one only has to disregard all details of loc. cit. that pertain to connections). So as in the proof of 6.1.2 we argue that there exists a number $l \in \mathbf{N}$ which has the properties that $l \geqslant \max \left\{h_{0} r_{0}^{2}, h_{\mathfrak{C}}\right\}$ and that for any quadruple $(\tilde{k}, \widetilde{\Phi}, \widetilde{M}, \tilde{\varphi})$ as above, there exists a monomorphism

$$
\left(M_{0} \otimes_{\mathbf{z}_{p}} W\left(\tilde{k}[[w]]^{\text {perf }}\right), \varphi_{0} \otimes \Phi_{\tilde{k}[[w]]^{\text {perf }}}\right) \hookrightarrow\left(\widetilde{M} \otimes_{W(\tilde{k})[[w]]} W\left(\tilde{k}[[w]]^{\text {perf }}\right), \tilde{\varphi} \otimes \Phi_{\tilde{k}[[w]]^{\text {perf }}}\right)
$$

whose cokernel is annihilated by $p^{l}$. Let $m:=8 l+\tilde{n}+v+1$ and $\tilde{m}:=m+2 v$.

Let $\left(O, \varphi_{O}\right):=\operatorname{proj} \lim _{t \in \mathbf{N}} \mathbf{E}\left(\mathfrak{C} ; W_{t}(S)\right)$. As the underlying $R$-module of the object $\mathbf{E}\left(\mathfrak{C} ; W_{1}(S)\right)$ is free, $O$ is a free $W(R)$-module of rank $r_{0}$. Moreover $\varphi_{O}$ is a $\Phi_{R}$-linear endomorphism of $O$. As $R$ is perfect, the $W_{t}(R)$-module of differentials $\Omega_{W_{t}(R)}$ is trivial. So the connection on $O$ induced by $\mathfrak{C}$ is trivial. From this and [22, p. 145] we get that the pair $\left(O, \varphi_{O}\right)$ determines $\mathfrak{C}$ up to isomorphism. Let $\mathcal{B}=\left\{e_{1}, \ldots, e_{r_{0}}\right\}$ be a $W(R)$-basis of $O$. Let $B \in M_{r_{0} \times r_{0}}(W(R))$ be the matrix representation of $\varphi_{O}$ with respect to $\mathcal{B}$. Let $B_{\tilde{m}} \in M_{r_{0} \times r_{0}}\left(W_{\tilde{m}}(R)\right)$ be $B \bmod p^{\tilde{m}}$. Let $R^{0}$ be a finitely generated $\mathbf{F}_{p^{-}}$-subalgebra of $R$ which contains the components of the Witt vectors of length $\tilde{m}$ with coefficients in $R$ that are entries of $B_{\tilde{m}}$; so $B_{\tilde{m}} \in M_{r_{0} \times r_{0}}\left(W_{\tilde{m}}\left(R^{0}\right)\right)$. Let $S^{0}:=\operatorname{Spec}\left(R^{0}\right)$.

Let $\varphi_{O}^{\prime}$ be a $\Phi_{R}$-linear endomorphism of $O$ whose matrix representation with respect to $\mathcal{B}$ is a matrix $B^{\prime} \in M_{r_{0} \times r_{0}}\left(W\left(R^{0}\right)\right) \subseteq M_{r_{0} \times r_{0}}(W(R))$ that lifts $B_{\tilde{m}}$. Let $\mathfrak{C}_{S}^{\prime}$ be the $F$-crystal over $S$ that corresponds to the pair $\left(O, \varphi_{O}^{\prime}\right)$, cf. the above part that refers to [22, p. 145]. As $B^{\prime} \in M_{r_{0} \times r_{0}}\left(W\left(R^{0}\right)\right)$, loc. cit. also implies that $\mathfrak{C}_{S}^{\prime}$ is the pullback to $S$ of an $F$-crystal $\mathfrak{C}^{\prime}$ over $\operatorname{Spec}\left(R^{0 \text { perf }}\right)$. As $B$ and $B^{\prime}$ are congruent $\bmod p^{\tilde{m}}$, we can identify $\mathfrak{C} / p^{\tilde{m}} \mathfrak{C}^{\mathfrak{C}}$ with $\mathfrak{C}_{S}^{\prime} / p^{\tilde{m}} \mathfrak{C}_{S}^{\prime}$. It is easy to see that due to this identification and to (i), the two Newton polygon stratifications $\mathcal{S}(\mathfrak{C})$ and $\mathcal{S}\left(\mathfrak{C}_{S}^{\prime \prime}\right)$ of $S$ coincide. Thus to prove that $U$ is affine we can assume that $B=B^{\prime}$, $R=R^{0 \text { perf }}$, and $\mathfrak{C}=\mathfrak{C}^{\prime}$. As $R=R^{0 \text { perf }}$, there exists a unique open subscheme $U^{0}$ of $S^{0}$ such that we have $U=U^{0} \times S^{0} S$.

4e SÉRIE - TOME $39-2006-\mathrm{N}^{\circ} 2$ 
To prove that $U$ is affine, it suffices to show that $U^{0}$ is affine. The scheme $U^{0}$ is affine if and only if its intersection with any irreducible component $C^{0}$ of $S^{0}$ is affine, cf. [16, Ch. II, Cor. (6.7.3)]. Thus by replacing $\left(S^{0}, S\right)$ with $\left(C^{0}, C^{0 \text { perf }}\right)$, we can assume that both $S^{0}$ and $S$ are integral schemes. By replacing $S^{0}$ and $S$ with their normalizations (cf. 2.9.2), we can also assume that $S^{0}$ is a normal $\operatorname{Spec}\left(\mathbf{F}_{p}\right)$-scheme of finite type.

As $B_{\tilde{m}} \in M_{r_{0} \times r_{0}}\left(W_{\tilde{m}}\left(R^{0}\right)\right)$, for any $j \in S(1, \tilde{m})$ and for every $S^{0}$-scheme $S_{1}$ we can speak about the object $\mathbf{E}\left(\mathfrak{C} ; W_{j}\left(S_{1}\right)\right)$ of $\mathcal{M}\left(W_{j}\left(S_{1}\right)\right)$ whose underlying $\mathcal{O}_{W_{j}\left(S_{1}\right)}$-module is the free $\mathcal{O}_{W_{j}\left(S_{1}\right)}$-module that has $\mathcal{B}$ as an $\mathcal{O}_{W_{j}\left(S_{1}\right)}$-basis and whose underlying Frobenius endomorphism has a matrix representation with respect to $\mathcal{B}$ which is the natural image of $B_{\tilde{m}}$ in $M_{r_{0} \times r_{0}}\left(\mathcal{O}_{W_{j}\left(S_{1}\right)}\right)$. If $S_{1}$ is an $S$-scheme, then $\mathbf{E}\left(\mathfrak{C} ; W_{j}\left(S_{1}\right)\right)$ is precisely the object of $\mathcal{M}\left(W_{j}\left(S_{1}\right)\right)$ defined in 2.8.2.

Let $V^{0}$ be an arbitrary local ring of $U^{0}$ that is a discrete valuation ring. Let $w_{0}$ be a uniformizer of it. Let $V_{3}^{0}$ be a $V^{0}$-algebra that is a complete discrete valuation ring, that has $w_{0}$ as a uniformizer, and that has an algebraically closed residue field $k_{3}$. So $V_{3}^{0}$ is isomorphic to $k_{3}\left[\left[w_{0}\right]\right]$, with $w_{0}$ viewed as a variable. We identify $w_{0}^{p^{-\tilde{m}}}$ with a uniformizer of $V_{2}^{0}:=V_{3}^{0\left(p^{\tilde{m}}\right)}$. So $k_{2}:=k_{3}^{\left(p^{\tilde{m}}\right)}$ is the residue field of $V_{2}^{0}$. Let $V_{1}^{0}:=k_{2}\left[\left[w_{0}^{p^{-\tilde{m}}}\right]\right]^{\text {perf }}=V_{2}^{0 \text { perf }}$. For $j \in S(1, \tilde{m})$ let $W_{j}^{0}:=W\left(k_{2}\right)\left[\left[w_{0}^{p^{-j}}\right]\right]$ be endowed with the Frobenius endomorphism $\Phi_{W_{j}^{0}}$ that is compatible with $\sigma_{k_{2}}$ and that takes $w_{0}^{p^{-j}}$ into $w_{0}^{p^{-j+1}}$. Let $f_{j}^{0}: W_{j}^{0} \hookrightarrow W\left(k_{2}\left[\left[w_{0}^{p^{-j}}\right]\right]\right)$ be the $W\left(k_{2}\right)$ monomorphism that lifts the canonical identification $k_{2}\left[\left[w_{0}^{p^{-j}}\right]\right]=W_{1}\left(k_{2}\left[\left[w_{0}^{p^{-j}}\right]\right]\right)$ and that takes $w_{0}^{p^{-j}}$ into the Witt vector $\left(w_{0}^{p^{-j}}, 0,0, \ldots\right) \in W\left(k_{2}\left[\left[w_{0}^{p^{-j}}\right]\right]\right)$. The following two properties hold:

(ii) each $f_{j}^{0}$ is compatible with Frobenius endomorphisms, and

(iii) if $j<\tilde{m}$, the restriction of $f_{j+1}^{0}$ to the $W\left(k_{0}\right)$-subalgebra $W_{j}^{0}$ of $W_{j+1}^{0}$ is $f_{j}^{0}$.

We recall that if $x=\left(x_{0}, x_{1}, \ldots, x_{\tilde{m}}\right)$ is a Witt vector of length $\tilde{m}$, then $p x=$ $\left(0, x_{0}^{p}, x_{1}^{p}, \ldots, x_{\tilde{m}-1}^{p}\right)$. Based on this and (ii) and (iii), by induction on $j \in S(1, \tilde{m})$ we get that the image (via the natural monomorphism $R^{0} \hookrightarrow k_{2}\left[\left[w_{0}^{p^{-j}}\right]\right]$ ) of the matrix $B=B^{\prime} \in$ $M_{r_{0} \times r_{0}}\left(W\left(R^{0}\right)\right)$ in $M_{r_{0} \times r_{0}}\left(W_{j}\left(k_{2}\left[\left[w_{0}^{p^{-j}}\right]\right]\right)\right)$, belongs to $M_{r_{0} \times r_{0}}\left(W_{j}^{0} / p^{j} W_{j}^{0}\right)$. Thus the image of $B=B^{\prime}$ in $M_{r_{0} \times r_{0}}\left(W_{\tilde{m}}\left(k_{2}\left[\left[w_{0}^{p^{-\tilde{m}}}\right]\right]\right)\right)$, belongs to $M_{r_{0} \times r_{0}}\left(W_{\tilde{m}}^{0} / p^{\tilde{m}} W_{\tilde{m}}^{0}\right)$ and so it lifts to a matrix $\widetilde{B} \in M_{r_{0} \times r_{0}}\left(W_{\tilde{m}}^{0}\right)$.

Let $\widetilde{M}:=\bigoplus_{i=1}^{r_{0}} W_{\tilde{m}}^{0} e_{i}$. Let $\tilde{\varphi}$ be the $\Phi_{W_{\tilde{m}}^{0}}$-linear endomorphism of $\widetilde{M}$ whose matrix representation with respect to $\mathcal{B}$ is $\widetilde{B}$. The extension of $(\widetilde{M}, \tilde{\varphi})$ via a $W\left(k_{2}\right)$-homomorphism $W_{\tilde{m}}^{0} \rightarrow W(K)$ compatible with Frobenius endomorphisms, has the $h$-number at most $h_{\mathfrak{C}}$ (as $\tilde{m}>h_{\mathfrak{C}}$ ) and has Newton polygon $\mathcal{N}$ (cf. (i) and the fact that $V^{0}$ is a local ring of $U^{0}$ ). So there exists a monomorphism

$$
\left(M_{0} \otimes_{\mathbf{z}_{p}} W\left(V_{1}^{0}\right), \varphi_{0} \otimes \Phi_{V_{1}^{0}}\right) \hookrightarrow\left(\widetilde{M} \otimes_{W_{\tilde{m}}^{0}} W\left(V_{1}^{0}\right), \tilde{\varphi} \otimes \Phi_{V_{1}^{0}}\right)
$$

whose cokernel is annihilated by $p^{l}$, cf. the choice of $l$ (applied with $k_{2}\left[\left[w_{0}^{p^{-\tilde{m}}}\right]\right]$ instead of $\tilde{k}[[w]])$. Thus there exists a morphism $\mathbf{E}\left(\mathfrak{C}_{0} ; W_{\tilde{m}}\left(V_{1}^{0}\right)\right) \rightarrow \mathbf{E}\left(\mathfrak{C} ; W_{\tilde{m}}\left(V_{1}^{0}\right)\right)$ of $\mathcal{M}\left(W_{\tilde{m}}\left(V_{1}^{0}\right)\right)$ whose cokernel is annihilated by $p^{l}$.

As in Sections 6.1.3 to 6.1.7 we only used evaluation functors $\mathbf{E}$ and pullbacks of $F$-crystals over $S$ via geometric points of $S$ and as for any algebraically closed field $K$ the map $S(K) \rightarrow$ $S^{0}(K)$ is bijective, the rest of the proof that $U^{0}$ is affine is the same as in Sections 6.1.3 to 6.1.7 (but with the role of $(n, m)$ being replaced with the one of $(\tilde{n}, \tilde{m}))$. We will only add two extra sentences.

From 2.8.3(c) (applied with $q$ replaced by $\tilde{m}$ ) we get that there exist: 
(iv) an open subscheme $U_{\widetilde{V}^{0}}^{0}$ of the normalization of $U^{0}$ in a finite field extension $k_{S^{0}, V^{0}}$ of the field of fractions $k_{S^{0}}$ of $S^{0}$, a local ring $\widetilde{V}^{0}$ of $U_{\widetilde{V}^{0}}^{0}$ which dominates $V^{0}$, and a morphism $i_{U_{\tilde{V}^{0}}^{0}}(\tilde{m}): \mathbf{E}\left(\mathfrak{C}_{0} ; W_{\tilde{m}}\left(U_{\widetilde{V}^{0}}^{0}\right)\right) \rightarrow \mathbf{E}\left(\mathfrak{C} ; W_{\tilde{m}}\left(U_{\widetilde{V}^{0}}^{0}\right)\right)$ of $\mathcal{M}\left(W_{\tilde{m}}\left(U_{\widetilde{V}^{0}}^{0}\right)\right)$ whose cokernel is annihilated by $p^{l}$.

In connection with the last two paragraphs of 6.1.3 and with Sections 6.1.4 to 6.1.7, we only have to add an upper right index 0 to all schemes that (modulo the two operations of 6.1.1) are about to be introduced; thus we get open subschemes $U_{V^{0}}^{0}, U_{i}^{0}$, and $U_{0}^{0}$ of $U^{0}$, etc. This ends the proof of 1.6 .

6.3. Remarks. - (a) Let $S$ be an integral, locally noetherian scheme. Let $\mathfrak{C}$ and $\mathcal{S}(\mathfrak{C})$ be as in the beginning of Section 6 . Let $U$ be the unique stratum of $\mathcal{S}(\mathfrak{C})$ which is an open subscheme of $S$. The open embedding $U \hookrightarrow S$ is an affine morphism, cf. 6.1. This implies that either $U=S$ or $S^{\text {top }} \backslash U^{\text {top }}$ is of pure codimension $1 \mathrm{in} S^{\text {top }}$. It suffices to check this statement under the extra assumptions that (to be compared with the first paragraph of the proof of 6.1) $S$ is also local, complete, and normal and that $S^{\text {top }} \backslash U^{\text {top }}$ has pure codimension $c \in \mathbf{N}$ in $S^{\text {top }}$. If $c>1$, then by applying 2.9.1 to the affine, birational open embedding $U \hookrightarrow S$ we get that $U=S$. Thus $c$ must be 1 .

Thus 6.1 implies the following result of de Jong and Oort (see [10, 4.1]): if $S$ is a local, integral, noetherian ring and if $U$ contains the complement in $S$ of the closed point of $S$, then either the dimension of $S$ is at most 1 or $S=U$. The converse of this implication holds, provided our scheme $S$ is locally factorial. But in general the result of de Jong and Oort does not imply 6.1. This is so as there exist integral, normal, noetherian, affine schemes $S=\operatorname{Spec}(R)$ that have a prime Weil divisor $C$ such that the open subscheme $S \backslash C$ of $S$ is not an affine scheme. Here is one classical example.

Let $R:=k\left[x_{1}, x_{2}, x_{3}, x_{4}\right] /\left(x_{1} x_{4}-x_{2} x_{3}\right)$. Let $C:=\operatorname{Spec}\left(k\left[x_{1}, x_{3}\right]\right)$ be the irreducible divisor of $S$ defined by the equations $x_{4}=x_{2}=0$. The open subscheme $S \backslash C$ of $S$ is the union of $\operatorname{Spec}\left(R\left[\frac{1}{x_{2}}\right]\right)$ and $\operatorname{Spec}\left(R\left[\frac{1}{x_{4}}\right]\right)$ and thus its $R$-algebra of global functions is $R\left[\frac{1}{x_{2}}\right] \cap R\left[\frac{1}{x_{4}}\right]$. But $W:=\operatorname{Spec}\left(R\left[\frac{1}{x_{2}}\right] \cap R\left[\frac{1}{x_{4}}\right]\right)$ is an affine $S$-scheme whose fibre over the point of $S$ defined by $x_{1}=x_{2}=x_{3}=x_{4}=0$ is non-empty. Thus the natural morphism $S \backslash C \rightarrow W$ is not an isomorphism and so the scheme $S \backslash C$ is not affine.

(b) Let $(M, \varphi)$ be an $F$-crystal over a perfect field $k$ of characteristic $p$. It is easy to see that $\left[22,1.4\right.$ and 1.5] implies the existence of a number $n_{0} \in \mathbf{N}$ such that for any $g \in \mathbf{G L}_{M}(W(k))$ the Newton polygon of $(M, g \varphi)$ depends only on $g \bmod p^{n_{0}}$. For instance, if $k=\bar{k}$ we can take $n_{0}$ to be the number $n_{\text {fam }}$ of 3.1 .5 for $G=\mathbf{G L}_{M}$. One can use this (in a way similar to the first part of 6.2) to define Newton polygon stratifications for reductions modulo adequate powers of $p$ of $F$-crystals over reduced $\operatorname{Spec}\left(\mathbf{F}_{p}\right)$-schemes.

For instance, it can be easily checked starting from 1.3 and $[21,4.4(\mathrm{e})]$ that any truncated Barsotti-Tate group $\mathcal{G}_{S}$ of level $T(r, d)$ over a reduced $\operatorname{Spec}\left(\mathbf{F}_{p}\right)$-scheme $S$ which has height $r$ and relative dimension $d$, defines a stratification $\mathcal{S}\left(\mathcal{G}_{S}\right)$ of $S$ as follows. The association $\mathcal{G}_{S} \rightarrow$ $\mathcal{S}\left(\mathcal{G}_{S}\right)$ is uniquely determined by the following two properties:

(i) it is functorial with respect to pullbacks, and

(ii) if there exists a $p$-divisible group $\mathcal{D}_{S}$ over $S$ such that $\mathcal{D}_{S}\left[p^{T(r, d)}\right]$ is isomorphic to $\mathcal{G}_{S}$, then $\mathcal{S}\left(\mathcal{G}_{S}\right)$ is the Newton polygon stratification of $S$ defined by the $F$-crystal over $S$ that is associated naturally to $\mathcal{D}_{S}$.

$4^{\mathrm{e}}$ SÉRIE - TOME $39-2006-\mathrm{N}^{\circ} 2$ 


\section{Acknowledgements}

We would like to thank the University of Utah and the University of Arizona for the good conditions provided during the writing of this paper and D. Ulmer, G. Faltings and the referee for valuable comments.

\section{REFERENCES}

[1] Borel A., Linear Algebraic Groups, Graduate Texts in Math., vol. 126, Springer, Berlin, 1991.

[2] Bosch S., LÜtkebohmert W., Raynaud M., Néron Models, Ergeb. Math. Grenzgeb. (3), vol. 21, Springer, Berlin, 1990.

[3] Conway J.H., Curtis R.T., Norton S.P., Parker R.A., Wilson R.A., Atlas of Finite Groups, Oxford University Press, Eynsham, 1985, xxxiv+252 pp.

[4] Cremona J.E., Algorithms for Modular Elliptic Curves, Cambridge University Press, Cambridge, 1992.

[5] Demazure M., Lectures on $p$-Divisible Groups, Lecture Notes in Math., vol. 302, Springer, Berlin, 1972.

[6] Demazure M., Grothendieck A., et al., Schémas en groupes, vols. I-III, Lecture Notes in Math., vols. 151-153, Springer, Berlin, 1970.

[7] DiEudonné J., Groupes de Lie et hyperalgèbres de Lie sur un corps de caractérisque $p>0$ (IV), Amer. J. Math. 77 (1955) 429-452.

[8] DE JONG J., Finite locally free group schemes in characteristic $p$ and Dieudonné modules, Invent. Math. 114 (1) (1993) 89-137.

[9] DE Jong J., Crystalline Dieudonné module theory via formal and rigid geometry, Inst. Hautes Études Sci. Publ. Math. 82 (1995) 5-96.

[10] De Jong J., OORT F., Purity of the stratification by Newton polygons, J. Amer. Math. Soc. 13 (1) (2000) 209-241.

[11] Faltings G., Crystalline cohomology and p-adic Galois representations, in: Algebraic Analysis, Geometry, and Number Theory, Baltimore, MD, 1988, Johns Hopkins University Press, Baltimore, MD, 1989, pp. 25-80.

[12] Faltings G., Integral crystalline cohomology over very ramified valuation rings, J. Amer. Math. Soc. 12 (1) (1999) 117-144.

[13] Faltings G., Chai C.-L., Degeneration of Abelian Varieties, Ergeb. Math. Grenzgeb. (3), vol. 22, Springer, Berlin, 1990.

[14] Fontaine J.-M., Groupes p-divisibles sur les corps locaux, J. Astérisque, vol. 47/48, Soc. Math. de France, Paris, 1977.

[15] Fontaine J.-M., Laffaille G., Construction de représentations p-adiques, Ann. Sci. École Norm. Sup. 15 (4) (1982) 547-608.

[16] GrothendiEcK A., Éléments de géométrie algébrique. II. Étude globale élémentaire de quelques classes de morphisms, Inst. Hautes Études Sci. Publ. Math., vol. 11, 1961.

[17] Grothendieck A., Éléments de géométrie algébrique. III. Étude cohomologique des faisceaux cohérents, Première partie, Inst. Hautes Études Sci. Publ. Math., vol. 11, 1963.

[18] Grothendieck A., Éléments de géométrie algébrique. IV. Étude locale des schémas et des morphismes de schéma, Inst. Hautes Études Sci. Publ. Math., vol. 20, 1964, vol. 24, 1965, vol. 28, 1996, and vol. 32, 1967.

[19] Grothendieck A., Groupes de Barsotti-Tate et cristaux de Dieudonné, Sém. Math. Sup., vol. 45, (1970), Les presses de l'Université de Montréal, Montréal, Quebec, 1974.

[20] Helgason S., Differential Geometry, Lie Groups, and Symmetric Spaces, Academic Press, New York, 1978.

[21] Illusie L., Déformations des groupes de Barsotti-Tate (d'après A. Grothendieck), in: Seminar on Arithmetic Bundles: The Mordell Conjecture, Paris, 1983/84, in: J. Astérisque, vol. 127, Soc. Math. de France, Paris, 1985, pp. 151-198. 
[22] KATZ N., Slope filtration of F-crystals, in: Journées de Géométrie Algébrique de Rennes, Rennes, 1978, vol. I, in: J. Astérisque, vol. 63, Soc. Math. de France, Paris, 1979, pp. 113-163.

[23] Katz N., Serre-Tate local moduli, in: Algebraic Surfaces Orsay, 1976-1978, in: Lecture Notes in Math., vol. 868, Springer, Berlin, 1981, pp. 138-202.

[24] Kotтwitz R.E., Isocrystals with additional structure, Comp. Math. 56 (2) (1985) 201-220.

[25] Kotтwitz R.E., Points on some Shimura varieties over finite fields, J. Amer. Math. Soc. 5 (2) (1992) 373-444.

[26] KRAFT, H., Kommutative algebraische $p$-Gruppen (mit Anwendungen auf $p$-divisible Gruppen und abelsche Varietäten), Manuscript, Univ. Bonn, 1975, 86 p.

[27] Li K.-Z., OORT F., Moduli of Supersingular Abelian Varieties, Lecture Notes in Math., vol. 1680, Springer, Berlin, 1998.

[28] Manin J.I., The theory of formal commutative groups in finite characteristic, Russian Math. Surveys 18 (6) (1963) 1-83.

[29] Matsumura H., Commutative Algebra, second ed., Benjamin/Cummings, Reading, MA, 1980.

[30] Messing W., The Crystals Associated to Barsotti-Tate Groups, with Applications to Abelian Schemes, Lecture Notes in Math., vol. 264, Springer, Berlin, 1972.

[31] Milne J.S., The points on a Shimura variety modulo a prime of good reduction, in: The Zeta Functions of Picard Modular Surfaces, Univ. Montréal Press, Montréal, Quebec, 1992, pp. 153255.

[32] Mumford D., Abelian Varieties, Tata Inst. of Fund. Research Studies in Math., vol. 5, Tata Institute of Fundamental Research, Oxford University Press, Bombay, London, 1970, viii+242 pp. (Reprinted 1988).

[33] Mumford D., Fogarty J., Kirwan F., Geometric Invariant Theory, third ed., Ergeb. Math. Grenzgeb. (2), vol. 34, Springer, Berlin, 1994.

[34] OORT F., A stratification of a moduli space of abelian varieties, in: Moduli of Abelian Varieties, Texel Island, 1999, in: Progr. Math., vol. 195, Birkhäuser, Basel, 2001, pp. 345-416.

[35] OORT F., Newton polygon strata in the moduli space of abelian varieties, in: Moduli of Abelian Varieties, Texel Island, 1999, in: Progr. Math., vol. 195, Birkhäuser, Basel, 2001, pp. 417-440.

[36] OORT F., Foliations in moduli spaces of abelian varieties, J. Amer. Math. Soc. 17 (2) (2004) 267-296.

[37] RApOpORT M., Richartz M., On the classification and specialization of $F$-isocrystals with additional structure, Comp. Math. 103 (2) (1996) 153-181.

[38] SerRe J.-P., Galois Cohomology, Springer, Berlin, 1997.

[39] TATE J., Classes d'isogénie des variétés sur un corps fini (d'après J. Honda), in: Sém. Bourbaki 1968/69, Exp. 352, in: Lecture Notes in Math., vol. 179, Springer, Berlin, 1971, pp. 95-110.

[40] VASIU A., Integral canonical models of Shimura varieties of preabelian type, Asian J. Math. 3 (2) (1999) 401-518.

[41] ZINK T., On the slope filtration, Duke Math. J. 109 (1) (2001) 79-95.

[42] Wintenberger J.-P., Un scindage de la filtration de Hodge pour certaines varietés algebriques sur les corps locaux, Ann. of Math. (2) 119 (3) (1984) 511-548.

(Manuscrit reçu le 11 juin 2004; accepté, après révision, le 16 décembre 2005.)

\footnotetext{
Adrian VASIU

University of Arizona,

Department of Mathematics,

617 N. Santa Rita, PO Box 210089,

Tucson, AZ 85721-0089, USA

E-mail: adrian@math.arizona.edu
}

$4^{\text {e }}$ SÉRIE - TOME $39-2006-\mathrm{N}^{\circ} 2$ 